\title{
A Unified Spectro-Geometric-Ritz Method for Vibration Analysis of Open and Closed Shells with Arbitrary Boundary Conditions
}

\author{
Dongyan Shi, ${ }^{1}$ Yunke Zhao, ${ }^{1}$ Qingshan Wang, ${ }^{1}$ Xiaoyan Teng,, and Fuzhen Pang ${ }^{2}$ \\ ${ }^{1}$ College of Mechanical and Electrical Engineering, Harbin Engineering University, Harbin 150001, China \\ ${ }^{2}$ College of Shipbuilding Engineering, Harbin Engineering University, Harbin 150001, China \\ Correspondence should be addressed to Qingshan Wang; wangqingshanxlz@hotmail.com
}

Received 18 September 2015; Accepted 16 December 2015

Academic Editor: Tai Thai

Copyright (c) 2016 Dongyan Shi et al. This is an open access article distributed under the Creative Commons Attribution License, which permits unrestricted use, distribution, and reproduction in any medium, provided the original work is properly cited.

\begin{abstract}
This paper presents free vibration analysis of open and closed shells with arbitrary boundary conditions using a spectro-geometricRitz method. In this method, regardless of the boundary conditions, each of the displacement components of open and closed shells is represented simultaneously as a standard Fourier cosine series and several auxiliary functions. The auxiliary functions are introduced to accelerate the convergence of the series expansion and eliminate all the relevant discontinuities with the displacement and its derivatives at the boundaries. The boundary conditions are modeled using the spring stiffness technique. All the expansion coefficients are treated equally and independently as the generalized coordinates and determined using Rayleigh-Ritz method. By using this method, a unified vibration analysis model for the open and closed shells with arbitrary boundary conditions can be established without the need of changing either the equations of motion or the expression of the displacement components. The reliability and accuracy of the proposed method are validated with the FEM results and those from the literature.
\end{abstract}

\section{Introduction}

Open and closed shells, consisting of segments of shells of revolution (e.g., cylindrical, conical, and spherical shells), are important structural elements commonly used in many engineering fields such as aerospace crafts, naval vehicles, and construction buildings, owing to their excellent mechanical and physical properties. It is noticed that the open and closed shells in these applications often work in complex environmental conditions and can be subjected to a variety of boundary conditions including all the classical cases, elastic restraints, point supports, and their combinations as well as suffering various dynamic loads, which may result in violent vibrations and structural collapse. Furthermore, the shells used in the practical engineering may have different geometries based mainly on their curvature characteristics. For the sake of presenting the proposed solution clearly, the open and closed shells considered in the present work are focused on cylindrical, conical, and spherical shells, which are most frequently used in the engineering application.

The development of accurate shell theories has been the subjected of significant research interest for many years, and a large number of shell theories based on different approximations and assumptions have been proposed. A careful selection of the appropriate shell theory is crucial for a feasible vibration analysis of practical shells. It has been reported more than a century ago by Lord Rayleigh and A.E.H. Love. And, thenceforth, the wide use of these structures has motivated a huge amount of research efforts in developing more accurate, efficient, and applicable theories and modeling techniques, aiming to provide insight into vibration behaviors and sound radiations and reliable design of these shells. Survey articles and monographs oriented to such contributions may be found in Carrera [1], Liew et al. [2], Qatu et al. [3], Leissa [4], and Reddy [5]. Classical shell theories were developed originally for thin shells based on the Kirchhoff-Love kinematic hypothesis that the straight lines normal to the unreformed reference surface remain straight and normal to the reference surface after deformation. This hypothesis is equivalent to the disregard of the effect of transverse shear deformations of shells.

So far, some of the static and dynamic behaviors of the closed shells with classical boundary conditions had been 
presented precisely by researchers. Starting from Flügge's three equations of motion for a uniform thin cylindrical shell, Warburton [6] gave a general solution, from which the dependence of natural frequencies on shell dimensions and mode number can be investigated for any end conditions. Bert and Malik [7] made a step forward in furthering the potential of the DQM in the area of structural mechanics by introducing the application of the differential quadrature method (DQM) to the dynamic analysis of thin circular cylindrical shells. Lam and Loy [8] employed a formulation based on Love's first approximation theory and with beam functions used as axial modal functions in the Ritz procedure to study the effects of boundary conditions on the free vibration characteristics for a multilayered cylindrical shell. Loy and Lam [9] presented an approximate analysis using a layer-wise approach to study the vibration of thick circular cylindrical shells on the basis of three-dimensional theory of elasticity. Utilizing the infinite circular cylinders solution based on the technique of variables separation, Mofakhami et al. [10] developed a general solution to analyze the vibration of finite circular cylinders. Dai et al. [11] obtained an exact series solution for the vibration analysis of circular cylindrical shells with arbitrary boundary conditions using the elastic equations based on Flügge's theory. In this series solution, each of the three displacements was represented by a Fourier series and auxiliary functions and sought in a strong form by letting the solution exactly satisfy both the governing differential equations and the boundary conditions on a point-wise basis. Chen et al. [12] developed an exact solution to obtain the free vibrations of cylindrical shell with nonuniform elastic boundary constraints using an improved Fourier series method. Khalili et al. [13] presented a closed-form formulation of three-dimensional (3D) refined higher-order shear deformation theory (RHOST) for the free vibration analysis of simply supported-simply supported and clamped-clamped homogenous isotropic circular cylindrical shells. Jin et al. [14] developed a unified and exact solution method for the free vibration analysis of composite laminated cylindrical shells with various boundary condition and arbitrary lamination schemes. Viola and Tornabene [15] used the Generalized Differential Quadrature (GDQ) method to study the free vibration of conical shell structures. Liew et al. $[16,17]$ carried out the free vibration analysis of thin conical shells under different boundary conditions using the element-free $k p$-Ritz method. Shu [18] applied the global method of Generalized Differential Quadrature (GDQ) for the first time to study the free vibration of isotropic conical shells. Tornabene et al. [19, 20] focused on the dynamic behavior of moderately thick functionally graded conical, cylindrical shells and annular plates based on the first-order shear deformation theory (FSDT). Wu and Lee [21] presented the free vibration analysis of laminated conical shells with variable stiffness using the method of differential quadrature (DQ). Tornabene and Viola [22] used the technique known as the Generalized Differential Quadrature (GDQ) to deal with the dynamical behavior of hemispherical domes and spherical shell panels. Lee [23] applied the pseudospectral method to the axisymmetric and asymmetric free vibration analysis of spherical caps, in which the displacements and the rotations were expressed by Chebyshev polynomials and Fourier series. Gautham and Ganesan [24] employed a first-order shear deformable semianalytical shell finite element to deal with the free vibration characteristics of isotropic and laminated orthotropic spherical caps. Kang and Leissa [25] presented a three-dimensional method of analysis for determining the free vibration frequencies and mode shapes of spherical shell segments with variable thickness. $\mathrm{Qu}$ et al. [26, 27] introduced a variational formulation for predicting the free, steady-state, and transient vibrations of composite laminated and functionally graded shells of revolution subjected to various combinations of classical and nonclassical boundary conditions. A modified variational principle in conjunction with a multisegment partitioning technique was employed to derive the formulation based on the first-order shear deformation theory. The above review of the literature reveals that problems pertaining to the free vibration analyses of closed shells subjected to classical boundaries such as free, simply-supported, and clamped and their combinations as well as elastic boundary conditions have been comprehensively studied.

In contrast to the closed shells, information available for the free vibration analysis of open shells, especially the open conical and spherical shells, is rare. Thus, the free vibration analysis of open shells based on Flügge's thin shell theory has attracted increasing research effort in the past few years. In order to properly focus on the features and emphasis of the present paper, a brief review of recent works pertaining to the vibration analyses of open shells of revolution, including the cylindrical, conical, and spherical shells, is reported below. Selmane and Lakis [28] presented a hybrid of finite element method for the dynamic and static analysis of thin, elastic, anisotropic, and nonuniform open cylindrical shells based on classical shell theories. The open shells were assumed to be freely simply supported along their curved edges and to have arbitrary straight-edge boundary conditions. Kandasamy and Singh [29] developed a formulation considering first-order shear deformation theory of shells and including rotary inertia and shear deformation to analyze the free vibration of skewed open circular cylindrical deep shells. Zhang et al. [30] presented the vibration analysis of cylindrical panels using a wave propagation method. Yu et al. [31] obtained exact solutions for open circular cylindrical shells with different combinations of primary boundary conditions using the generalized Navier method. Bardell et al. $[32,33]$ used the $h$ $p$ version of the finite element method to furnish a detailed study of the vibration characteristics of completely free, open, cylindrical, and conical isotropic shell panels. Lim and Kitipornchai [34] presented a computational investigation on the effects of subtended and vertex angles on the free vibration characteristics of open conical shell panels. Unlike the conventional approximation using a rectangular or cylindrical coordinate system, this analysis adopted a natural conical coordinate system so that any approximation in geometry was eliminated. Zhao et al. [35] analyzed the free vibration of conical panels by the mesh-free $k p$-Ritz method. Furthermore, the group in [36] extended this method to spherical panels subjected to different boundary conditions based on the three-dimensional elastic theory. Ye et al. [37] 


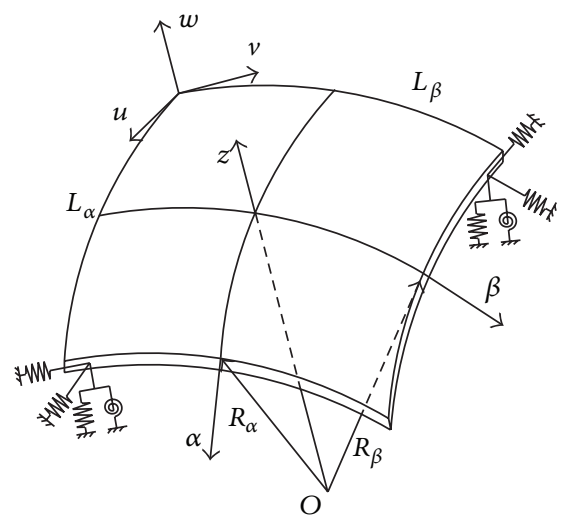

(a)

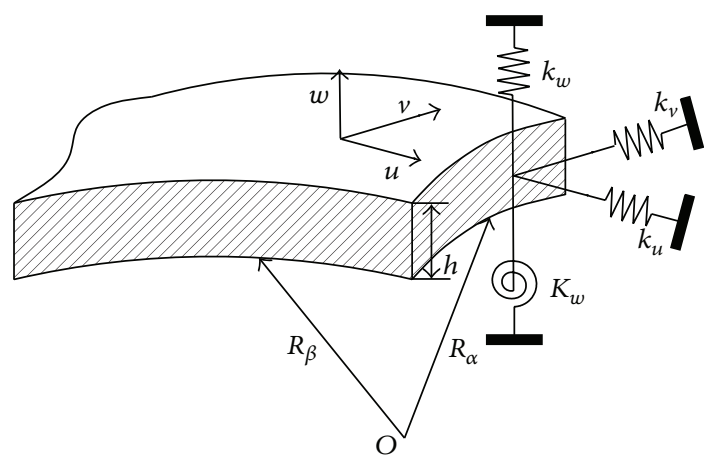

(b)

FIGURE 1: Geometry and notations of an open shell with elastically restrained edges: (a) coordinate system and (b) partial view.

studied the free vibration analysis of open shells with various boundary conditions by using the Chebyshev-Ritz method. Qatu and Asadi [38] presented the first comprehensive study of shallow shell vibrations subjected to as many as 21 possible classical boundary conditions and obtained relatively accurate results for natural frequencies of doubly curved shallow shells including spherical, cylindrical, hyperbolic, and paraboloidal shells of revolution with arbitrary boundary conditions using the Ritz method. Some other contributions are given in [39-64].

In view of the aforementioned issues and concerns, it should be emphasized that most of the researches were restricted to the single cylindrical, conical, and spherical shell or subjected to a limited sets of classical supports. The vibration behaviors of open and closed shells with varying geometrical dimensions, subjected to different combinations of classical and nonclassical boundary constraints, have until now remained unsolved. However, in practical engineering applications, the general boundary conditions are often encountered as compared to the classical boundary conditions since the support types of such structures are always complicated and variable in nature. Moreover, to the authors' best knowledge, no publications dealing with the vibration characteristics of the open and closed shells integrated with a unified, efficient, and accurate formulation have been reported.

Recently, an improved Fourier series technique proposed by $\operatorname{Li}[65,66]$ is widely used in the vibrations of beams, plates, and shells with general boundary conditions. And then Jiang et al. [67] proposed a spectrogeometric method (SGM) based on the improved Fourier series method to study different structures and their combination. In this paper, the authors present a spectro-geometric-Ritz method based on the SGM and Ritz method to solve the free vibrations of the open and closed shells with arbitrary boundary conditions. The arbitrary boundary conditions of the shells are realized by applying the artificial spring technique. The equations of motion and the related boundary equations are derived via Hamilton's principle based on Flügge's thin shell theory. Regardless of the boundary conditions, the displacement components of the thin shells are represented as the expansion series simultaneously by using the SGM, which can be described as a standard cosine series and auxiliary functions which can be expressed as a sine series. The introduction of the auxiliary functions not only removes all the potential discontinuities at the boundary, but also ensures and accelerates the convergence of the series expansions. All the expansion coefficients are considered as the generalized coordinates and determined using Rayleigh-Ritz method. By using this method, a unified vibration analysis formulation for the open and closed shells with arbitrary boundary conditions is developed without the need of changing the expression of the displacements. The reliability and accuracy of the results and the ease of implementation of the present method are validated by comparing selected cases against the FEM results and those existing in the literature. The effects of boundary conditions and the geometrical dimensions on vibration behavior of the shell are also illustrated.

\section{Theory and Formulation}

2.1. System Description. The geometry and coordinate systems for the open shell are depicted in Figure 1. The open shell under consideration is thought to be thin and of length $L_{\alpha}$, width $L_{\beta}$, and uniform thickness $h$. The middle surface of the shell where an orthogonal coordinate $(\alpha, \beta$, and $z)$ is fixed is taken as the reference surface. The radii of curvature in $\alpha$ and $\beta$ directions are represented by $R_{\alpha}$ and $R_{\beta}$, respectively. The displacement components of the shell in $\alpha, \beta$, and $z$ directions are denoted by $u, v$, and $w$, respectively. Along each end of the shell, three groups of translational springs $\left(k_{u}, k_{v}\right.$, and $\left.k_{w}\right)$ and one group of rotational springs $\left(K_{w}\right)$ which are distributed uniformly along the boundary are introduced to separately simulate the given or typical boundary conditions expressed in the form of axial force resultant, tangential force resultant, transverse force resultant, and the flexural moment resultant. Thus, the given boundary conditions can be readily achieved by assigning these springs at proper stiffness. For instance, a clamped boundary condition can be generated by simply 
setting the stiffness of all the springs equal to infinite (which is represented by a very large number). Specifically, $k_{\alpha 0}^{u}, k_{\alpha 0}^{v}$, $k_{\alpha 0}^{w}$, and $K_{\alpha 0}^{w}$ denote the set of springs distributed along the edge $\alpha=0$. Similarly, by replacing the subscript $\alpha 0$ with $\alpha L, \beta 0$, and $\beta L$, the other three sets of boundary springs at corresponding ends can be designated, respectively.

2.2. Energy Functional of the Thin Shells. According to the Kirchhoff hypothesis and Flügge's thin shell theory [4], the middle surface strains, curvature, and twist changes of the thin shell can be written in terms of displacements components as

$$
\begin{aligned}
\varepsilon_{\alpha}= & \frac{1}{A} \frac{\partial u}{\partial \alpha}+\frac{v}{A B} \frac{\partial A}{\partial \beta}+\frac{w}{R_{\alpha}}, \\
\varepsilon_{\beta}= & \frac{1}{B} \frac{\partial v}{\partial \beta}+\frac{u}{A B} \frac{\partial B}{\partial \alpha}+\frac{w}{R_{\beta}}, \\
\varepsilon_{\alpha \beta}= & \frac{A}{B} \frac{\partial}{\partial \beta}\left(\frac{u}{A}\right)+\frac{B}{A} \frac{\partial}{\partial \alpha}\left(\frac{v}{B}\right), \\
\kappa_{\alpha}= & \frac{1}{A} \frac{\partial}{\partial \alpha}\left(\frac{u}{R_{\alpha}}-\frac{1}{A} \frac{\partial w}{\partial \alpha}\right) \\
& +\frac{1}{A B}\left(\frac{v}{R_{\beta}}-\frac{1}{B} \frac{\partial w}{\partial \beta}\right) \frac{\partial A}{\partial \beta}, \\
\kappa_{\beta}= & \frac{1}{B} \frac{\partial}{\partial \beta}\left(\frac{v}{R_{\beta}}-\frac{1}{B} \frac{\partial w}{\partial \beta}\right) \\
& +\frac{1}{A B}\left(\frac{u}{R_{\alpha}}-\frac{1}{A} \frac{\partial w}{\partial \alpha}\right) \frac{\partial B}{\partial \alpha}, \\
\tau= & \frac{A}{B} \frac{\partial}{\partial \beta}\left[\frac{1}{A}\left(\frac{u}{R_{\alpha}}-\frac{1}{A} \frac{\partial w}{\partial \alpha}\right)\right] \\
& +\frac{B}{A} \frac{\partial}{\partial \alpha}\left[\frac{1}{B}\left(\frac{v}{R_{\beta}}-\frac{1}{B} \frac{\partial w}{\partial \beta}\right)\right] \\
& +\frac{1}{R_{\alpha}}\left(\frac{1}{B} \frac{\partial u}{\partial \beta}-\frac{v}{A B} \frac{\partial B}{\partial \alpha}\right) \\
& +\frac{1}{R_{\beta}}\left(\frac{1}{A} \frac{\partial v}{\partial \alpha}-\frac{u}{A B} \frac{\partial A}{\partial \beta}\right),
\end{aligned}
$$

where $\varepsilon_{\alpha}, \varepsilon_{\beta}$, and $\varepsilon_{\alpha \beta}$ indicate the strains in the middle surface; $\kappa_{\alpha}, \kappa_{\beta}$, and $\tau$ are the curvature changes; $u, v$, and $w$ denote the shell displacement components in the $\alpha, \beta$, and $z$ directions, respectively; $R_{\alpha}$ and $R_{\beta}$ represent the radii of curvature in the $\alpha, \beta$ directions as depicted in Figure 1 . The quantities $A$ and $B$ are the Lamé parameters. When the coordinate systems and geometric parameters $A, B, R_{\alpha}$, and $R_{\beta}$ are given different values, the corresponding type of shells can be obtained. In Figure 2, the specific shells constructed as the three types of curvatures, for example, cylindrical shells, conical shells, and spherical shells, are presented. According to Figure 2, the parameters $R_{\alpha}, R_{\beta}, A$, and $B$ of the above three types of shells are given as follows: (a) Cylindrical shell:

$$
\begin{aligned}
\alpha & =x ; \\
\beta & =\theta ; \\
R_{\alpha} & =1 ; \\
R_{\beta} & =R ; \\
A & =1 ; \\
B & =R .
\end{aligned}
$$

(b) Conical shell:

$$
\begin{aligned}
\alpha & =x ; \\
\beta & =\theta ; \\
R_{\alpha} & =1 ; \\
R_{\beta} & =x \tan \varphi ; \\
A & =1 ; \\
B & =x \sin \varphi .
\end{aligned}
$$

(c) Spherical shell:

$$
\begin{aligned}
\alpha & =\varphi ; \\
\beta & =\theta ; \\
R_{\alpha} & =R ; \\
R_{\beta} & =R ; \\
A & =R ; \\
B & =R \sin \varphi .
\end{aligned}
$$

According to Flügge's thin shell theory, the linear strains in the space of the thin shell are expressed as

$$
\begin{aligned}
e_{\alpha} & =\frac{1}{1+z / R_{\alpha}}\left(\varepsilon_{\alpha}+z \kappa_{\alpha}\right), \\
e_{\beta} & =\frac{1}{1+z / R_{\beta}}\left(\varepsilon_{\beta}+z \kappa_{\beta}\right), \\
\gamma_{\alpha \beta} & =\frac{1}{\left(1+z / R_{\alpha}\right)\left(1+z / R_{\beta}\right)}\left[\left(1-\frac{z^{2}}{R_{\alpha} R_{\beta}}\right) \varepsilon_{\alpha \beta}\right. \\
& \left.+z\left(1+\frac{z}{2 R_{\alpha}}+\frac{z}{2 R_{\beta}}\right) \tau\right],
\end{aligned}
$$

where $-h / 2 \leqslant z \leqslant h / 2$. According to general Hooke's law, the corresponding stresses are obtained as

$$
\left\{\begin{array}{c}
\sigma_{\alpha} \\
\sigma_{\beta} \\
\sigma_{\alpha \beta}
\end{array}\right\}=\left[\begin{array}{ccc}
Q_{11} & Q_{12} & 0 \\
Q_{21} & Q_{22} & 0 \\
0 & 0 & Q_{66}
\end{array}\right]\left\{\begin{array}{c}
e_{\alpha} \\
e_{\beta} \\
\gamma_{\alpha \beta}
\end{array}\right\},
$$




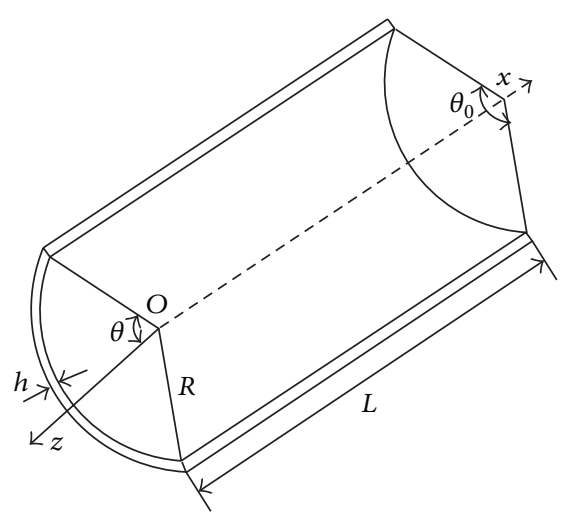

(a)

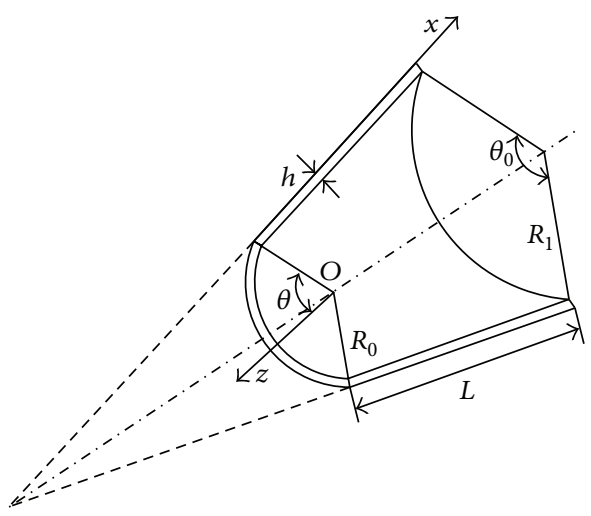

(b)

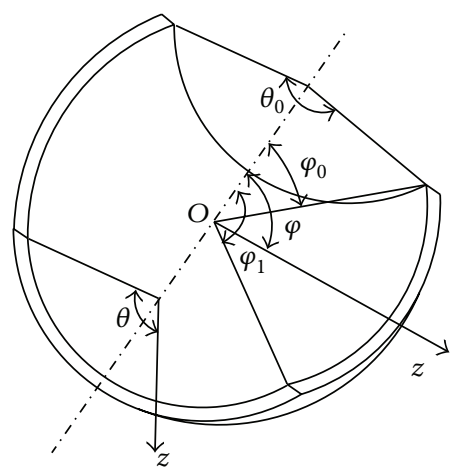

(c)

FIGURE 2: Definition of shell coordinate system: (a) cylindrical shell; (b) conical shell; and (c) spherical shell.

in which $\sigma_{\alpha}$ and $\sigma_{\beta}$ are the normal stresses; $\sigma_{\alpha \beta}$ is shear stress. $Q_{i j}(i, j=1,2,6)$ denote the constants relating stresses with strains. They are defined as

$$
\begin{aligned}
& Q_{11}=Q_{22}=\frac{E}{1-\mu^{2}}, \\
& Q_{12}=Q_{21}=\frac{\mu E}{1-\mu^{2}}, \\
& Q_{66}=\frac{E}{2(1+\mu)},
\end{aligned}
$$

where $E$ and $\mu$ are Young's modulus and Poisson's ratio, respectively.

From the theory of elasticity, the well-known expression for the strain energy stored in a shell body during elastic deformation is

$$
V_{p}=\frac{1}{2} \int_{V}\left(\sigma_{\alpha} e_{\alpha}+\sigma_{\beta} e_{\beta}+\sigma_{\alpha \beta} \gamma_{\alpha \beta}\right) d V
$$

where $\mathrm{d} V$ is the element of volume which, expressed in shell coordinates, is

$$
\mathrm{d} V=\left(1+\frac{z}{R_{\alpha}}\right)\left(1+\frac{z}{R_{\beta}}\right) A B \mathrm{~d} \alpha \mathrm{d} \beta \mathrm{d} z .
$$

Recognizing that, for a thin shell, $z / R_{i}(i=\alpha, \beta)$ is less than unity, then one can expand the quotient $1 /\left(1+z / R_{i}\right)$ into a well-known geometric series by simple division; that is,

$$
\frac{1}{1+z / R_{i}}=\sum_{n=0}^{\infty}\left(-\frac{z}{R_{i}}\right)^{n}, \quad i=\alpha, \beta
$$

Replacing $1 /\left(1+z / R_{i}\right)$ with (10), neglecting terms raised to powers of $z$ greater than two in the integrand, and substituting (1a)-(7b) and (9) into (8) result in

$$
V_{p}=\frac{E h}{2\left(1-\mu^{2}\right)} \iint\left[Q_{0}+\frac{h^{2}}{12}\left(Q_{1}+Q_{2}\right)\right] A B \mathrm{~d} \alpha \mathrm{d} \beta,
$$

where

$$
\begin{aligned}
Q_{0}= & \varepsilon_{\alpha}^{2}+\varepsilon_{\beta}^{2}+2 \mu \varepsilon_{\alpha} \varepsilon_{\beta}+\frac{1-\mu}{2} \varepsilon_{\alpha \beta}^{2}, \\
Q_{1}= & \kappa_{\alpha}^{2}+\kappa_{\beta}^{2}+2 \mu \kappa_{\alpha} \kappa_{\beta}+\frac{1-\mu}{2} \tau^{2}, \\
Q_{2}= & -2\left(\frac{1}{R_{\alpha}}-\frac{1}{R_{\beta}}\right)\left(\varepsilon_{\alpha} \kappa_{\alpha}-\varepsilon_{\beta} \kappa_{\beta}\right) \\
& -\frac{1-\mu}{2}\left(\frac{1}{R_{\alpha}}+\frac{1}{R_{\beta}}\right) \varepsilon_{\alpha \beta} \tau \\
& +\left(\frac{1}{R_{\alpha}}-\frac{1}{R_{\beta}}\right)\left(\frac{\varepsilon_{\alpha}^{2}}{R_{\alpha}}-\frac{\varepsilon_{\beta}^{2}}{R_{\beta}}\right) \\
& +\frac{1-\mu}{2}\left(\frac{1}{R_{\alpha}^{2}}+\frac{1}{R_{\beta}^{2}}-\frac{1}{R_{\alpha} R_{\beta}}\right) \varepsilon_{\alpha \beta}^{2},
\end{aligned}
$$

and the maximum kinetic energy of the thin shell can be defined as

$$
T=\frac{\rho h}{2} \iint\left(\dot{w}^{2}+\dot{u}^{2}+\dot{v}^{2}\right) A B \mathrm{~d} \alpha \mathrm{d} \beta,
$$

where the dot above a variable represents differentiation with respect to time. Since the value of the kinetic energy is maximum, the kinetic energy expression in (13) can be rewritten as

$$
T=\frac{\rho h \omega^{2}}{2} \iint\left(w^{2}+u^{2}+v^{2}\right) A B \mathrm{~d} \alpha \mathrm{d} \beta .
$$

In (11)-(14), $E, \mu$, and $\rho$, respectively, are Young's modulus, Poisson ratio, and density of the shell. $\omega$ indicates the angular frequency of the shell.

2.3. Arbitrary Boundary Conditions and Continuity Conditions. In order to simulate the arbitrary boundary conditions, three groups of translational restraining springs $\left(k^{w}, k^{u}\right.$, and $k^{v}$ ) and one group of rotational restraining springs $\left(K^{w}\right)$ 
which are arranged uniformly along each boundary with independent stiffness are introduced to separately simulate the given or typical boundary conditions expressed in the form of axial force resultant, tangential force resultant, transverse force resultant, and the flexural moment resultant, which is shown in Figure 1. Thus, the given boundary conditions can be readily achieved by assigning these springs at proper stiffness. For instance, a clamped boundary condition can be generated by simply setting the stiffness of all the springs equal to infinite (which is represented by a very large number). Therefore, the general boundary conditions for an elastically restrained shell can be described as follows:

At edge $\alpha=0$,

$$
\begin{aligned}
k_{\alpha 0}^{u} u & =N_{\alpha}, \\
k_{\alpha 0}^{v} v & =N_{\alpha \beta}+\frac{M_{\alpha \beta}}{R_{\beta}}, \\
k_{\alpha 0}^{w} w & =Q_{\alpha}+\frac{\partial M_{\alpha \beta}}{\partial \beta}, \\
K_{\alpha 0}^{w} \frac{\partial w}{\partial \alpha} & =-M_{\alpha} .
\end{aligned}
$$

At edge $\alpha=L_{\alpha}$,

$$
\begin{aligned}
k_{\alpha L}^{u} u & =-N_{\alpha}, \\
k_{\alpha L}^{v} v & =-N_{\alpha \beta}-\frac{M_{\alpha \beta}}{R_{\beta}}, \\
k_{\alpha L}^{w} w_{c} & =-Q_{\alpha}-\frac{\partial M_{\alpha \beta}}{\partial \beta}, \\
K_{\alpha L}^{w} \frac{\partial w}{\partial \alpha} & =M_{\alpha} .
\end{aligned}
$$

At edge $\beta=0$,

$$
\begin{aligned}
k_{\beta 0}^{u} u & =N_{\alpha \beta}+\frac{\partial M_{\alpha \beta}}{\partial R_{\alpha}}, \\
k_{\beta 0}^{v} v & =N_{\beta}, \\
k_{\beta 0}^{w} w & =Q_{\beta}+\frac{\partial M_{\alpha \beta}}{\partial \alpha}, \\
K_{\beta 0}^{w} \frac{\partial w}{\partial \beta} & =-M_{\beta} .
\end{aligned}
$$

At edge $\beta=L_{\beta}$,

$$
\begin{aligned}
& k_{\beta L}^{u} u=-N_{\alpha \beta}-\frac{M_{\alpha \beta}}{R_{\alpha}}, \\
& k_{\beta L}^{v} v=-N_{\beta},
\end{aligned}
$$

$$
\begin{aligned}
k_{\beta L}^{w} w & =-Q_{\beta}-\frac{\partial M_{\alpha \beta}}{\partial \alpha}, \\
K_{\beta L}^{w} \frac{\partial w}{\partial \beta} & =M_{\beta},
\end{aligned}
$$

where $N_{\alpha}, N_{\alpha \beta}$, and $Q_{\alpha}$ denote the force resultants acting on the face perpendicular to the $\alpha$-axis. $N_{\beta}$, $N_{\beta \alpha}\left(N_{\beta \alpha}=N_{\alpha \beta}\right)$, and $Q_{\alpha}$ are the force resultants on the face perpendicular to the $\beta$-axis. Similarly, $M_{\alpha}, M_{\beta}$, and $M_{\alpha \beta}$ are the moment resultants on the corresponding face. $N_{\alpha}, N_{\beta}$, and $N_{\alpha \beta}$ are the normal and in-plane shear force resultants; $Q_{\alpha}$ and $Q_{\beta}$ denote the transverse shear force; $M_{\alpha}, M_{\beta}$, and $M_{\alpha \beta}$ represent the bending and twisting moment resultants.

The force and moment resultants are obtained by integrating the stresses over the shell thickness:

$$
\begin{aligned}
& {\left[\begin{array}{c}
N_{\alpha} \\
N_{\beta} \\
N_{\alpha \beta}
\end{array}\right]=\int_{-h / 2}^{h / 2}\left[\begin{array}{c}
\sigma_{\alpha} \\
\sigma_{\beta} \\
\sigma_{\alpha \beta}
\end{array}\right] \mathrm{d} z,} \\
& {\left[\begin{array}{c}
M_{\alpha} \\
M_{\beta} \\
M_{\alpha \beta}
\end{array}\right]=\int_{-h / 2}^{h / 2}\left[\begin{array}{c}
\sigma_{\alpha} \\
\sigma_{\beta} \\
\sigma_{\alpha \beta}
\end{array}\right] z \mathrm{~d} z,} \\
& {\left[\begin{array}{c}
Q_{\alpha} \\
Q_{\beta}
\end{array}\right]=\int_{-h / 2}^{h / 2}\left[\begin{array}{c}
\sigma_{\alpha z} \\
\sigma_{\beta z}
\end{array}\right] \mathrm{d} z .}
\end{aligned}
$$

By carrying the integration of stresses over the cross section and integrating the moments of the in-plane stresses over the thickness, the force and moment resultants relations to the strains in the middle surface and curvature changes are defined as

$$
\begin{aligned}
N_{\alpha} & =\frac{E h}{1-\mu^{2}}\left(\varepsilon_{\alpha}+\mu \varepsilon_{\beta}\right), \\
N_{\beta} & =\frac{E h}{1-\mu^{2}}\left(\varepsilon_{\beta}+\mu \varepsilon_{\alpha}\right), \\
N_{\alpha \beta} & =\frac{E h}{2(1+\mu)} \varepsilon_{\alpha \beta}, \\
M_{\alpha} & =\frac{E h^{3}}{12\left(1-\mu^{2}\right)}\left(\kappa_{\alpha}+\mu \kappa_{\beta}\right), \\
M_{\beta} & =\frac{E h^{3}}{12\left(1-\mu^{2}\right)}\left(\kappa_{\beta}+\mu \kappa_{\alpha}\right), \\
M_{\alpha \beta} & =\frac{E h^{3}}{24(1+\mu)} \tau .
\end{aligned}
$$




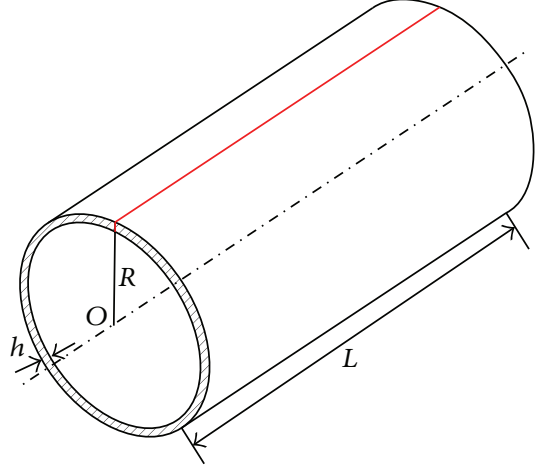

_ Coupling circumferential edge

(a)

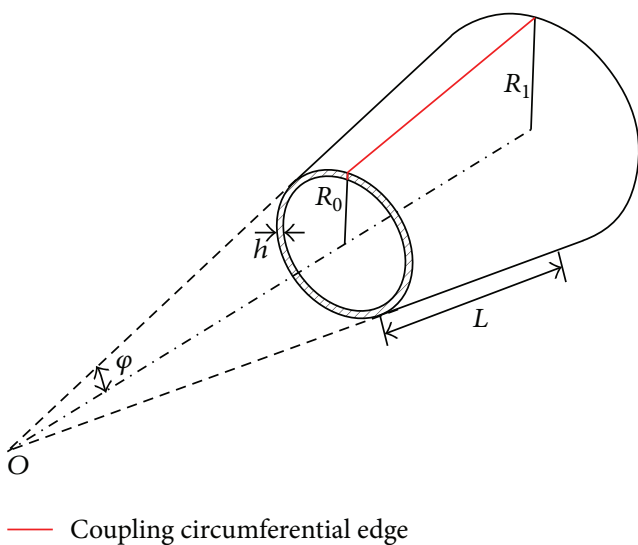

(b)

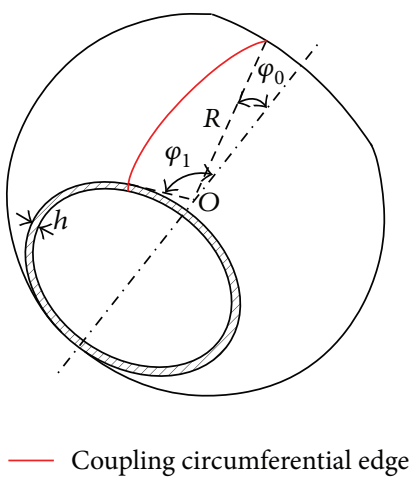

(c)

FIGURE 3: The sketches of the closed shells with coupling circumferential edge: (a) cylindrical shell; (b) conical shell; (c) spherical shell.

Thus, the total potential energy of the spring restrained shell stored in the boundary springs can be expressed as

$$
\begin{aligned}
V_{b} & =\frac{1}{2} \int\left\{\left[k_{\beta 0}^{w} w^{2}+k_{\beta 0}^{u} u^{2}+k_{\beta 0}^{v} v^{2}\right.\right. \\
& \left.+K_{\beta 0}^{w}\left(\frac{v}{R_{\beta}}-\frac{\partial w}{\partial \beta}\right)^{2}\right]_{\beta=0}+\left[k_{\beta L}^{w} w^{2}+k_{\beta L}^{u} u^{2}\right. \\
& \left.\left.+k_{\beta L}^{v} v^{2}+K_{\beta L}^{w}\left(\frac{v}{R_{\beta}}-\frac{\partial w}{\partial \beta}\right)^{2}\right]_{\beta=L_{\beta}}\right\} A \mathrm{~d} \alpha+\frac{1}{2} \\
& \cdot \int\left\{\left[k_{\alpha 0}^{w} w^{2}+k_{\alpha 0}^{u} u^{2}+k_{\alpha 0}^{v} v^{2}\right.\right. \\
& \left.+K_{\alpha 0}^{w}\left(\frac{u}{R_{\alpha}}-\frac{\partial w}{\partial \alpha}\right)^{2}\right]_{\alpha=0}+\left[k_{\alpha L}^{w} w^{2}+k_{\alpha L}^{u} u^{2}\right. \\
& \left.\left.+k_{\alpha L}^{v} v^{2}+K_{\alpha L}^{w}\left(\frac{u}{R_{\alpha}}-\frac{\partial w}{\partial \alpha}\right)^{2}\right]_{\alpha=L_{\alpha}}\right\} B \mathrm{~d} \beta .
\end{aligned}
$$

As indicated in the mathematical expressions of boundary conditions, all the force and moment resultants of the open shells are restrained by utilized springs. Therefore, arbitrary boundary constraints can be achieved by varying the values of spring's stiffness. The classical boundary conditions specially can be simulated by varying the stiffness of the boundary springs to be extremely large or extremely small.

When all of the energy expressions are prepared, the complete solution can be obtained by using the RayleighRitz method. The total energy functional of the open shell is taken as the sum of the energy contributions from the shell, which is composed of the strain energy and the kinetic energy, and boundary constraints. Thus, the Lagrangian energy functional $(L)$ of the open shell can be expressed as

$$
L=V_{p}+V_{b}-T \text {. }
$$

As mentioned earlier, a closed shell can be mathematically viewed as a special case when the inclusion or circumferential angle is set equal to $2 \pi$, as shown in Figure 3. However, this simple mathematical operation alone cannot automatically ensure a complete transition of the open shell into a closed shell; that is, the continuities of the displacements and their derivatives at $\theta=0$ and $\theta=2 \pi$ are not necessarily the same. To overcome this problem, a set of coupling springs $\left(k_{c}^{u}, k_{c}^{v}\right.$, $k_{c}^{w}$, and $K_{c}^{w}$ ), which are uniformly distributed at the common edge, will be adopted to enforce the continuity conditions of displacements, slope, forces, and bending moment at the junction in circumference. The presence of these coupling springs can be accounted for by the potential energies:

$$
\begin{aligned}
V_{c} & =\frac{1}{2} \int\left[k_{c}^{w}\left(\left.w\right|_{\theta=0}-\left.w\right|_{\theta=2 \pi}\right)^{2}+k_{c}^{u}\left(\left.u\right|_{\theta=0}\right.\right. \\
& \left.-\left.u\right|_{\theta=2 \pi}\right)^{2}+k_{c}^{v}\left(\left.v\right|_{\theta=0}-\left.v\right|_{\theta=2 \pi}\right)^{2}+K_{c}^{w}\left(\left.\frac{v}{R_{\beta}}\right|_{\theta=0}\right. \\
& \left.\left.-\left.\frac{\partial w}{\partial \beta}\right|_{\theta=0}-\left.\frac{v}{R_{\beta}}\right|_{\theta=2 \pi}+\left.\frac{\partial w}{\partial \beta}\right|_{\theta=2 \pi}\right)^{2}\right] A \mathrm{~d} \alpha
\end{aligned}
$$

where $k_{c}^{w}, k_{c}^{u}$, and $k_{c}^{v}$ represent the stiffness for translational coupling springs; $K_{c}^{w}$ is the stiffness for rotational coupling springs.

Therefore, arbitrary coupling conditions can be obtained by varying the values of coupling spring's stiffness. The discontinuity and continuity of the displacement and slope specially at the common edge can be achieved by varying the stiffness of the boundary springs to be extremely small and extremely large, which can simulate the open shell with the circumferential angle $\theta=2 \pi$ and the closed shell, respectively. In fact, these two cases are different and the two cases will get different vibration behavior. The function of connective springs at the interface for the continuity of 
the displacement and slope is embodied in the Lagrangian energy functional $(L)$ of the closed shell:

$$
L=V_{p}+V_{b}+V_{c}-T
$$

In fact, as the open shell is expanded to the closed one, the boundary restraint spring's stiffness at the edges $\theta=0$ and $\theta=2 \pi$ should be set to zero and the coupling spring's stiffness should be set infinitely large.

\subsection{Admissible Displacement Functions and Solution Proce-} dure. An admissible function for displacement is essential to achieve an accurate and convergent solution in the RayleighRitz procedure. However, the original Fourier series expression only applies to a few simple boundary conditions and can lead to unavoidable convergence problem for other boundary conditions, thus limiting the applications of the Fourier series to only a few ideal boundary conditions [65]. Take a beam problem as an example. The governing equations for free vibration of a general supported Euler beam are obtained as

$$
D \frac{\partial^{4} w(x)}{\partial x^{4}}-\rho A \omega^{2} w(x)=0
$$

where $D, \rho$, and $A$ are, respectively, the flexural rigidity, the mass density, and the cross-sectional area of the beam; $\omega$ is frequency in radian. From (25), we can know that the displacement solution $w(x)$ on a beam of length $L$ is required to have up to the fourth derivatives; that is, $w(x) \in C^{3}$. Generally, the displacement function $w(x)$ defined on an interval $[0, L]$ can be expanded into a Fourier series inside the interval excluding boundary points:

$$
w(x)=\sum_{m=0}^{\infty} A_{m} \cos \left(\frac{m \pi x}{L}\right), \quad 0<x<L
$$

where $A_{m}$ are the expansion coefficients. From (26), it can be seen that the transverse displacement function $w(x)$ can be viewed as a part of an even function defined on the interval $[-L, L]$, as shown in Figure 4. Thus, the Fourier cosine series is able to correctly converge to $w(x)$ at any point over $[0, L]$. However, its first derivative $w^{\prime}(x)$ is an odd function on $[-L, L]$ leading to a jump at end locations. The corresponding Fourier expansion of $w^{\prime}(x)$ continues on $[0, L]$ and can be differentiated term-by-term only if $w^{\prime}(0)=w^{\prime}(L)=0$. Thus, its Fourier series expansion (sine series) will accordingly have a convergence problem due to the discontinuity at end points when $w^{i}(x)$ is required to have up to the first-derivative continuity.

Recently, Li $[65,66]$ proposed an improved Fourier series technique to overcome this problem; in this technique, the displacement function $w(x)$ considers a new function:

$$
w(x)=\bar{w}(x)+P(x)=\sum_{m=0}^{\infty} A_{m} \cos \left(\frac{m \pi x}{L}\right)+P(x)
$$

$$
0 \leq x \leq L
$$

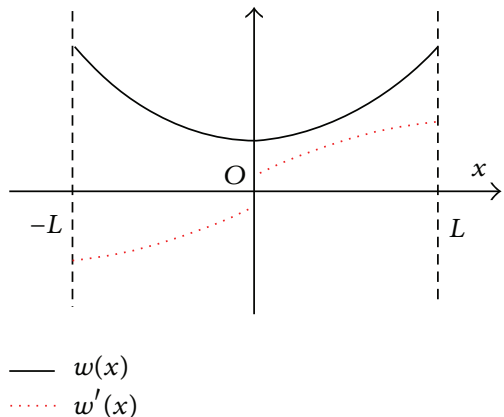

Figure 4: An illustration of the possible discontinuities of the displacement and its derivative at the end points.

where auxiliary function $P(x)$ in (27) represents an arbitrary continuous function that, regardless of boundary conditions equations (15)-(20b), is always chosen to satisfy the following equations:

$$
\begin{gathered}
P^{\prime}(0)=w^{\prime}(0)=\alpha_{0}, \\
P^{\prime}(a)=w^{\prime}(a)=\alpha_{1}, \\
P^{\prime \prime \prime}(0)=w^{\prime \prime \prime}(0)=\beta_{0}, \\
P^{\prime \prime \prime}(a)=w^{\prime \prime \prime}(a)=\beta_{1} .
\end{gathered}
$$

In (28a), (28b), (28c), and (28d), the actual values $\alpha_{0}, \alpha_{1}$, $\beta_{0}$, and $\beta_{1}$ of the first and third derivatives at the boundaries need to be determined from the given boundary conditions equations (15)-(20b). The function $P(x)$ is here introduced to take care of the potential discontinuities of the (original) displacement function and its derivatives at the end points. Essentially, $\bar{w}(x)$ represents a residual beam function which is continuous over $[0, L]$ and has zero slopes at both ends. Apparently, the cosine series with the auxiliary function $P(x)$ employed representation of $w(x)$ is able to converge correctly to the function itself and its first derivative at every point on the beam. Thus, based on the above analysis, $P(x)$ can be understood as a continuous function that satisfies (28a), (28b), (28c), and (28d); its form is not a concern but must be a closed form and sufficiently smooth on the interval $[0, L]$ of the beam in order to meet the requirements provided by the continuity conditions and boundary constraints. Therefore, the addition of the auxiliary function $P(x)$ will have two immediate benefits: (1) the Fourier series expansion will be applicable to any boundary condition; (2) the Fourier series solution can be drastically improved regarding its accuracy convergence.

According to the above analysis, it is simple and convenient to expand the displacement field of beams to one of the shells. In this paper, the function $P(x)$ is chosen in a trigonometric series form by using SGM. Thus, the displacement field of the thin shells can be described by 
the SGM and then the detailed expressions of the displacements in $z, \alpha$, and $\beta$ directions are given as

$$
\begin{aligned}
& w(\alpha, \beta)=\sum_{m=0}^{\infty} \sum_{n=0}^{\infty} W_{m n}^{1} \cos \lambda_{m} \alpha \cos \lambda_{n} \beta \\
& +\sum_{m=0}^{\infty} \sum_{n=1}^{4} W_{m n}^{2} \cos \lambda_{m} \alpha \sin \lambda_{n} \beta \\
& +\sum_{m=1}^{4} \sum_{n=0}^{\infty} W_{m n}^{3} \cos \lambda_{n} \beta \sin \lambda_{n} \alpha \\
& +\sum_{m=1}^{4} \sum_{n=1}^{4} W_{m n}^{4} \sin \lambda_{m} \alpha \sin \lambda_{n} \beta, \\
& u(\alpha, \beta)=\sum_{m=0}^{\infty} \sum_{n=0}^{\infty} U_{m n}^{1} \cos \lambda_{m} \alpha \cos \lambda_{n} \beta \\
& +\sum_{m=0}^{\infty} \sum_{n=1}^{2} U_{m n}^{2} \cos \lambda_{m} \alpha \sin \lambda_{n} \beta \\
& +\sum_{m=1}^{2} \sum_{n=0}^{\infty} U_{m n}^{3} \cos \lambda_{n} \beta \sin \lambda_{m} \alpha \\
& +\sum_{m=1}^{2} \sum_{n=1}^{2} U_{m n}^{4} \sin \lambda_{m} \alpha \sin \lambda_{n} \beta, \\
& v(\alpha, \beta)=\sum_{m=0}^{\infty} \sum_{n=0}^{\infty} V_{m n}^{1} \cos \lambda_{m} \alpha \cos \lambda_{n} \beta \\
& +\sum_{m=0}^{\infty} \sum_{n=1}^{2} V_{m n}^{1} \cos \lambda_{m} \alpha \sin \lambda_{n} \beta \\
& +\sum_{m=1}^{2} \sum_{n=0}^{\infty} V_{m n}^{3} \cos \lambda_{n} \beta \sin \lambda_{m} \alpha \\
& +\sum_{m=1}^{2} \sum_{n=1}^{2} V_{m n}^{4} \sin \lambda_{m} \alpha \sin \lambda_{n} \beta
\end{aligned}
$$

where $\lambda_{m}=m \pi / L_{\alpha}, \lambda_{n}=n \pi / L_{\beta}, W_{m n}^{1}, W_{m n}^{2}, W_{m n}^{3}, W_{m n}^{4}$, $U_{m n}^{1}, U_{m n}^{2}, U_{m n}^{3}, U_{m n}^{4}, V_{m n}^{1}, V_{m n}^{2}, V_{m n}^{3}$, and $V_{m n}^{4}$ are the expansion coefficients for the shells and they can be solved by the Rayleigh-Ritz procedure.

For thin shells, the thickness is assumed to be negligible compared to length or radii of curvature of the shell and the normal to the middle surfaces are considered always to be straight and normal to the middle surface. Under these assumptions, Rayleigh-Ritz energy method will be used to establish the theoretical formulation of the thin shells based on Flügge's thin shell theory.
Since the energy expressions and admissible function of the shell have been established, the remaining task is to determine the unknown coefficients and supplemented coefficients of displacement functions. The Lagrangian energy functions $(L)$ of the open and closed shell have been expressed in (22) and (24). Thus, the Lagrangian energy function can be expressed with displacement functions by substituting (11), (14), (21), and (23) together with the admissible functions defined in (29a), (29b), and (29c) into (22) and (24). The undetermined coefficients of the expansion series are considered the generalized coordinates and then, using the Rayleigh-Ritz method, the Lagrangian expression is minimized by taking its derivatives with respect to these coefficients:

$$
\frac{\partial L}{\partial \vartheta}=0, \quad \vartheta=W_{m n}, U_{m n}, V_{m n}
$$

where $\vartheta$ denotes the undetermined coefficients including $W_{m n}, U_{m n}$, and $V_{m n}$. Since the displacement functions of the shell structure are truncated at $m=M$ and $n=N$ to obtain the results with acceptable accuracy, a total of $(M+5) *(N+$ $5)+2 *(M+3) *(N+3)$ equations are obtained.

By substituting (22) and (24) into (30), it can be summed up in a matrix form as follows:

$$
\left(\mathbf{K}-\omega^{2} \mathbf{M}\right) \mathbf{E}=\mathbf{0},
$$

where $\mathbf{K}$ denotes the stiffness matrix for the structure and $\mathbf{M}$ is the mass matrix. The unknown coefficients in the displacement expressions can be described in the vector form as E. They can be expressed separately as

$$
\begin{aligned}
& \mathbf{K}=\left[\begin{array}{ccc}
\mathbf{K}_{w w} & \mathbf{K}_{w u} & \mathbf{K}_{w v} \\
& \mathbf{K}_{u u} & \mathbf{K}_{u v} \\
\text { symm } & & \mathbf{K}_{v v}
\end{array}\right] \text {, } \\
& \mathbf{M}=\operatorname{diag}\left[\begin{array}{lll}
\mathbf{M}_{w w} & \mathbf{M}_{u u} & \mathbf{M}_{v v}
\end{array}\right] \\
& \mathbf{E}=\left[\mathbf{W}_{m n}, \mathbf{U}_{m n}, \mathbf{V}_{m n}\right]^{T}, \\
& \mathbf{W}_{m n}=\left[W_{0,0}^{1}, W_{0,1}^{1}, \ldots, W_{m, n}^{1}, \ldots, W_{M, N}^{1}, W_{0,1}^{2}, \ldots, W_{0,4}^{2},\right. \\
& W_{1,1}^{2}, \ldots, W_{m, 4}^{2}, \ldots, W_{M, 4}^{2}, W_{1,0}^{3}, W_{1,1}^{3}, \ldots, W_{1, N}^{3}, W_{2,0}^{3} \text {, } \\
& \left.\ldots, W_{4, N}^{3}, W_{1,1}^{4}, \ldots W_{1,4}^{4}, W_{2,1}^{4}, \ldots, W_{2,4}^{4}, \ldots, W_{4,4}^{4}\right]^{T} \text {, } \\
& \mathbf{U}_{m n}=\left[U_{0,0}^{1}, U_{0,1}^{1}, \ldots, U_{m, n}^{1}, \ldots, U_{M, N}^{1}, U_{0,1}^{2}, U_{0,2}^{2}, U_{1,1}^{2}, \ldots,\right. \\
& U_{m, 2}^{2}, \ldots, U_{M, 2}^{2}, U_{1,0}^{3}, U_{1,1}^{3}, \ldots, U_{1, N}^{3}, U_{2,0}^{3}, \ldots, U_{2, N}^{3}, U_{1,1}^{4} \text {, } \\
& \left.U_{1,2}^{4}, U_{2,1}^{4}, U_{2,2}^{4}\right]^{T} \text {, } \\
& \mathbf{V}_{m n}=\left[V_{0,0}^{1}, V_{0,1}^{1}, \ldots, V_{m, n}^{1}, \ldots, V_{M, N}^{1}, V_{0,1}^{2}, V_{0,2}^{2}, V_{1,1}^{2}, \ldots,\right. \\
& V_{m, 2}^{2}, \ldots, V_{M, 2}^{2}, V_{1,0}^{3}, V_{1,1}^{3}, \ldots, V_{1, N}^{3}, V_{2,0}^{3}, \ldots, V_{2, N}^{3}, V_{1,1}^{4} \text {, } \\
& \left.V_{1,2}^{4}, V_{2,1}^{4}, V_{2,2}^{4}\right]^{T} \text {. }
\end{aligned}
$$


Obviously, the natural frequencies and eigenvectors can now be readily obtained by solving a standard matrix eigenvalue problem. Since the components of each eigenvector are actually the coefficients of the expansion series, the corresponding mode shapes can be directly determined from (35a), (35b), and (35c). In other words, once the coefficient eigenvector $\mathbf{E}$ is determined for a given frequency, the displacement functions of the shell structure can be determined by substituting the coefficients into (29a), (29b), and (29c). When the forced vibration is involved, by adding the work done by external force in the Lagrangian energy function and summing the loading vector $\mathbf{F}$ on the right side of (31), the characteristic equation for the forced vibration of the shell can readily be obtained.

As mentioned previously, the present method can obtain vibration characteristics for the shells with arbitrary boundary conditions including uniform and nonuniform restraints. Thus, another interest of this paper is focused on the nonuniform boundary restraints, and therefore, as the considerably prevalent cases, the point supported case will be considered here. For the point supported case, instead of being uniformly distributed around the shell ends, the boundary springs are located at discrete points. In this way, the total energy stored by boundary springs is given by

$$
\begin{aligned}
V_{b}^{\text {points }}=\frac{1}{2} & \sum_{s=0}^{N S}\left\{k_{\beta 0, s}^{w} w\left(\alpha_{s}, \beta_{s}\right)^{2}+k_{\beta 0, s}^{u} u\left(\alpha_{s}, \beta_{s}\right)^{2}+k_{\beta 0, s}^{v} v\left(\alpha_{s}, \beta_{s}\right)^{2}+K_{\beta 0, s}^{w}\left[\frac{v\left(\alpha_{s}, \beta_{s}\right)}{R_{\beta}}-\frac{\partial w\left(\alpha_{s}, \beta_{s}\right)}{\partial \beta}\right]^{2}\right\}_{\beta_{s}=0}+\frac{1}{2} \\
& \cdot \sum_{s=0}^{N S}\left\{k_{\beta L, s}^{w} w\left(\alpha_{s}, \beta_{s}\right)^{2}+k_{\beta L, s}^{u} u\left(\alpha_{s}, \beta_{s}\right)^{2}+k_{\beta L, s}^{v} v\left(\alpha_{s}, \beta_{s}\right)^{2}+K_{\beta L, s}^{w}\left[\frac{v\left(\alpha_{s}, \beta_{s}\right)}{\left.\left.R_{\beta}-\frac{\partial w\left(\alpha_{s}, \beta_{s}\right)}{\partial \beta}\right]^{2}\right\}+\frac{1}{2}}\right.\right. \\
& \cdot \sum_{s=0}^{N S}\left\{k_{\alpha 0, s}^{w} w\left(\alpha_{s}, \beta_{s}\right)^{2}+k_{\alpha 0, s}^{u} u\left(\alpha_{s}, \beta_{s}\right)^{2}+k_{\alpha 0, s}^{v} v\left(\alpha_{s}, \beta_{s}\right)^{2}+K_{\alpha 0, s}^{w}\left[\frac{u\left(\alpha_{s}, \beta_{s}\right)}{\left.R_{\alpha}-\frac{\partial w\left(\alpha_{s}, \beta_{s}\right)}{\partial \alpha}\right\}_{\alpha_{s}=0}^{2}+\frac{1}{2}}\right.\right. \\
& \cdot \sum_{s=0}^{N S}\left\{k_{\alpha L, s}^{w} w\left(\alpha_{s}, \beta_{s}\right)^{2}+k_{\alpha L, s}^{u} u\left(\alpha_{s}, \beta_{s}\right)^{2}+k_{\alpha L, s}^{v} v\left(\alpha_{s}, \beta_{s}\right)^{2}+K_{\alpha L, s}^{w}\left[\frac{u\left(\alpha_{s}, \beta_{s}\right)}{\left.\left.R_{\alpha}-\frac{\partial w\left(\alpha_{s}, \beta_{s}\right)}{\partial \alpha}\right]^{2}\right\}_{\alpha_{s}=L_{\alpha}},}\right.\right.
\end{aligned}
$$

where NS means the total number of restrained points along each boundary end. $k_{\alpha 0, s}^{w}, k_{\alpha 0, s}^{u}, k_{\alpha 0, s}^{v}$, and $K_{\alpha 0, s}^{w}$ are the stiffness of the four groups of boundary springs at the $s$ th point at $\alpha_{s}=0$. Similarly, $k_{\alpha L, s}^{w}, k_{\alpha L, s}^{u}, k_{\alpha L, s}^{v}$, and $K_{\alpha L, s}^{w}$ denote the stiffness of corresponding springs at $\alpha_{s}=L_{\alpha} ; k_{\beta 0, s}^{w}$, $k_{\beta 0, s}^{u}, k_{\beta 0, s}^{v}$, and $K_{\beta 0, s}^{w}$ express the stiffness of corresponding springs at $\beta_{s}=0$; and $k_{\beta L, s}^{w}, k_{\beta L, s}^{u}, k_{\beta L, s}^{v}$, and $K_{\beta L, s}^{w}$ represent the stiffness of corresponding springs at $\beta_{s}=L_{\beta}$. The coordinates of the $s$ th point are represented by $\left(\alpha_{s}, \beta_{s}\right)$. It can be seen from (36) that the numbers and locations of the boundary point supports are little limited, making it available for both equally spaced or asymmetrically located cases. Similar to the uniform boundary restraint case, by solving characteristic equation (31) for the nonuniform boundary conditions, the eigenvalues can be obtained by replacing the energy expressions of uniform boundary restraints, the integrals in (21), with those of the nonuniform boundary restraints, in (36).

\section{Results and Discussion}

In this section, a systematic comparison between the current solutions and theoretical results published by other researchers or finite element method (FEM) results is carried out to verify the excellent accuracy, reliability, and feasibility of the spectro-geometric-Ritz method. A comprehensive study about the effects on the vibration behavior of various boundary conditions and geometric parameters is also presented. The discussion is arranged as follows. Firstly, the convergence of the current solution is checked. In addition, the influence of the stiffness of boundary spring components is studied. Secondly, the accuracy and reliability of the unified formulation of the open and closed shells with various arbitrary boundary conditions and structural parameters are examined. Finally, the effects on the vibration behavior of the various boundary restraint parameters and structural geometric parameters are investigated.

For conveniently referring to the classical boundary conditions, F, SD, S, and C denote, respectively, free, Sheardiaphragm, simply supported, and clamped restraints. In addition to that, a four-letter (two-letter) string is applied for the open (closed) shells to represent the boundary condition. For examples, F-SD-S-C identifies the open shell with edges $\alpha=0, \beta=0, \alpha=L_{\alpha}$, and $\beta=L_{\beta}$ having free, Shear-diaphragm, simply supported, and clamped boundary conditions, respectively. Similarly, C-F represents the closed shell with edges $\alpha=0$ and $\alpha=L_{\alpha}$ having clamped and free boundary restraints. In the following discussion, vibration frequencies of the shell structure with classical boundary conditions, general elastic boundary conditions, and their combinations will be presented. Taking edge $\alpha=0$ as a case, the corresponding spring stiffness parameters for three types of classical boundary conditions and three types of elastic 
boundary conditions, which are commonly encountered in engineering practices, are given as follows:

$$
\text { B.C. }\left\{\begin{array}{l}
\text { F: } k_{\alpha 0}^{u}=0, k_{\alpha 0}^{v}=0, k_{\alpha 0}^{w}=0, K_{\alpha 0}^{w}=0 \\
\text { SD: } k_{\alpha 0}^{u}=0, k_{\alpha 0}^{v}=10^{10} D, k_{\alpha 0}^{w}=10^{10} D, K_{\alpha 0}^{w}=0 \\
\text { C: } k_{\alpha 0}^{u}=10^{10} D, k_{\alpha 0}^{v}=10^{10} D, k_{\alpha 0}^{v}=10^{10} D, k_{\alpha 0}^{w}=10^{10} D, K_{\alpha 0}^{w}=0 \\
\mathrm{E}^{1}: k_{\alpha 0}^{u}=10^{4} D, k_{\alpha 0}^{v}=10^{10} D \\
\mathrm{E}^{2}: k_{\alpha 0}^{u}=10^{10} D, k_{\alpha 0}^{v}=10^{10} D, K_{\alpha 0}^{w}=10^{10} D \\
\mathrm{E}^{3}: k_{\alpha 0}^{u}=10^{10} D, k_{\alpha 0}^{w}=10^{10} D, K_{\alpha 0}^{w}=10^{10} D \\
{ }^{10} D, k_{\alpha 0}^{w}=10^{4} D, K_{\alpha 0}^{w}=10^{10} D,
\end{array}\right.
$$

where $D=E h^{3} / 12\left(1-\mu^{2}\right)$ is the transverse flexural stiffness of the shell. The unit of the translational restraining spring is $\mathrm{N} / \mathrm{m}$, the unit of the rotational spring is $\mathrm{Nm} / \mathrm{rad}$, and the spring's stiffness values are expressed in a unit length along the edge. The appropriateness of defining the classical boundary conditions of shell structures in terms of boundary spring's stiffness will be verified by several examples given in following discussion.

3.1. Convergence and Stiffness Value Study. Since the expansion series is numerically truncated and only finite terms are counted in actual calculations, the proposed method should be understood as a solution with arbitrary precision. For the sake of validating the convergence of present method firstly, natural frequencies of the open shell, that is, cylindrical shell, conical shell, and spherical shell, subject to different truncated configurations, are carried out in this subsection. In order to compare with those in other literatures, the dimensions of the shell structures used for the analysis are the following: for cylindrical shell, $E=210 \mathrm{GPa}, \mu=0.3$, $\rho=7800 \mathrm{~kg} / \mathrm{m}^{3}, R=2 \mathrm{~m}, L=3 \mathrm{~m}, \theta_{0}=45^{\circ}$, and $h=0.01 \mathrm{~m}$; for conical shell, $E=70 \mathrm{GPa}, \mu=0.3, \rho=2700 \mathrm{~kg} / \mathrm{m}^{3}$, $R_{0}=0.34 \mathrm{~m}, L=1.14 \mathrm{~m}, \varphi=3.8^{\circ}, \theta_{0}=130^{\circ}$, and $h=$ $0.002 \mathrm{~m}$; and for spherical shell, $E=70 \mathrm{GPa}, \mu=0.3, \rho=$ $2700 \mathrm{~kg} / \mathrm{m}^{3}, R=2 \mathrm{~m}, \varphi_{0}=75.7^{\circ}, \varphi_{1}=104.3^{\circ}, \theta_{0}=28.6^{\circ}$, and $h=0.01 \mathrm{~m}$. Theoretical and experimental results reported by Bardell et al. [33], Zhao et al. [35], and Liew et al. [36] as well as those obtained from finite element analyses have also been provided for comparisons. The first nine frequencies of the open shell subjected to F-F-F-F boundary conditions are listed in Table 1. The symbols "-_" in Table 1 are missing frequencies that were not considered by Liew et al. [36].

From Table 1, we can find that the first eight frequencies converge rapidly with the increasing truncated number and a very close agreement between present solutions and referential results, although different displacement admissible functions and solution procedures were used in the literature. It should be noted that the results provide by Liew et al. [36] are based on 3D shallow shell theory. The convergence study shows the excellent efficiency of the SGM-Ritz solution.
To further validate the accuracy and reliability of current solution, more numerical examples will be presented. In each case, the convergence study is performed and, for brevity purposes, only the converged results are presented here. Since the results converge well at the truncated number $M=N=$ 14 , the following calculations will be implemented with the truncated numbers.

As mentioned earlier, in the current modeling framework, all the classical boundary conditions and their combinations can be conveniently viewed as special cases by setting the values of the linear and rotational boundary springs stiffness either equal to zero or infinitely large. Thus, the effects on the modal characteristics of the linear and rotational spring's stiffness should be investigated. For the sake of generalizing the effects, the nondimensional elastic restraint parameters $\Gamma_{u}, \Gamma_{v}, \Gamma_{w}$, and $\Gamma_{W}$, which are defined as the ratios of the corresponding spring's stiffness of $\alpha$-axial, $\beta$ axial, and $z$-axial and rotational boundary spring's stiffness to the transverse flexural stiffness $D=E h^{3} / 12\left(1-\mu^{2}\right)$, are introduced here. Since current investigations about the effects on vibration behavior of the various boundary conditions are mostly concentrated on the closed shell, the open shells, that is, cylindrical shell, conical shell, and spherical shell, are introduced here to analyze the effects on vibration behavior of the various boundary spring's stiffness in this subsection. In Figure 5, variations of the 1st-, 3rd-, and 5th-order frequency parameters $\Omega=\omega L^{2} \sqrt{\rho h / D}$ against the elastic restraint parameters $\Gamma_{\zeta}$ (where $\zeta=u, v, w$ and $W$ ) of three different open shells are depicted, respectively. The material properties for the three types of considered open shells are given as follows: $E=210 \mathrm{GPa}, \mu=0.3$, and $\rho=7800 \mathrm{~kg} / \mathrm{m}^{3}$. And the geometrical dimensions are employed as follows: for the cylindrical shell, $R=2 \mathrm{~m}, L=3 \mathrm{~m}, h=0.01 \mathrm{~m}$, and $\theta_{0}=90^{\circ}$; for the conical shell, $R_{0}=1 \mathrm{~m}, L=2 \mathrm{~m}, h=0.01 \mathrm{~m}, \varphi=45^{\circ}$, and $\theta_{0}=60^{\circ}$; for the spherical shell, $R=2 \mathrm{~m}, h=0.01 \mathrm{~m}$, $\varphi_{0}=60^{\circ}, \varphi_{1}=120^{\circ}$, and $\theta_{0}=60^{\circ}$. In Figure 5, each of the shells is supposed to be clamped at edge $\alpha=0$, free at edges $\beta=0$ and $\beta=L_{\beta}$, and elastically restrained at edge $\alpha=L_{\alpha}$ by only one group of spring components with various stiffness value (varying from $10^{-4}$ to $10^{10}$ ) and the other three groups 
TABLE 1: Comparison and convergence of the first eight natural frequencies (Hz) for three open shells with F-F-F-F boundary condition.

\begin{tabular}{|c|c|c|c|c|c|c|c|c|c|}
\hline \multirow{2}{*}{ Model } & \multirow{2}{*}{$M=N$} & \multicolumn{8}{|c|}{ Model sequence } \\
\hline & & 1 & 2 & 3 & 4 & 5 & 6 & 7 & 8 \\
\hline \multirow{10}{*}{ Cylindrical shell } & 2 & 35.77 & 38.351 & 43.957 & 48.779 & 62.233 & 63.943 & 66.038 & 76.359 \\
\hline & 4 & 16.32 & 25.170 & 28.765 & 31.603 & 35.307 & 56.076 & 60.732 & 61.657 \\
\hline & 6 & 8.071 & 22.883 & 26.809 & 30.312 & 33.721 & 54.988 & 58.716 & 61.556 \\
\hline & 8 & 7.467 & 22.336 & 26.337 & 30.125 & 33.328 & 54.699 & 58.374 & 61.541 \\
\hline & 10 & 7.379 & 22.183 & 26.207 & 30.082 & 33.215 & 54.609 & 58.288 & 61.538 \\
\hline & 12 & 7.354 & 22.133 & 26.165 & 30.068 & 33.177 & 54.578 & 58.261 & 61.537 \\
\hline & 14 & 7.345 & 22.113 & 26.148 & 30.063 & 33.162 & 54.565 & 58.250 & 61.536 \\
\hline & 16 & 7.341 & 22.105 & 26.141 & 30.061 & 33.156 & 54.560 & 58.245 & 61.535 \\
\hline & Ref. [37] & 7.225 & 22.096 & 26.179 & 30.011 & 33.105 & 54.507 & 58.182 & 61.535 \\
\hline & ANSYS [37] & 7.312 & 22.094 & 26.096 & 30.014 & 33.064 & 54.400 & 58.106 & 61.517 \\
\hline \multirow{12}{*}{ Conical shell } & 2 & 38.4 & 54.85 & 84.08 & 103.0 & 159.23 & 229.3 & 353.4 & 381.1 \\
\hline & 4 & 18.7 & 25.30 & 32.04 & 36.89 & 49.87 & 72.59 & 88.88 & 96.85 \\
\hline & 6 & 15.9 & 15.98 & 21.86 & 35.56 & 46.43 & 70.10 & 79.02 & 83.43 \\
\hline & 8 & 10.2 & 14.45 & 20.23 & 35.32 & 45.74 & 69.56 & 76.99 & 78.38 \\
\hline & 10 & 8.37 & 13.60 & 19.43 & 35.25 & 45.54 & 69.32 & 76.40 & 76.91 \\
\hline & 12 & 7.75 & 13.16 & 19.04 & 35.21 & 45.47 & 69.18 & 76.18 & 76.41 \\
\hline & 14 & 7.48 & 12.92 & 18.83 & 35.17 & 45.44 & 69.05 & 76.06 & 76.19 \\
\hline & 16 & 7.48 & 12.92 & 18.83 & 35.17 & 45.44 & 69.05 & 76.06 & 76.19 \\
\hline & Ref. [37] & 7.29 & 12.59 & 18.54 & 35.19 & 45.38 & 69.06 & 75.94 & 76.05 \\
\hline & Ref. [33] & 7.21 & 12.32 & 18.21 & 34.40 & 44.32 & 67.78 & 75.43 & 76.05 \\
\hline & Experiment [33] & 7.50 & 12.70 & 18.20 & 35.60 & 46.00 & 59.50 & 70.40 & 73.10 \\
\hline & Ref. [35] & 7.33 & 12.85 & 18.80 & 35.96 & 46.18 & 70.46 & 76.52 & 76.69 \\
\hline \multirow{10}{*}{ Spherical shell } & 2 & 93.009 & 134.18 & 136.42 & 149.49 & 176.026 & 207.42 & 244.63 & 250.30 \\
\hline & 4 & 36.887 & 53.459 & 95.559 & 97.201 & 126.99 & 182.01 & 219.35 & 225.24 \\
\hline & 6 & 34.687 & 50.676 & 92.738 & 94.323 & 125.45 & 178.61 & 217.53 & 223.42 \\
\hline & 8 & 34.462 & 50.256 & 92.318 & 93.893 & 125.19 & 178.05 & 217.20 & 223.08 \\
\hline & 10 & 34.413 & 50.156 & 92.221 & 93.792 & 125.13 & 177.92 & 217.17 & 222.99 \\
\hline & 12 & 34.398 & 50.125 & 92.191 & 93.762 & 125.11 & 177.88 & 217.09 & 222.96 \\
\hline & 14 & 34.393 & 50.113 & 92.179 & 93.750 & 125.09 & 177.86 & 217.08 & 222.95 \\
\hline & 16 & 34.390 & 50.108 & 92.175 & 93.745 & 125.09 & 177.85 & 217.07 & 222.95 \\
\hline & Ref. [37] & 33.955 & 49.944 & 91.743 & 92.975 & 124.21 & 177.05 & 216.22 & 221.60 \\
\hline & Ref. [36] & 33.460 & 48.906 & 91.297 & - & 121.80 & 173.11 & 211.57 & - \\
\hline
\end{tabular}

of spring components' stiffness value with zero. Briefly, this type of boundary condition can be denoted by C-F-E ${ }^{I}-\mathrm{F}$.

From Figure 5, it is observed that the frequency parameters $\Omega$ increase as the stiffness parameters $\Gamma_{\zeta}$ increase in the certain range. Specifically, the frequency parameters almost keep at a level when the stiffness parameters $\Gamma_{\zeta}$ of the boundary springs are smaller than $10^{-1}$. As they further increase, a distinct influence can be observed, in which the frequency parameters increase rapidly; when they are beyond $10^{8}$, the frequency parameters approach the utmost and remain unchanged. As mentioned early, it is reasonable that the classical boundary condition is simulated by setting the ratios of the corresponding spring stiffness to the flexural stiffness $D=E h^{3} / 12\left(1-\mu^{2}\right)$ to be $10^{10}$. Similarly, the ratios of the coupling spring stiffness to the flexural stiffness $D$ at the interface are set to be $10^{10}$ to satisfy the rigid connective conditions for the closed shells. From Figure 5, it can be concluded that the different restraint springs have different effects on the vibration behavior of the open shells. Comparing Figure 5(c) with 5(a) and 5(b), it can be found that the influence of spring component $k_{u}$ on the spherical shell is different from the cylindrical and conical shells, which may be because the spherical shell is doubly curved. Furthermore, from Figure 5, it can be seen that the translational spring's components have effects on the vibration behavior on the shells more than the rotational spring's component for all the three types of open shells.

3.2. Free Vibration Analysis of Open Shells. In order to study the free vibration characteristics of the present method for the unified open and closed shell formulation, the vibration analysis of the open shells, that is, open cylindrical shell, open conical shell, and open spherical shell, is focused on in this section. 

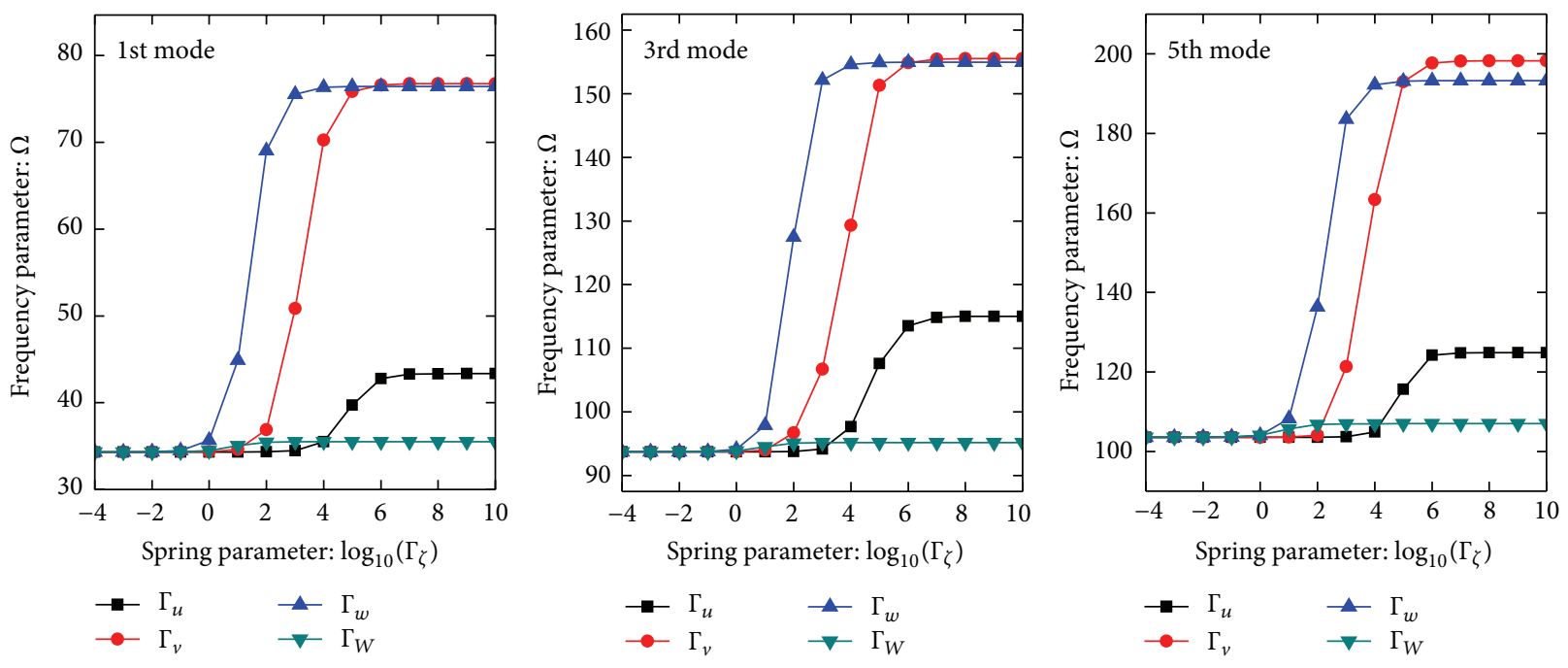

(a)
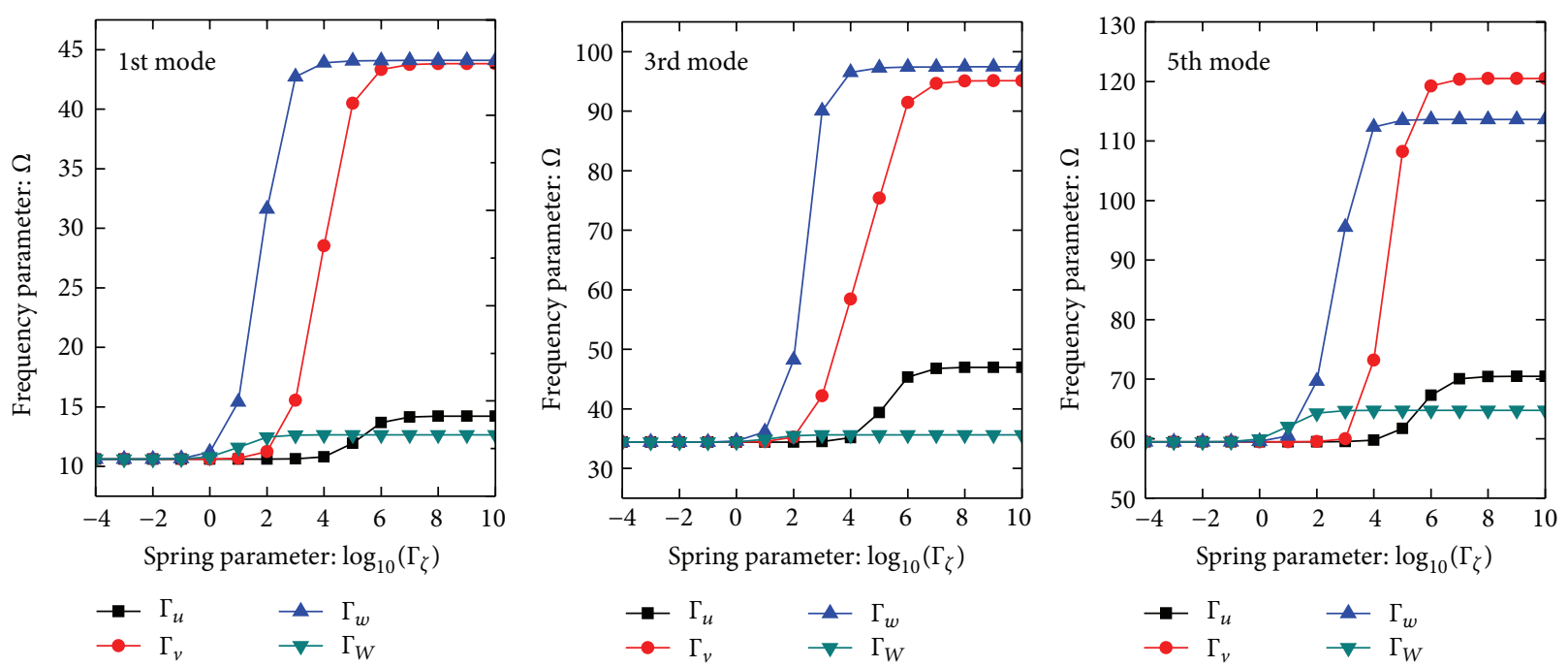

(b)
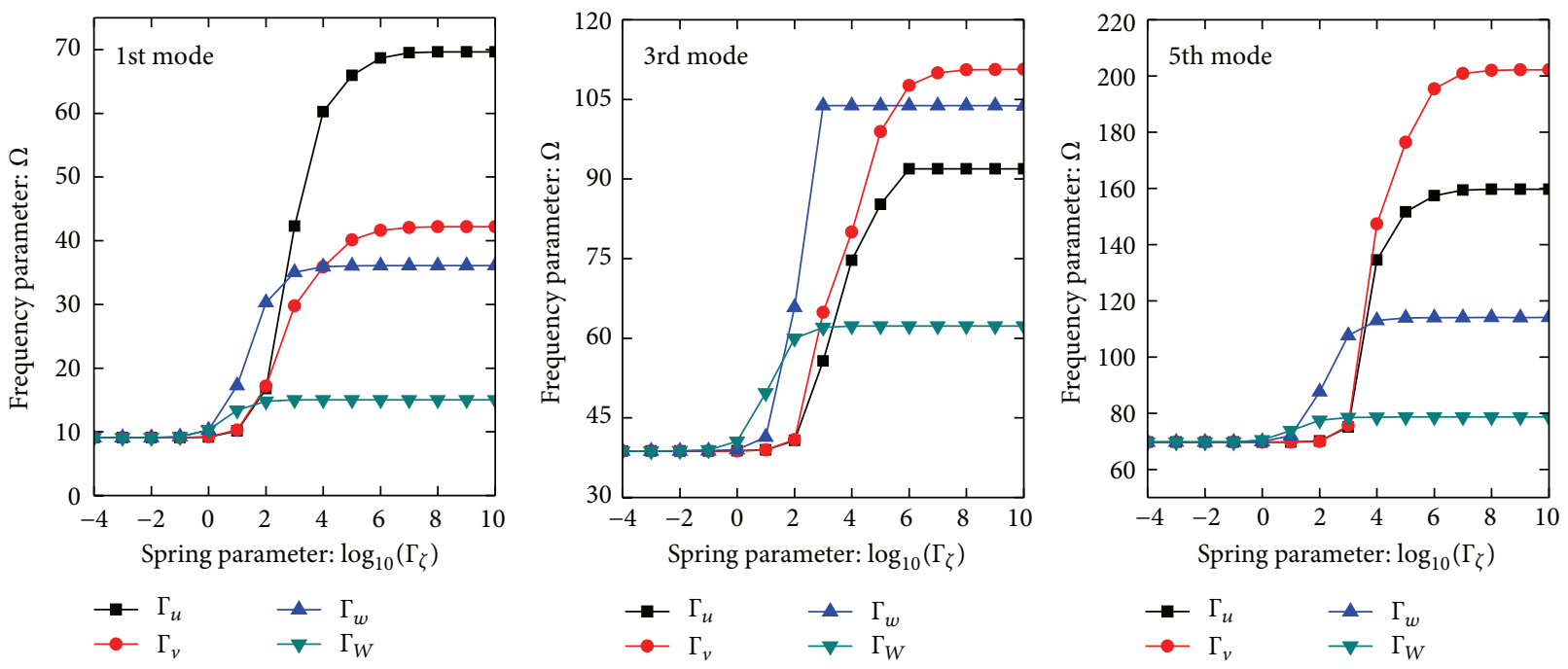

(c)

FIGURE 5: Effects of boundary spring stiffness on the 1st, 3rd, and 5th frequency parameters $\Omega=\omega L^{2} \sqrt{\rho h / D}$ of open shells with C-F-E ${ }^{I}$-F boundary conditions: (a) open cylindrical shell; (b) open conical shell; and (c) open spherical shell. 
TABLE 2: Comparison of the first four frequency parameters $\Omega=\omega L^{2} \sqrt{\rho h / D}$ for an open cylindrical shell with various boundary conditions.

\begin{tabular}{|c|c|c|c|c|c|c|c|c|}
\hline \multirow{2}{*}{ Boundary conditions } & \multicolumn{4}{|c|}{ Ref. [38] } & \multicolumn{4}{|c|}{ Present } \\
\hline & 1 & 2 & 3 & 4 & 1 & 2 & 3 & 4 \\
\hline F-F-F-F & 13.44 & 21.28 & 28.12 & 34.69 & 13.48 & 21.17 & 28.13 & 34.47 \\
\hline SD-F-F-F & 6.640 & 19.43 & 25.80 & 26.10 & 6.673 & 19.45 & 25.79 & 25.95 \\
\hline C-F-F-F & 5.164 & 8.594 & 24.65 & 28.10 & 5.180 & 8.587 & 24.60 & 28.01 \\
\hline SD-SD-F-F & 3.347 & 17.93 & 22.68 & 40.57 & 3.389 & 17.73 & 22.69 & 40.33 \\
\hline C-SD-F-F & 6.779 & 19.53 & 28.28 & 45.24 & 6.797 & 19.33 & 28.29 & 45.01 \\
\hline C-C-F-F & 8.440 & 25.57 & 29.56 & 51.11 & 8.506 & 25.42 & 29.54 & 50.90 \\
\hline SD-F-SD-F & 13.10 & 16.41 & 37.22 & 43.26 & 13.13 & 16.41 & 37.02 & 43.26 \\
\hline C-F-SD-F & 18.64 & 21.00 & 40.21 & 53.51 & 18.63 & 20.99 & 40.26 & 53.08 \\
\hline SD-SD-SD-F & 14.78 & 29.57 & 44.94 & 61.91 & 14.82 & 29.35 & 44.94 & 61.66 \\
\hline C-SD-SD-F & 19.73 & 33.15 & 54.56 & 64.20 & 19.79 & 32.96 & 54.59 & 63.93 \\
\hline C-C-SD-F & 20.08 & 39.72 & 54.05 & 74.34 & 20.54 & 39.32 & 54.88 & 74.25 \\
\hline C-F-C-F & 25.12 & 26.85 & 44.33 & 64.01 & 25.32 & 26.91 & 44.32 & 64.25 \\
\hline SD-C-SD-F & 15.79 & 36.41 & 45.24 & 64.32 & 15.84 & 36.22 & 45.24 & 64.38 \\
\hline C-SD-C-F & 25.96 & 37.66 & 65.26 & 66.90 & 26.05 & 37.53 & 65.62 & 66.80 \\
\hline C-C-C-F & 26.75 & 43.68 & 66.05 & 77.50 & 26.65 & 43.41 & 65.87 & 77.14 \\
\hline SD-SD-SD-SD & 25.48 & 49.61 & 55.84 & 80.46 & 25.32 & 49.34 & 55.71 & 80.23 \\
\hline C-SD-SD-SD & 29.05 & 52.11 & 64.19 & 87.56 & 28.90 & 51.86 & 64.08 & 87.38 \\
\hline C-C-SD-SD & 34.16 & 61.76 & 66.44 & 94.71 & 34.07 & 61.55 & 66.48 & 94.48 \\
\hline C-SD-C-SD & 33.69 & 55.30 & 73.85 & 95.62 & 33.66 & 55.13 & 74.00 & 95.75 \\
\hline C-C-C-SD & 37.38 & 63.67 & 76.00 & 99.98 & 38.05 & 64.30 & 76.14 & 102.33 \\
\hline $\mathrm{C}-\mathrm{C}-\mathrm{C}-\mathrm{C}$ & 46.08 & 74.02 & 78.10 & 109.68 & 46.14 & 74.11 & 79.14 & 109.95 \\
\hline
\end{tabular}

TABLE 3: Comparison of the first seven natural frequencies $(\mathrm{Hz})$ for an open cylindrical shell with various boundary conditions.

\begin{tabular}{|c|c|c|c|c|c|c|c|c|}
\hline \multirow{2}{*}{ Modes } & \multicolumn{4}{|c|}{ SD-F-SD-F } & \multicolumn{2}{|c|}{ SD-SD-SD-SD } & \multicolumn{2}{|c|}{ F-S-F-S } \\
\hline & Experiment [28] & Theory [28] & Ref. [37] & Present & Ref. [37] & Present & Ref. [37] & Present \\
\hline 1 & 300 & 286 & 285.162 & 286.012 & 1059.60 & 1059.17 & 837.437 & 837.467 \\
\hline 2 & 470 & 476 & 475.131 & 476.367 & 1693.43 & 1694.86 & 1862.92 & 1863.09 \\
\hline 3 & 850 & 819 & 817.600 & 819.124 & 2061.83 & 2061.58 & 1903.48 & 1903.04 \\
\hline 4 & 870 & 859 & 857.365 & 859.198 & 2068.16 & 2068.64 & 1928.89 & 1929.87 \\
\hline 5 & 1330 & 1341 & 1339.58 & 952.465 & 2401.35 & 2400.62 & 2436.56 & 2435.24 \\
\hline 6 & 1460 & 1440 & 1437.56 & 1341.65 & 3089.38 & 3088.82 & 3041.67 & 3042.74 \\
\hline 7 & 1490 & 1486 & 1486.98 & 1440.09 & 3412.99 & 3415.73 & 3351.38 & 3350.37 \\
\hline
\end{tabular}

3.2.1. Cylindrical Shells. In this section, open cylindrical shells subjected to different boundary conditions with various geometrical and material parameters are investigated. First, the accuracy and reliability of the present method for the unified open and closed shell formulation applied to an open cylindrical shell are studied. Table 2 lists the first four natural frequency parameters $\Omega=\omega L^{2} \sqrt{\rho h / D}$ obtained by present method with literature [38]. The material properties and geometrical dimensions of the open cylindrical shell are $E=$ $210 \mathrm{GPa}, \mu=0.3, \rho=7800 \mathrm{~kg} / \mathrm{m}^{3}, R=2 \mathrm{~m}, L=1 \mathrm{~m}$, $h=0.05 \mathrm{~m}$, and $\theta_{0}=28.6^{\circ}$. Table 3 compares the first seven natural frequencies calculated by present method with those in literature $[28,37]$. The material properties of the open cylindrical shell keep the same with the case in Table 2 and geometrical dimensions are $R=0.1 \mathrm{~m}, L=0.2 \mathrm{~m}$, $h=0.001 \mathrm{~m}$, and $\theta_{0}=60^{\circ}$. In order to further validate the accuracy of the present method, Table 4 compares the first five frequency parameters $\Omega=\omega R \sqrt{\rho / E}$ of the open cylindrical shell obtained by present method with those in literature [29, 37] with various geometric parameters, for example, $L / R$ and $\theta_{0}$, subjected to C-F-C-F boundary condition. The material properties remain unchanged and geometrical dimensions of the open cylindrical shell are $R=2 \mathrm{~m}$ and $h=0.02 \mathrm{~m}$. From Tables 2-4, it can be found clearly that a consistent agreement of present results and referential data is seen. The discrepancy is very small although different shell theories are used in the literature. The small discrepancy in the results may be attributed to the different solution approaches used in the literature.

As mentioned early, the unified shell formulation can deal with the free vibration analysis with arbitrary boundary condition. The accuracy and reliability of this method applied to open cylindrical shell with classical boundary condition are validated in Tables $2-4$. In the following discussion, 
TABLE 4: Comparison of the first five nondimensional frequency parameters $\Omega=\omega R \sqrt{\rho / E}$ for an open cylindrical shell with C-F-C-F boundary condition.

\begin{tabular}{ccccccccccc}
\hline$\theta_{0}$ & Modes & & \multicolumn{3}{c}{$L / R=1$} & & & \multicolumn{2}{c}{$L / R=2$} \\
& & Ref. [29] & Ref. [37] & Present & Ref. [29] & Ref. [37] & Present & Ref. [29] & $\begin{array}{c}\text { Ref. [37] } \\
\text { Present }\end{array}$ \\
\hline & 1 & 0.120 & 0.120 & 0.120 & 0.046 & 0.046 & 0.046 & 0.025 & 0.025 & 0.025 \\
$30^{\circ}$ & 2 & 0.169 & 0.169 & 0.169 & 0.054 & 0.054 & 0.054 & 0.028 & 0.028 & 0.028 \\
& 3 & 0.281 & 0.280 & 0.281 & 0.100 & 0.101 & 0.101 & 0.059 & 0.059 & 0.059 \\
& 4 & 0.330 & 0.327 & 0.331 & 0.132 & 0.132 & 0.132 & 0.066 & 0.066 & 0.066 \\
& 5 & 0.338 & 0.337 & 0.339 & 0.167 & 0.167 & 0.167 & 0.094 & 0.095 & 0.095 \\
\hline & 1 & 0.142 & 0.141 & 0.142 & 0.061 & 0.061 & 0.061 & 0.031 & 0.031 & 0.031 \\
$60^{\circ}$ & 2 & 0.153 & 0.151 & 0.153 & 0.068 & 0.069 & 0.069 & 0.054 & 0.054 & 0.054 \\
& 3 & 0.305 & 0.300 & 0.305 & 0.118 & 0.119 & 0.119 & 0.074 & 0.074 & 0.075 \\
& 4 & 0.305 & 0.303 & 0.305 & 0.128 & 0.129 & 0.129 & 0.078 & 0.078 & 0.078 \\
& 5 & 0.308 & 0.303 & 0.309 & 0.185 & 0.187 & 0.188 & 0.106 & 0.106 & 0.106 \\
\hline
\end{tabular}

TABLE 5: First six frequency parameters $\Omega=\omega L^{2} \sqrt{\rho h / D}$ for an open cylindrical shell subjected to various classical-elastic combined boundary conditions.

\begin{tabular}{lccccccccc}
\hline \multirow{2}{*}{ Modes } & \multicolumn{3}{c}{$\mathrm{E}^{1}-\mathrm{F}_{-} \mathrm{E}^{1}-\mathrm{F}$} & & \multicolumn{3}{c}{$\mathrm{E}^{2}-\mathrm{S}-\mathrm{E}^{2}-\mathrm{S}$} & \multicolumn{2}{c}{$\mathrm{E}^{3}-\mathrm{C}-\mathrm{E}^{3}-\mathrm{C}$} \\
& Ref. [37] & Present & Error (\%) & Ref. [37] & Present & Error (\%) & Ref. [37] & Present & Error (\%) \\
\hline 1 & 38.0712 & 38.1628 & 0.24050 & 128.550 & 128.4335 & 0.09062 & 156.917 & 156.882 & 0.02209 \\
2 & 51.5365 & 51.6942 & 0.30606 & 181.842 & 181.8735 & 0.01733 & 214.063 & 214.044 & 0.00899 \\
3 & 87.8899 & 87.7157 & 0.19816 & 206.415 & 206.1768 & 0.11538 & 222.556 & 222.437 & 0.05370 \\
4 & 92.1902 & 92.3438 & 0.16660 & 228.360 & 228.2684 & 0.04011 & 248.105 & 247.994 & 0.04464 \\
5 & 99.7832 & 99.9866 & 0.20384 & 279.383 & 279.053 & 0.11811 & 291.150 & 290.934 & 0.07431 \\
6 & 143.176 & 143.098 & 0.05421 & 289.155 & 288.959 & 0.06778 & 294.611 & 294.394 & 0.07367 \\
\hline
\end{tabular}

the elastically supported boundary condition is employed to further illustrate the accuracy and reliability of this method. Table 5 shows the first six frequency parameters expressed in a dimensionless form as $\Omega=\omega L^{2} \sqrt{\rho h / D}$ for an open cylindrical shell with different elastic boundary conditions. Three sets of classical-elastic combined boundary conditions are examined, namely, the $\mathrm{E}^{1}-\mathrm{F}-\mathrm{E}^{1}-\mathrm{F}, \mathrm{E}^{2}-\mathrm{S}-\mathrm{E}^{2}-\mathrm{S}$, and $\mathrm{E}^{3}$ $\mathrm{C}-\mathrm{E}^{3}-\mathrm{C}$. In comparison with the present method, results obtained by Ye et al. [37] are also included in Table 5. The material properties and geometrical dimensions used for the shells are $E=210 \mathrm{GPa}, \mu=0.3, \rho=7800 \mathrm{~kg} / \mathrm{m}^{3}, R=2 \mathrm{~m}, L=$ $3 \mathrm{~m}, h=0.05 \mathrm{~m}$, and $\theta_{0}=60^{\circ}$. In Table 5 , it can be observed that an excellent agreement can be seen from the comparison, and the difference is very small and does not exceed $0.30606 \%$ for the worst case. Moreover, some selected corresponding mode shapes for this open cylindrical shell with $\mathrm{E}^{3}-\mathrm{C}-\mathrm{E}^{3}-$ $\mathrm{C}$ boundary constraints are also depicted in Figure 6. From the competition study presented in Tables $2-5$, it can be concluded that the present method for open cylindrical shells with arbitrary boundary conditions is numerically accurate.

As mentioned earlier, the unified shell formulation can deal with vibration characteristics subjected to arbitrary boundary conditions including uniform and nonuniform boundary restraint. Some uniform boundary conditions are employed to illustrate the vibration characteristics in the examples above. It is interesting to study the free vibration of the unified shells subjected to nonuniform boundary restraint, for example, point supports, and several examples involving cylindrical shells with nonuniform boundary restraints will be discussed in following discussions. Since the vibration results for shells with point supported are very rare in literature, the first eight natural frequencies of the open cylindrical shell with multipoints clamped-supported are calculated in Table 6 as the benchmarking by researchers and reference data for practicing engineers. As a special case, the numbers and locations of the boundary point supports are limited to the equally spaced cases at boundary ends $\alpha=0$ and $\alpha=L_{\alpha}$, and the other two boundaries are free. The material properties are the same as the model in Table 5 and the geometrical dimensions are $R=0.5 \mathrm{~m}, L=1 \mathrm{~m}, h=$ $0.005 \mathrm{~m}$, and $\theta_{0}=90^{\circ}$. Furthermore, by gradually increasing the total number of the clamped points around the edge from 4 to 52 , it is found that the frequency parameters become stable when the total number of clamped points reaches 44 . In fact, these frequencies finally converge to the ones of the uniformly clamped shell case, as denoted by "C-F-C-F" in the table.

3.2.2. Conical Shells. As mentioned early, the method can obtain the vibration behavior of the open shell, for example, cylindrical shell, conical shell, and spherical shell, and the accuracy of the formulation of the open cylindrical shell by using this method has been validated. In this subsection, the accuracy of the present formulation is evaluated for the vibration problems of open conical shells with arbitrary classical boundary conditions and their combinations. For 
TABLE 6: First eight frequencies $(\mathrm{Hz})$ for an open cylindrical shell with multipoints clamped-supported on the two edges $\alpha=0$ and $\alpha=L_{\alpha}$.

\begin{tabular}{|c|c|c|c|c|c|c|c|c|}
\hline \multirow{2}{*}{$\begin{array}{l}\text { Number of } \\
\text { clamped points }\end{array}$} & \multicolumn{8}{|c|}{ Mode orders } \\
\hline & 1 & 2 & 3 & 4 & 5 & 6 & 7 & 8 \\
\hline 4 & 42.0733 & 73.7520 & 124.399 & 129.400 & 163.200 & 175.784 & 225.104 & 238.232 \\
\hline 8 & 84.2526 & 99.8218 & 184.433 & 185.978 & 234.422 & 265.143 & 295.010 & 297.424 \\
\hline 12 & 97.5774 & 106.417 & 189.546 & 194.152 & 238.902 & 274.257 & 299.952 & 302.187 \\
\hline 16 & 99.7987 & 107.456 & 136.231 & 199.570 & 249.178 & 277.450 & 308.594 & 308.774 \\
\hline 20 & 102.000 & 107.095 & 197.781 & 201.784 & 259.985 & 281.080 & 308.559 & 315.573 \\
\hline 24 & 103.398 & 109.240 & 200.089 & 204.168 & 270.959 & 283.070 & 315.387 & 317.166 \\
\hline 28 & 103.996 & 109.545 & 200.608 & 204.545 & 274.651 & 286.455 & 317.069 & 318.288 \\
\hline 32 & 104.759 & 110.067 & 201.069 & 205.168 & 276.958 & 286.988 & 317.541 & 318.674 \\
\hline 36 & 105.207 & 110.616 & 201.133 & 205.606 & 278.442 & 288.107 & 318.179 & 319.547 \\
\hline 40 & 105.194 & 111.732 & 203.235 & 206.126 & 279.873 & 287.052 & 320.491 & 321.935 \\
\hline 44 & 106.558 & 111.801 & 202.972 & 207.610 & 279.990 & 288.192 & 320.612 & 321.992 \\
\hline 48 & 106.610 & 111.865 & 203.425 & 207.548 & 280.293 & 288.029 & 321.033 & 322.303 \\
\hline 52 & 106.603 & 111.748 & 203.412 & 207.457 & 280.072 & 288.051 & 320.677 & 321.821 \\
\hline C-F-C-F & 106.596 & 111.739 & 203.401 & 207.608 & 279.849 & 287.903 & 320.618 & 321.542 \\
\hline
\end{tabular}

Open cylindrical shell with $\theta_{0}=90^{\circ}$

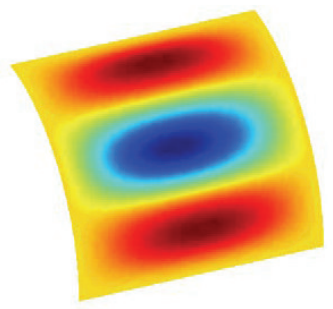

1st mode

Open cylindrical shell with $\theta_{0}=180^{\circ}$

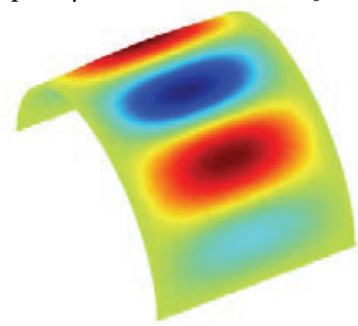

1st mode

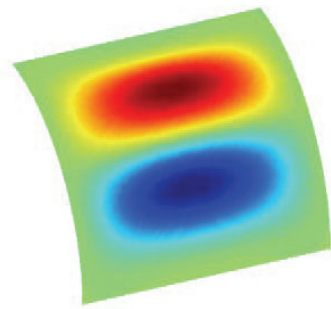

2nd mode

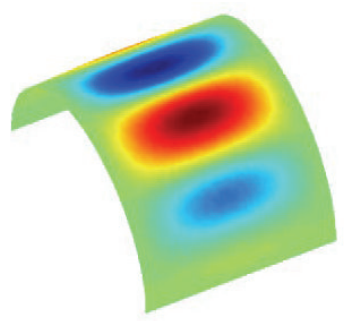

2nd mode

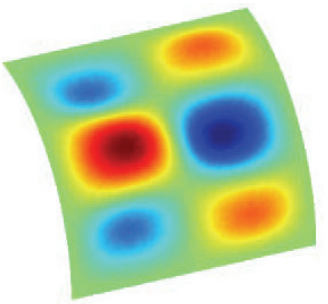

3rd mode

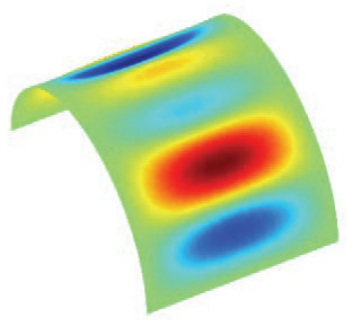

3rd mode

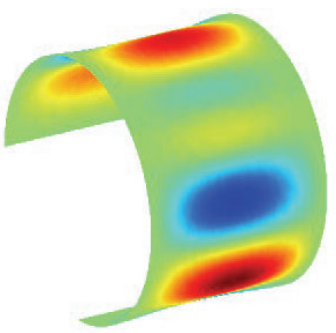

3rd mode

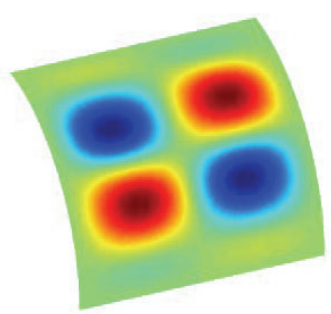

4th mode

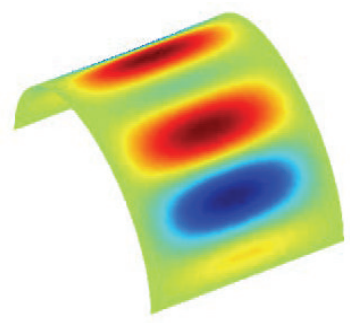

4th mode

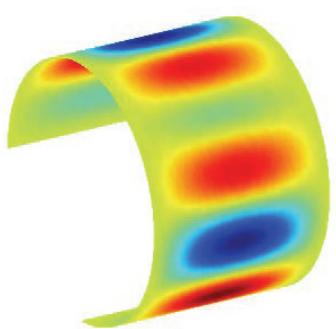

4th mode

FIGURE 6: First four mode shapes for an open cylindrical shell with $\mathrm{E}^{3}-\mathrm{C}-\mathrm{E}^{3}-\mathrm{C}$ boundary constraints. 
TABLE 7: First four frequency parameters $\Omega=\omega L^{2} \sqrt{\rho h / D}$ for an open conical shell subjected to various cone vertex angle $\varphi$ and boundary conditions.

\begin{tabular}{|c|c|c|c|c|c|c|c|c|c|}
\hline \multirow{2}{*}{$\varphi$} & \multirow{2}{*}{ Modes } & \multicolumn{2}{|c|}{ F-F-F-F } & \multicolumn{2}{|c|}{ SD-F-SD-F } & \multicolumn{2}{|c|}{ C-SD-C-SD } & \multicolumn{2}{|c|}{ C-C-C-C } \\
\hline & & Ye et al. [37] & Present & Ye et al. [37] & Present & Ye et al. [37] & Present & Ye et al. [37] & Present \\
\hline \multirow{4}{*}{$15^{\circ}$} & 1 & 21.7369 & 21.7869 & 68.3314 & 68.4257 & 276.046 & 276.156 & 362.599 & 362.681 \\
\hline & 2 & 33.2499 & 33.3564 & 80.3334 & 80.3412 & 331.696 & 331.755 & 376.082 & 376.311 \\
\hline & 3 & 66.3301 & 66.4411 & 95.9809 & 96.6836 & 377.556 & 377.743 & 517.224 & 516.001 \\
\hline & 4 & 85.5839 & 85.5869 & 158.287 & 158.323 & 445.588 & 445.206 & 540.634 & 540.451 \\
\hline \multirow{4}{*}{$30^{\circ}$} & 1 & 19.7435 & 19.8247 & 61.2279 & 61.2920 & 246.813 & 246.896 & 289.007 & 289.076 \\
\hline & 2 & 20.1969 & 20.2408 & 66.1966 & 66.2187 & 250.462 & 250.502 & 297.547 & 297.489 \\
\hline & 3 & 49.1755 & 49.1780 & 134.671 & 134.7452 & 303.748 & 303.453 & 381.886 & 379.499 \\
\hline & 4 & 57.1880 & 57.2699 & 140.764 & 140.8377 & 362.315 & 362.345 & 412.226 & 407.261 \\
\hline \multirow{4}{*}{$45^{\circ}$} & 1 & 14.1233 & 14.1762 & 53.0474 & 53.0949 & 203.828 & 203.843 & 233.139 & 233.132 \\
\hline & 2 & 19.4793 & 19.5093 & 55.4028 & 55.4244 & 212.084 & 212.089 & 239.744 & 239.578 \\
\hline & 3 & 34.3252 & 34.3282 & 114.218 & 114.295 & 234.695 & 234.516 & 303.960 & 301.209 \\
\hline & 4 & 51.7167 & 51.7673 & 121.476 & 121.563 & 282.851 & 280.819 & 315.028 & 309.892 \\
\hline \multirow{4}{*}{$60^{\circ}$} & 1 & 11.4157 & 11.4424 & 43.3049 & 149.211 & 161.854 & 161.862 & 182.496 & 182.453 \\
\hline & 2 & 18.9259 & 18.9409 & 45.8304 & 151.341 & 163.326 & 163.369 & 186.331 & 186.280 \\
\hline & 3 & 27.0994 & 27.1017 & 95.1813 & 173.651 & 186.638 & 186.534 & 232.598 & 231.343 \\
\hline & 4 & 46.9403 & 46.9606 & 102.109 & 177.658 & 225.308 & 223.702 & 247.410 & 243.849 \\
\hline \multirow{4}{*}{$75^{\circ}$} & 1 & 10.0080 & 10.0154 & 31.6590 & 31.6748 & 105.540 & 105.5392 & 125.150 & 125.101 \\
\hline & 2 & 18.0186 & 18.0226 & 34.6073 & 34.6197 & 119.710 & 119.7116 & 129.281 & 129.276 \\
\hline & 3 & 23.3268 & 23.3277 & 73.3475 & 73.3749 & 125.399 & 125.4059 & 169.937 & 169.771 \\
\hline & 4 & 40.9675 & 40.9723 & 76.1915 & 76.2144 & 148.330 & 148.2946 & 176.065 & 175.723 \\
\hline
\end{tabular}

TABLE 8: Comparison of the first six frequency parameters $\Omega=\omega L^{2} \sqrt{\rho h / D}$ for an open conical shell with various boundary conditions.

\begin{tabular}{|c|c|c|c|c|c|c|c|c|}
\hline \multirow{2}{*}{ Modes } & \multicolumn{4}{|c|}{ S-S-S-S } & \multicolumn{4}{|c|}{ C-C-C-C } \\
\hline & $h-p$ FEM [33] & ANSYS [33] & Ye et al. [37] & Present & $h-p$ FEM [33] & ANSYS [33] & Ye et al. [37] & Present \\
\hline 1 & 19.2 & 19.1 & 19.100 & 19.09991 & 35.2 & 35.0 & 35.08838 & 35.09070 \\
\hline 2 & 43.6 & 43.5 & 43.632 & 43.63151 & 66.3 & 66.0 & 6.20422 & 66.20527 \\
\hline 3 & 50.9 & 50.7 & 50.811 & 50.81127 & 74.9 & 74.4 & 74.60812 & 74.60727 \\
\hline 4 & 78.9 & 78.6 & 78.942 & 78.94172 & 108.4 & 107.7 & 108.2133 & 108.2071 \\
\hline 5 & 83.3 & 82.9 & 83.176 & 83.17624 & 113.2 & 112.2 & 112.8819 & 112.9004 \\
\hline 6 & 101.1 & 100.6 & 100.795 & 100.7949 & 134.9 & 133.0 & 133.4319 & 133.4149 \\
\hline
\end{tabular}

comparing the present results with those in literature, the material parameters used are $E=70 \mathrm{GPa}, \mu=0.3$, and $\rho=$ $2700 \mathrm{~kg} / \mathrm{m}^{3}$. And a dimensionless frequency parameter $\Omega=$ $\omega L^{2} \sqrt{\rho h / D}$ is introduced in the calculations in this subsection. Table 7 shows the comparison of the first four frequency parameters $\Omega$ for an open conical shell with various boundary conditions and cone vertex angles. The geometric dimensions of the shell are $R_{0}=1 \mathrm{~m}, L=3 \mathrm{~m}, h=0.01 \mathrm{~m}$, and $\theta_{0}=90^{\circ}$. What is more, the present formulation can also be applied to an annular plate, which involves the following procedure: setting the cone vertex angle to $90^{\circ}$. And then a conical shell can degenerate to an annular sector plate. For the sake of completeness, Table 8 compares the vibration solutions by present method of the annular sector plate, which can be treated as a special case of open conical shells, with those in literature. The material parameters remain unchanged and the geometric dimensions of the shell are $R_{0}=1 \mathrm{~m}, L=2 \mathrm{~m}$, $h=0.006 \mathrm{~m}, \theta_{0}=60^{\circ}$, and $\varphi=90^{\circ}$. It can be observed from
Tables 7 and 8 that the present results match very well with those in literature. The excellent agreements of comparisons between the proposed solutions and the published results for open conical shells with various geometric dimensions and boundary conditions separately given in Tables 7-8 indicate that the present analysis is accurate.

To further validate the accuracy and reliability of the present solutions, more complex numerical examples are presented. The vibration analysis for the open conical shell subjected to classical-elastic combined restraints is concentrated on. Table 9 compares the first six frequency parameters $\Omega=\omega L^{2} \sqrt{\rho h / D}$ with those calculated by Ye et al. [37] for the conical shell subjected to various elastically supported restraint conditions. The material properties and geometrical dimensions are employed as $E=210 \mathrm{GPa}, \mu=0.3, \rho=$ $7800 \mathrm{~kg} / \mathrm{m}^{3}, R_{0}=1 \mathrm{~m}, L=2 \mathrm{~m}, h=0.01 \mathrm{~m}, \varphi=$ $30^{\circ}$, and $\theta_{0}=90^{\circ}$. From Table 9 , it can be found that the maximum difference between present results and those 
TABLE 9: First six frequency parameters $\Omega=\omega L^{2} \sqrt{\rho h / D}$ for an open conical shell subjected to various classical-elastic combined boundary conditions.

\begin{tabular}{|c|c|c|c|c|c|c|c|c|c|}
\hline \multirow{2}{*}{ Modes } & \multicolumn{3}{|c|}{$E^{1}-F-E^{1}-F$} & \multicolumn{3}{|c|}{$E^{2}-F-E^{2}-F$} & \multicolumn{3}{|c|}{$E^{3}-F-E^{3}-F$} \\
\hline & Ref. [37] & Present & Error (\%) & Ref. [37] & Present & Error (\%) & Ref. [37] & Present & Error (\%) \\
\hline 1 & 56.9432 & 56.8022 & 0.24763 & 68.0095 & 67.9740 & 0.05226 & 68.3529 & 68.3048 & 0.07040 \\
\hline 2 & 57.1139 & 56.9227 & 0.33470 & 69.3596 & 69.3364 & 0.03347 & 69.9152 & 69.8886 & 0.03800 \\
\hline 3 & 118.408 & 118.226 & 0.15366 & 130.858 & 130.957 & 0.07561 & 131.411 & 131.269 & 0.10843 \\
\hline 4 & 125.462 & 124.511 & 0.75773 & 131.270 & 131.359 & 0.06747 & 131.693 & 131.559 & 0.10175 \\
\hline 5 & 137.038 & 136.743 & 0.21519 & 158.506 & 158.160 & 0.21803 & 164.337 & 164.089 & 0.15109 \\
\hline 6 & 153.413 & 152.120 & 0.84285 & 178.341 & 177.884 & 0.25638 & 181.088 & 180.727 & 0.19913 \\
\hline
\end{tabular}

TABLE 10: First eight frequencies (Hz) for an open conical shell with multipoints clamped-supported at the edges $\alpha=0$ and $\alpha=L_{\alpha}$.

\begin{tabular}{|c|c|c|c|c|c|c|c|c|}
\hline \multirow{2}{*}{$\begin{array}{l}\text { Number of } \\
\text { clamped points }\end{array}$} & \multicolumn{8}{|c|}{ Mode orders } \\
\hline & 1 & 2 & 3 & 4 & 5 & 6 & 7 & 8 \\
\hline 4 & 11.8729 & 20.0077 & 32.5122 & 39.5461 & 53.0354 & 55.5817 & 70.2857 & 73.8902 \\
\hline 8 & 36.7021 & 37.0652 & 68.2869 & 71.6845 & 84.1112 & 90.5067 & 96.5518 & 102.390 \\
\hline 12 & 38.0459 & 39.4913 & 74.2440 & 75.3170 & 92.7235 & 107.683 & 114.175 & 123.842 \\
\hline 16 & 40.6246 & 41.8082 & 76.1112 & 76.4630 & 96.2314 & 108.537 & 119.440 & 124.762 \\
\hline 20 & 41.5237 & 42.8696 & 78.5924 & 79.2059 & 98.5312 & 109.629 & 124.270 & 127.001 \\
\hline 24 & 41.9268 & 42.9051 & 80.1504 & 80.3934 & 101.246 & 112.015 & 128.930 & 129.004 \\
\hline 28 & 42.0010 & 42.9886 & 81.3801 & 81.3833 & 102.009 & 112.602 & 130.785 & 130.966 \\
\hline 32 & 42.2120 & 43.2225 & 81.9266 & 81.9334 & 102.514 & 113.002 & 131.522 & 131.946 \\
\hline 36 & 42.6656 & 43.7936 & 82.5368 & 83.2035 & 103.144 & 113.490 & 132.629 & 132.804 \\
\hline 40 & 42.8653 & 43.8284 & 82.6513 & 82.8204 & 103.221 & 113.492 & 133.088 & 133.376 \\
\hline 44 & 42.8620 & 43.8322 & 82.6605 & 82.8006 & 103.223 & 113.500 & 133.119 & 133.339 \\
\hline 48 & 42.8487 & 43.8083 & 82.6437 & 82.8098 & 103.261 & 113.488 & 133.307 & 133.339 \\
\hline 52 & 42.8579 & 43.8247 & 82.6678 & 82.8223 & 103.210 & 113.487 & 133.093 & 133.410 \\
\hline C-F-C-F & 42.8587 & 43.8284 & 82.6528 & 82.8179 & 103.219 & 113.493 & 133.132 & 133.349 \\
\hline
\end{tabular}

in literature is only $0.84285 \%$ for the worst case. And the present solutions match well with the results in literature, which further validate the accuracy and reliability of the current solutions. Moreover, some selected corresponding mode shapes subjected to $\mathrm{E}^{3}-\mathrm{C}-\mathrm{E}^{3}-\mathrm{C}$ boundary conditions with various subtended angles $\theta_{0}$ are shown in Figure 7.

As mentioned previously, the present method is convenient to handle the problem with nonuniform boundary restraints. Thus, a typical case under multipoints supported boundary conditions, in which the locations of the boundary point supports are equally spaced at boundary ends $\alpha=$ 0 and $\alpha=L_{\alpha}$ and the other two boundaries are free, is considered here. Table 10 shows the first eight frequencies of the open conical shells with the multipoints clampedrestrained. For simplicity and convenience, the material properties and geometry dimensions used for the shells are the same as the models used in Table 9. Similar to the open cylindrical shell with multipoints supported, the frequencies converge to the ones of the uniformly clamped shell case, as denoted by "C-F-C-F" in the table, when the total numbers of clamped points reaches 40 .

3.2.3. Spherical Shells. The free vibration analysis for the open cylindrical and conical shells has been studied and the accuracy of the present method is validated well. In order to further investigate the accuracy of the unified formulation for the open shell, in this subsection, the present formulation is applied to study the free vibrations of open spherical shells with arbitrary boundaries for which very limited amount of vibration results is available in the literature. In Table 11, comparison solutions by present method and the reported literature [38] of the first four nondimensional frequency parameters $\Omega=\omega L^{2} \sqrt{\rho h / D}$ for an open spherical shell with various boundary conditions are presented. The material properties and geometrical dimensions used for the spherical shell are given as follows: $E=70 \mathrm{GPa}, \mu=0.3, \rho=$ $2700 \mathrm{~kg} / \mathrm{m}^{3}, R=5 \mathrm{~m}, h=0.05 \mathrm{~m}, \varphi_{0}=84.3^{\circ}, \varphi_{1}=$ $95.7^{\circ}$, and $\theta_{0}=11.5^{\circ}$. Having gained confidence in present method, some further numerical results for open spherical shells with several types of classical boundary conditions and their combinations are given in the following presentation. In Table 12, the comparison of the first four nondimension frequency parameters $\Omega=\omega L^{2} \sqrt{\rho h / D}$ for an open spherical shell with material and geometric parameters, $E=210 \mathrm{GPa}$, $\mu=0.3, \rho=7800 \mathrm{~kg} / \mathrm{m}^{3}, R=2 \mathrm{~m}, h=0.01 \mathrm{~m}, \varphi_{0}=45^{\circ}$, $\varphi_{1}=135^{\circ}$, and $\theta_{0}=90^{\circ}$, subjected to various boundary conditions, is presented. The comparison is performed with numerical solutions by Ye et al. [37]. From Tables 11 and 


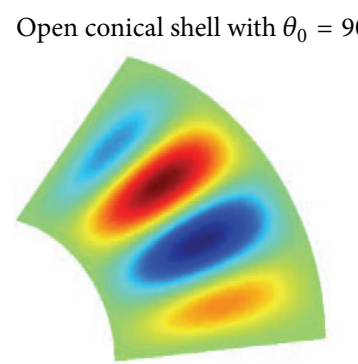

1st mode

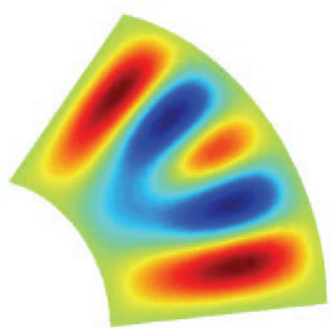

3rd mode

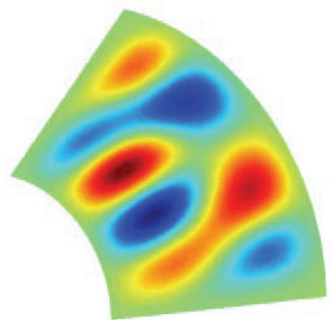

5 th mode

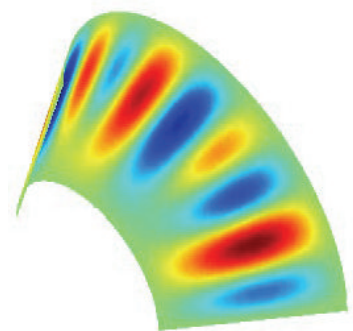

5 th mode

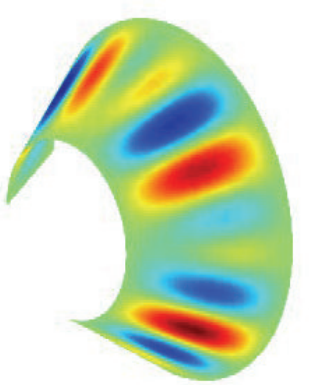

5 th mode

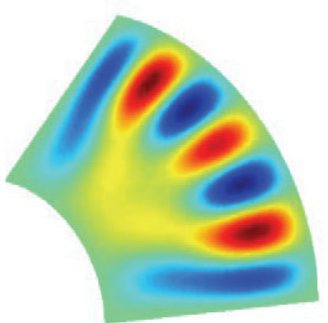

7th mode

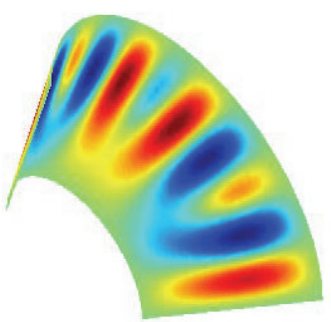

7th mode

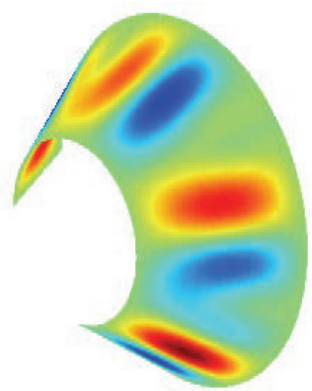

7th mode

FIGURE 7: Some selected mode shapes for an open conical shell with $\mathrm{E}^{3}-\mathrm{C}-\mathrm{E}^{3}-\mathrm{C}$ boundary conditions.

12 , it can be obviously found that the results calculated by present method match well with the corresponding solutions in literatures $[37,38]$. The excellent agreement results for the open spherical shell can further illustrate the accuracy of the unified shell formulation.

Although all the above examples fall into the category of the classical homogeneous boundary conditions, it has been demonstrated that they can be solved universally under the current framework by simply modifying the stiffness of the corresponding elastic springs. Since vibration results for the spherical shells with arbitrary boundary conditions are rare in the literature, some further numerical results for an open spherical shell with different elastically supported boundary conditions and their combinations are given in Table 13, and first eight corresponding mode shapes with $\mathrm{E}^{1}-\mathrm{C}-\mathrm{E}^{1}-\mathrm{C}$ boundary conditions are also drawn in Figure 8. A more complicated problem is one in which the spherical shell is multipoints clamped-constrained at boundary ends and the locations of these boundary point supports are equally spaced at boundaries $\alpha=0$ and $\alpha=L_{\alpha}$. Table 14 shows the first eight frequencies with the various numbers of the clamped points. The material properties and geometrical dimensions in Tables 13 and 14 are the same as the model in Table 12. From Table 13, it can be seen that the frequency parameter increases with the boundary conditions changing from $\Lambda-F-\Lambda-F$ to $\Lambda$-C- $\Lambda-\mathrm{C}\left(\Lambda=\mathrm{E}^{1}, \mathrm{E}^{2}\right.$ and $\left.\mathrm{E}^{3}\right)$, which is due to the increasing boundary spring stiffness. Furthermore, a slight increasing frequency parameter can be observed with the boundary conditions varying from $\Lambda$-S- $\Lambda$-S to $\Lambda$-C- $\Lambda$-C while a huge increasing value is found subjected to the boundary restraints changing from $\Lambda$-F- $\Lambda$-F to $\Lambda$-S- $\Lambda$-S. From Table 14, it is found that the frequency becomes stable when the total number of clamped points reaches 40 and these frequencies finally converge to the ones of the uniformly C-F-C-F shell case.

3.3. Free Vibration Analysis of Closed Shells. As mentioned earlier, the unified formulation can be applied to open and closed shell structure. In Section 3.2, the accuracy of the formulation applied to open shell has been verified. In order to further validate the accuracy of the unified method for the closed shells, the free vibration analysis of the closed shells, for example, the closed cylindrical shell, conical shell, 
TABLE 11: Comparison of the first four frequency parameters $\Omega=\omega L^{2} \sqrt{\rho h / D}$ for an open spherical shell subjected to various boundary conditions.

\begin{tabular}{|c|c|c|c|c|c|c|c|c|}
\hline \multirow{2}{*}{$\begin{array}{l}\text { Boundary } \\
\text { conditions }\end{array}$} & \multicolumn{4}{|c|}{ Ref. [38] } & \multicolumn{4}{|c|}{ Present } \\
\hline & 1 & 2 & 3 & 4 & 1 & 2 & 3 & 4 \\
\hline F-F-F-F & 13.46 & 19.56 & 25.99 & 34.85 & 13.40 & 19.44 & 25.77 & 34.59 \\
\hline SD-F-F-F & 6.629 & 15.34 & 25.40 & 27.08 & 6.582 & 15.32 & 25.28 & 26.76 \\
\hline C-F-F-F & 3.754 & 8.492 & 21.53 & 28.26 & 4.060 & 8.702 & 21.58 & 27.95 \\
\hline SD-SD-F-F & 3.367 & 17.32 & 20.79 & 39.71 & 3.340 & 17.20 & 20.61 & 39.40 \\
\hline C-SD-F-F & 5.566 & 19.35 & 25.47 & 44.64 & 5.753 & 19.19 & 25.38 & 44.47 \\
\hline C-C-F-F & 7.894 & 23.90 & 27.92 & 49.27 & 8.172 & 23.79 & 27.75 & 49.02 \\
\hline SD-F-SD-F & 10.07 & 16.10 & 38.89 & 39.59 & 10.03 & 15.98 & 38.43 & 39.54 \\
\hline C-F-SD-F & 15.63 & 21.68 & 41.84 & 49.95 & 15.76 & 24.71 & 41.48 & 49.83 \\
\hline SD-SD-SD-F & 12.22 & 30.50 & 41.85 & 60.14 & 12.15 & 30.15 & 41.77 & 59.80 \\
\hline C-SD-SD-F & 18.01 & 33.76 & 52.05 & 65.24 & 18.13 & 33.56 & 51.89 & 64.57 \\
\hline C-C-SD-F & 18.68 & 38.58 & 52.19 & 72.13 & 18.69 & 38.35 & 52.24 & 71.80 \\
\hline C-F-C-F & 25.30 & 28.67 & 45.53 & 61.34 & 26.06 & 30.82 & 45.31 & 61.20 \\
\hline SD-C-SD-F & 12.95 & 35.75 & 42.26 & 64.09 & 12.86 & 35.32 & 42.17 & 63.59 \\
\hline C-SD-C-F & 26.34 & 38.07 & 63.08 & 68.01 & 27.07 & 38.02 & 63.23 & 67.31 \\
\hline C-C-C-F & 26.62 & 42.71 & 63.56 & 77.64 & 27.68 & 42.53 & 63.53 & 76.98 \\
\hline SD-SD-SD-SD & 23.70 & 51.04 & 51.04 & 80.02 & 23.45 & 50.33 & 50.82 & 79.36 \\
\hline C-SD-SD-SD & 27.32 & 53.32 & 60.17 & 87.13 & 27.23 & 52.72 & 60.00 & 86.59 \\
\hline C-C-SD-SD & 30.64 & 61.88 & 62.36 & 93.66 & 30.51 & 61.36 & 62.02 & 93.30 \\
\hline C-SD-C-SD & 32.47 & 55.41 & 70.37 & 95.06 & 32.60 & 55.78 & 70.43 & 95.00 \\
\hline C-C-C-SD & 35.51 & 64.76 & 72.13 & 101.40 & 35.63 & 64.13 & 72.15 & 101.09 \\
\hline C-C-C-C & 40.26 & 74.17 & 74.43 & 108.66 & 40.49 & 73.77 & 74.42 & 108.32 \\
\hline
\end{tabular}

TABLE 12: First four frequency parameters $\Omega=\omega L^{2} \sqrt{\rho h / D}$ for an open spherical shell with various boundary conditions.

\begin{tabular}{|c|c|c|c|c|c|c|c|c|}
\hline \multirow{2}{*}{ Boundary conditions } & \multicolumn{4}{|c|}{ Ref. [37] } & \multicolumn{4}{|c|}{ Present } \\
\hline & 1 & 2 & 3 & 4 & 1 & 2 & 3 & 4 \\
\hline F-F-F-S & 18.0503 & 22.6720 & 48.7319 & 65.5055 & 19.2022 & 22.7991 & 50.2213 & 66.8198 \\
\hline F-F-S-S & 45.3203 & 61.1681 & 139.886 & 155.410 & 46.8427 & 61.6499 & 142.512 & 156.769 \\
\hline F-S-S-S & 381.345 & 388.913 & 713.714 & 756.822 & 389.683 & 395.471 & 727.650 & 768.011 \\
\hline F-F-F-C & 18.8440 & 23.4206 & 49.3404 & 68.0959 & 20.0030 & 23.5524 & 50.8671 & 69.2686 \\
\hline F-F-C-C & 46.5172 & 63.1538 & 144.447 & 158.654 & 48.0751 & 63.6089 & 147.277 & 159.850 \\
\hline F-C-C-C & 396.124 & 408.000 & 756.727 & 779.639 & 403.649 & 415.983 & 771.244 & 789.339 \\
\hline S-S-S-S & 1528.82 & 1542.01 & 1548.17 & 1601.85 & 1528.90 & 1542.11 & 1548.27 & 1601.91 \\
\hline S-S-S-C & 1531.94 & 1542.28 & 1549.23 & 1603.29 & 1532.07 & 1542.39 & 1549.35 & 1603.37 \\
\hline S-S-C-C & 1534.26 & 1543.64 & 1549.31 & 1604.18 & 1534.43 & 1543.59 & 1549.43 & 1604.27 \\
\hline S-C-C-C & 1537.73 & 1543.64 & 1550.42 & 1605.67 & 1537.95 & 1543.77 & 1550.55 & 1605.78 \\
\hline C-C-C-C & 1540.15 & 1544.85 & 1550.50 & 1606.56 & 1541.18 & 1546.99 & 1553.16 & 1606.57 \\
\hline
\end{tabular}

and spherical shell, will be investigated in the following text. From the procedure of the modeling framework, it can be known that the open shell can be expanded to a closed one conveniently just by setting the value of the coupling springs to be infinitely large when the subtended angle equals $360^{\circ}$. Furthermore, it is noted that the boundary restraint spring's stiffness at the end edges $\beta=0$ and $\beta=360^{\circ}$ is automatically set to be zero. First of all, the coupling spring's effect on the vibration behavior for the shells, for example, cylindrical shell, conical shell, and spherical shell, is concentrated on. Similar to the study for the effects on vibration characteristics of the boundary restraint spring, the nondimensional coupling spring parameters $\Gamma_{u}, \Gamma_{v}, \Gamma_{w}$, and $\Gamma_{W}$, which are defined as the ratios of the corresponding translational spring's stiffness of $\alpha$-axial, $\beta$-axial, and $z$-axial and rotational coupling spring's stiffness to the transverse flexural stiffness $D=E h^{3} / 12\left(1-\mu^{2}\right)$, are employed here. In Figure 9, variation of the 1st- and 3rd-order frequency parameters $\Omega=\omega R \sqrt{\rho\left(1-\mu^{2}\right) / E}$ against the coupling spring 
TABLE 13: First eight frequency parameters $\Omega=\omega L^{2} \sqrt{\rho h / D}$ for an open spherical shell subjected to various classical-elastic combined boundary conditions.

\begin{tabular}{|c|c|c|c|c|c|c|c|c|}
\hline \multirow{2}{*}{ Boundary conditions } & \multicolumn{8}{|c|}{ Modes } \\
\hline & 1 & 2 & 3 & 4 & 5 & 6 & 7 & 8 \\
\hline$E^{1}-F-E^{1}-F$ & 139.644 & 155.540 & 177.982 & 178.238 & 296.232 & 297.075 & 376.141 & 390.785 \\
\hline$E^{2}-F-E^{2}-F$ & 171.100 & 183.000 & 189.780 & 191.112 & 337.909 & 374.572 & 386.047 & 398.328 \\
\hline$E^{3}-F-E^{3}-F$ & 51.1307 & 187.620 & 221.996 & 253.487 & 358.578 & 422.613 & 476.818 & 517.802 \\
\hline$E^{1}-S-E^{1}-S$ & 1527.08 & 1536.94 & 1545.89 & 1600.52 & 1604.08 & 1607.37 & 1614.80 & 1619.77 \\
\hline$E^{2}-S-E^{2}-S$ & 752.556 & 1379.20 & 1496.59 & 1529.73 & 1543.95 & 1568.62 & 1593.51 & 1602.16 \\
\hline$E^{3}-S-E^{3}-S$ & 1197.71 & 1534.13 & 1544.01 & 1553.96 & 1589.09 & 1604.06 & 1608.02 & 1612.91 \\
\hline$E^{1}-C-E^{1}-C$ & 1534.85 & 1537.51 & 1548.21 & 1603.92 & 1605.13 & 1612.69 & 1615.10 & 1624.01 \\
\hline$E^{2}-C-E^{2}-C$ & 1425.59 & 1513.95 & 1536.62 & 1547.13 & 1554.03 & 1599.93 & 1603.55 & 1606.38 \\
\hline$E^{3}-C-E^{3}-C$ & 1539.58 & 1546.14 & 1554.80 & 1590.30 & 1607.21 & 1612.97 & 1614.36 & 1619.72 \\
\hline
\end{tabular}

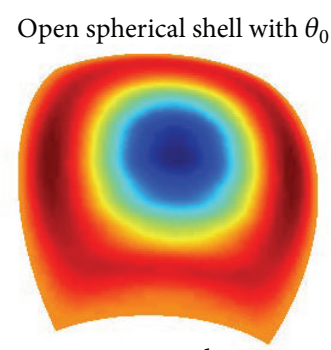

1st mode

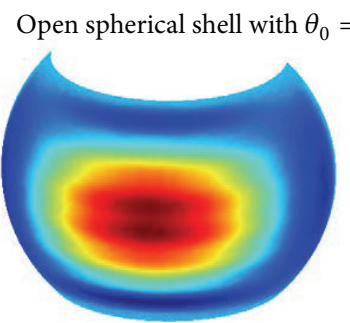

1st mode

Open spherical shell with $\theta_{0}=270^{\circ}$

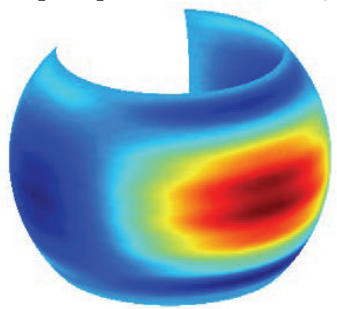

1st mode

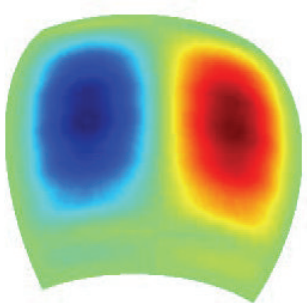

2nd mode

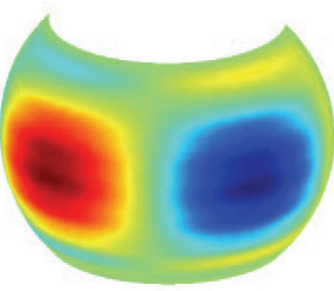

2nd mode

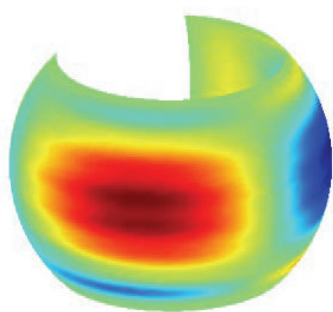

2nd mode

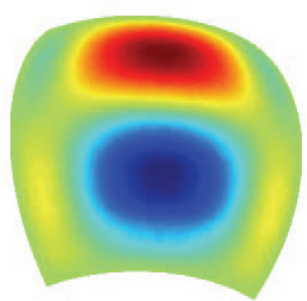

3rd mode

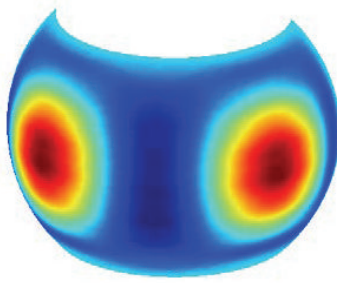

3rd mode

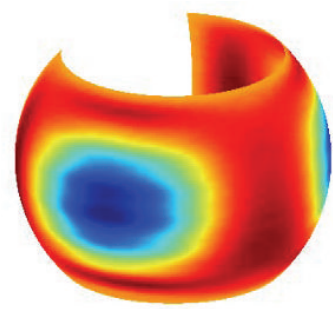

3rd mode

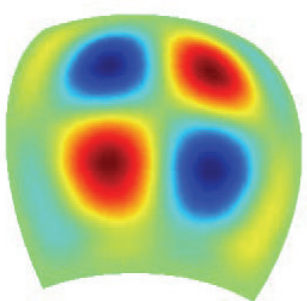

4th mode

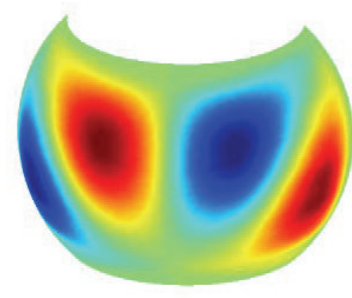

4th mode

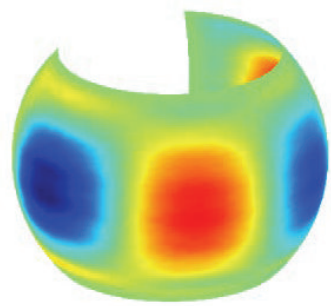

4th mode

FIGURE 8: First four mode shapes for an open spherical shell with $E^{1}-C-E^{1}-C$ boundary conditions.

parameters $\Gamma_{\zeta}$ (where $\zeta=u, v, w$ and $W$ ) of three different shells are depicted, respectively. It is noted that $R$ denotes the radius of the smaller circular edge $R_{0}$ for the conical shell. The material properties for the three types of considered shells are given as follows: $E=210 \mathrm{GPa}, \mu=0.3$, and $\rho=7800 \mathrm{~kg} / \mathrm{m}^{3}$. And the geometrical dimensions are used as follows: for the cylindrical shell, $R=2 \mathrm{~m}, L=3 \mathrm{~m}$, and $h=0.01 \mathrm{~m}$; for the conical shell, $R_{0}=1 \mathrm{~m}, L=2 \mathrm{~m}, h=0.01 \mathrm{~m}$, and $\varphi=45^{\circ}$; for the spherical shell, $R=1 \mathrm{~m}, h=0.01 \mathrm{~m}, \varphi_{0}=30^{\circ}$, and $\varphi_{1}=90^{\circ}$. In Figure 9, each of the shells is supposed to be clamped at edges $\alpha=0$ and $\alpha=L_{\alpha}$, and the junction edge is coupled with all the four groups of springs, three groups of which are with infinite stiffness $\left(10^{10} \mathrm{D}\right)$ and the remaining one is assigned at different stiffness value (varying from $10^{-4} \mathrm{D}$ to $\left.10^{10} \mathrm{D}\right)$.

From Figure 9, it can be found that the frequency parameters $\Omega$ increase as the stiffness parameters $\Gamma_{\zeta}$ increase in the certain range. Specifically, it can be found from Figure 9(a) 
TABLE 14: First eight frequencies $(\mathrm{Hz})$ for an open spherical shell with multipoints clamped-supported on the two edges $\alpha=0$ and $\alpha=L_{\alpha}$.

\begin{tabular}{|c|c|c|c|c|c|c|c|c|}
\hline \multirow{2}{*}{$\begin{array}{l}\text { Number of } \\
\text { clamped points }\end{array}$} & \multicolumn{8}{|c|}{ Mode orders } \\
\hline & 1 & 2 & 3 & 4 & 5 & 6 & 7 & 8 \\
\hline 4 & 12.2312 & 17.9155 & 19.5800 & 29.0130 & 30.5394 & 51.4391 & 51.7373 & 56.4100 \\
\hline 8 & 40.0975 & 42.7725 & 48.9036 & 53.6622 & 90.4094 & 94.7601 & 106.301 & 111.696 \\
\hline 12 & 12.3097 & 43.6488 & 52.7028 & 63.2851 & 82.3730 & 102.290 & 113.423 & 115.059 \\
\hline 16 & 28.0964 & 45.0074 & 54.4617 & 64.3798 & 90.0115 & 103.671 & 118.469 & 129.903 \\
\hline 20 & 20.5605 & 46.5769 & 55.9624 & 63.4935 & 89.0453 & 105.809 & 119.636 & 127.748 \\
\hline 24 & 10.8180 & 47.3839 & 56.3886 & 60.9230 & 87.8615 & 106.645 & 119.007 & 120.778 \\
\hline 28 & 47.4370 & 48.1909 & 56.1141 & 61.0136 & 81.8681 & 106.898 & 120.396 & 120.396 \\
\hline 32 & 47.2956 & 56.0249 & 61.1083 & 65.9971 & 78.4332 & 107.184 & 119.880 & 120.514 \\
\hline 36 & 46.9057 & 55.4473 & 61.0340 & 73.1757 & 106.791 & 107.405 & 119.547 & 120.301 \\
\hline 40 & 46.6960 & 55.3741 & 60.8831 & 72.6460 & 107.236 & 111.691 & 119.571 & 120.434 \\
\hline 44 & 47.2532 & 55.2890 & 61.1750 & 73.6090 & 105.835 & 107.287 & 119.928 & 120.366 \\
\hline 48 & 46.8644 & 55.4429 & 60.9559 & 72.8072 & 107.341 & 110.834 & 119.432 & 120.504 \\
\hline 52 & 46.8610 & 55.3493 & 61.1851 & 72.8144 & 107.345 & 111.739 & 119.527 & 120.262 \\
\hline $\mathrm{CC}$ & 46.9276 & 55.3297 & 61.3200 & 72.6933 & 107.363 & 111.138 & 119.577 & 120.454 \\
\hline
\end{tabular}

TABLE 15: Comparison of the natural frequencies for a circular cylindrical shell with C-F and C-C boundary conditions.

\begin{tabular}{|c|c|c|c|c|c|c|c|}
\hline \multicolumn{2}{|c|}{ Mode orders } & \multicolumn{3}{|c|}{ C-F } & \multicolumn{3}{|c|}{$\mathrm{C}-\mathrm{C}$} \\
\hline$m$ & $n$ & FEM [11] & Ref. [11] & Present & FEM [11] & Ref. [11] & Present \\
\hline \multirow{6}{*}{1} & 0 & 1614.9 & 1615.2 & 1615.2 & 3229.8 & 3230.3 & 3230.4 \\
\hline & 1 & 467.58 & 467.34 & 468.10 & 1882.8 & 1880.9 & 1883.5 \\
\hline & 2 & 315.33 & 316.02 & 315.30 & 899.59 & 898.18 & 900.78 \\
\hline & 3 & 768.38 & 768.45 & 768.00 & 896.97 & 896.56 & 897.71 \\
\hline & 4 & 1463.3 & 1462.8 & 1462.5 & 1501.9 & 1501.6 & 1502.0 \\
\hline & 5 & 2363.1 & 2362.2 & 2362.3 & 2386.1 & 2386.0 & 2386.3 \\
\hline \multirow{6}{*}{2} & 0 & 2583.1 & 2581.7 & 2583.8 & 5146.0 & 5139.8 & 5147.8 \\
\hline & 1 & 2044.0 & 2043.3 & 2045.1 & 3850.7 & 3848.9 & 3851.5 \\
\hline & 2 & 936.02 & 936.25 & 937.22 & 2017.8 & 2014.1 & 2019.9 \\
\hline & 3 & 914.16 & 915.13 & 914.71 & 1390.9 & 1388.9 & 1393.5 \\
\hline & 4 & 1518.7 & 1519.0 & 1518.6 & 1676.4 & 1676.0 & 1678.0 \\
\hline & 5 & 2401.9 & 2401.8 & 2401.8 & 2472.5 & 2472.6 & 2474.0 \\
\hline
\end{tabular}

that the values of the two natural frequency parameters for cylindrical shell increase rapidly in the range of $\Gamma_{\zeta}\left(10^{-2}-\right.$ $10^{7}$ ) and keep at a level outside this range. In Figure 9(b), the frequency parameters for conical shell almost remain unchanged when the stiffness of the spring parameters $\Gamma_{\zeta}$ is larger than $10^{8}$ or smaller than $10^{-2}$. In Figure 9(c), the influences of the coupling spring parameters of a spherical shell on frequency parameters are given. It is shown that the frequency curves change greatly within the spring parameters range $\left(10^{2}\right.$ to $\left.10^{7}\right)$. From Figures $9(\mathrm{a})-9(\mathrm{c})$, the frequency parameters almost keep at a level when the stiffness parameters $\Gamma_{\zeta}$ of the coupling springs are smaller than $10^{-2}$. As they further increase, a distinct influence can be observed, in which the frequency parameters increase rapidly, when they are beyond $10^{8}$, the frequency parameters approach the utmost and remain unchanged. Thus, it is reasonable that the rigid connective condition can be simulated by setting the ratios of the corresponding coupling spring stiffness to the flexural stiffness $D=E h^{3} / 12\left(1-\mu^{2}\right)$ to be $10^{10}$. In the following discussion, the value is employed to satisfy the rigid coupling condition at the interface for the shell with subtended angle $\theta_{0}=360^{\circ}$ and then the open shells are expanded to corresponding closed shells. Furthermore, comparing Figure 9(c) with 9(a) and 9(b), it can be found that the influence of spring components $k_{w}$ and $K_{w}$ on the spherical shell is different from the cylindrical and conical shells, which may be because the spherical shell is doubly curved.

3.3.1. Cylindrical Shells. First of all, the accuracy of the formulation applied to a closed cylindrical shell is validated in Table 15. The comparison is carried out with two classical boundary conditions, for example, C-F and C-C, to verify the proposed method using the following material and geometric parameters: $E=210 \mathrm{GPa}, \mu=0.28, \rho=7800 \mathrm{~kg} / \mathrm{m}^{3}$, $R=0.0635 \mathrm{~m}, L=0.502 \mathrm{~m}$, and $h=0.00163 \mathrm{~m}$. 

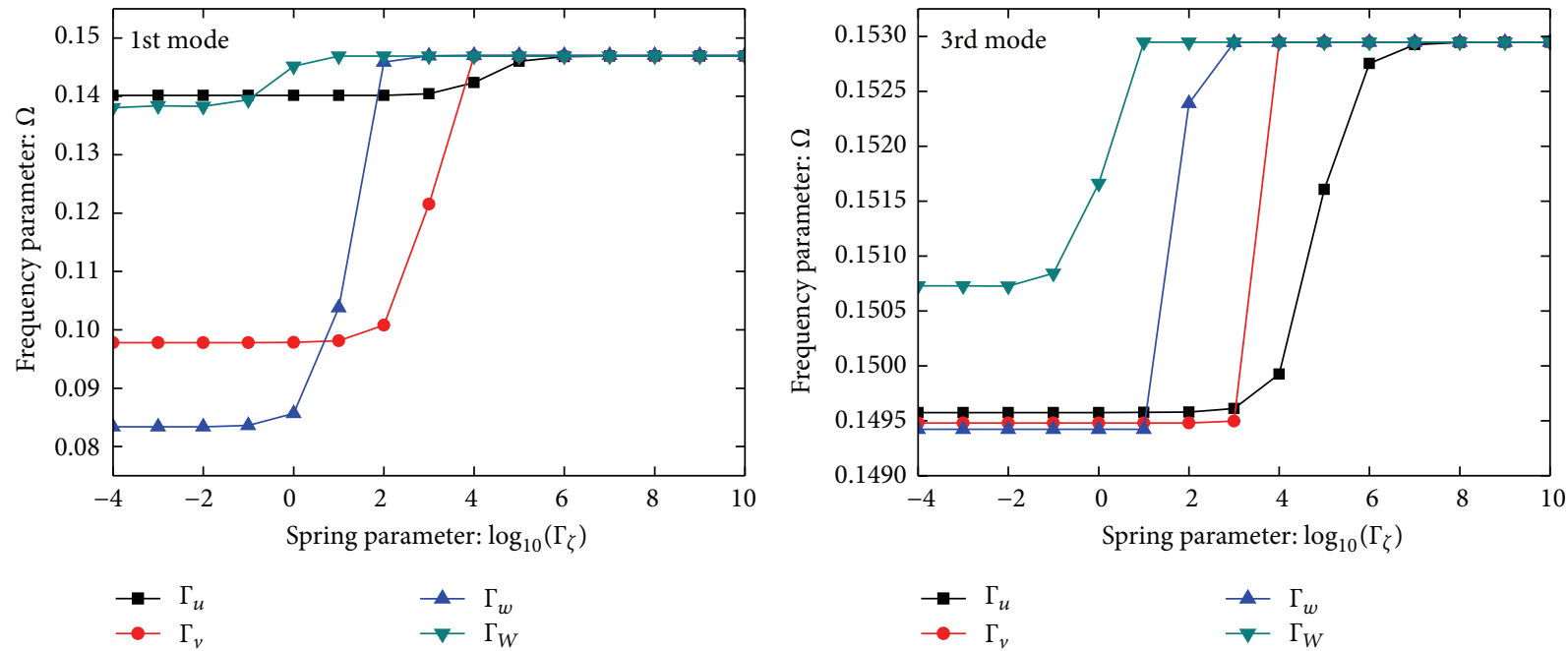

(a)
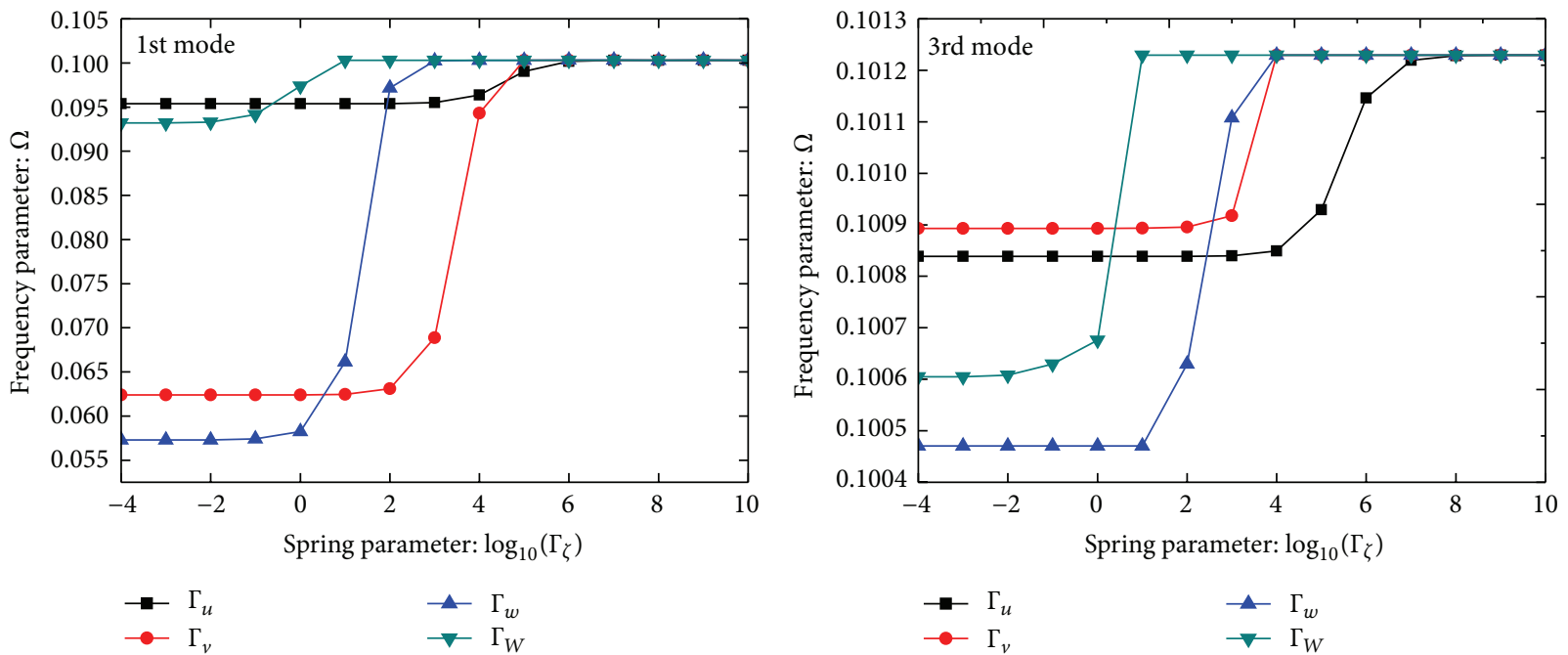

(b)
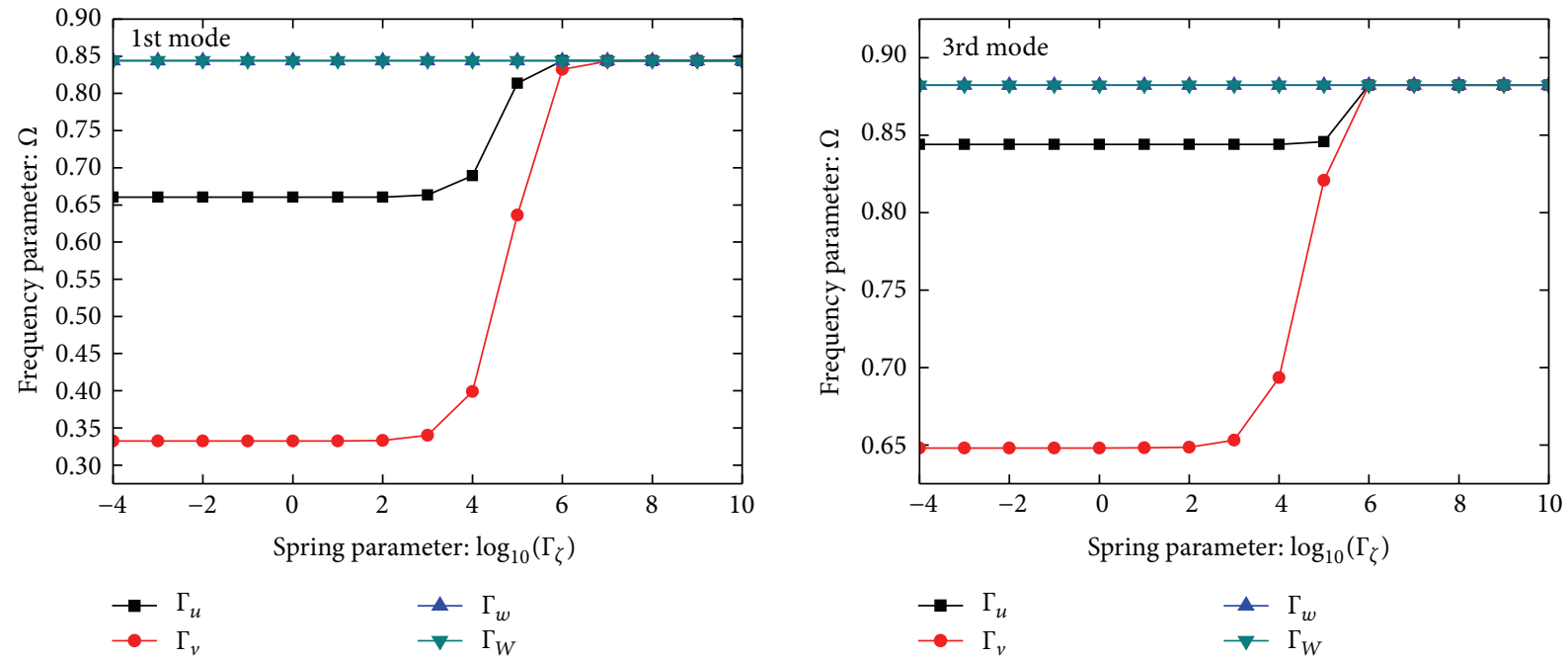

(c)

FIGURE 9: Effects of coupling spring stiffness on the 1st and 3rd frequency parameters $\Omega=\omega R \sqrt{\rho\left(1-\mu^{2}\right) / E}$ of shells with subtended angle $\theta_{0}=360^{\circ}$ subjected to C-C boundary conditions: (a) cylindrical shell, (b) conical shell, and (c) spherical shell. 
TABLE 16: First four frequencies for a clamped elastically supported shell $\left(k_{\alpha L}^{u}=k_{\alpha L}^{v}=K_{\alpha L}^{w}=0\right)$.

\begin{tabular}{|c|c|c|c|c|c|c|c|c|}
\hline \multirow{2}{*}{$\begin{array}{l}\text { Boundary } \\
\text { conditions } k_{\alpha L}^{w}\end{array}$} & \multicolumn{4}{|c|}{ Ref. [11] } & \multicolumn{4}{|c|}{ Present } \\
\hline & 1 & 2 & 3 & 4 & 1 & 2 & 3 & 4 \\
\hline 0 & 131.99 & 249.82 & 262.81 & 377.00 & 131.57 & 249.36 & 262.91 & 376.55 \\
\hline $0.01 G$ & 183.01 & 278.17 & 279.76 & 404.12 & 183.11 & 278.17 & 280.00 & 404.23 \\
\hline $0.1 G$ & 298.01 & 310.07 & 364.98 & 490.35 & 299.10 & 310.28 & 366.51 & 490.41 \\
\hline G & 315.07 & 339.86 & 473.26 & 491.64 & 315.65 & 341.04 & 478.35 & 491.70 \\
\hline $10^{6} G$ & 315.15 & 343.43 & 491.36 & 501.06 & 316.26 & 346.25 & 491.84 & 508.34 \\
\hline $10^{8} G$ & 315.15 & 343.43 & 491.36 & 501.08 & 316.25 & 346.23 & 491.84 & 508.25 \\
\hline
\end{tabular}

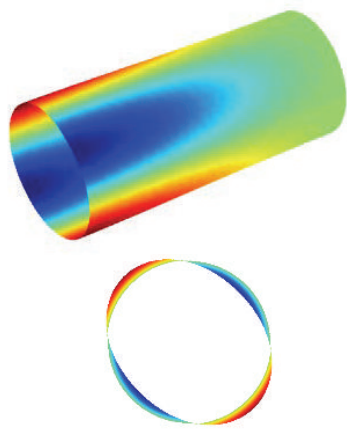

(a) 1st mode

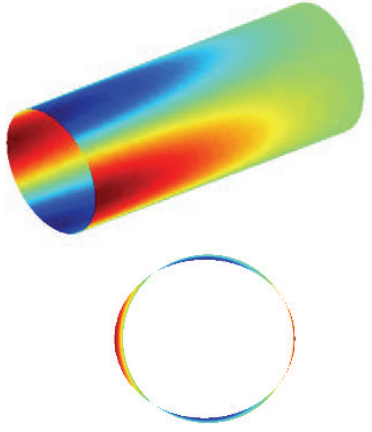

(b) 2nd mode

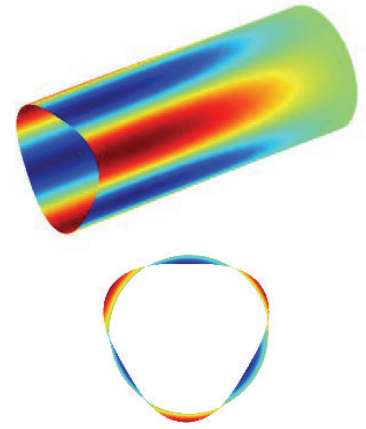

(c) 3rd mode

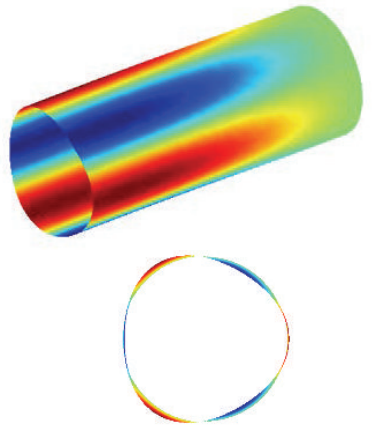

(d) 4 th mode

FIGURE 10: First four mode shapes for closed cylindrical shells with one boundary end clamped and another end elastically supported $\left(k_{\alpha L}^{u}=\right.$ $k_{\alpha L}^{v}=K_{\alpha L}^{w}=0$ and $\left.k_{\alpha L}^{w}=0.01 G\right)$.

The other parameters used in the calculations are $m=$ $1 \sim 2$ and $n=0 \sim 5$, in which $m$ and $n$ are the axial and circumferential wavenumbers. The comparisons of the first few natural frequencies with [11] are listed in Table 15. Good agreement can be seen between the present solutions and the results in [11].

In order to further validate the accuracy of the present formulation applied to cylindrical shell, a more complicated case in which the shell is elastically constrained at one end and the other end is clamped is carried out in Table 16. For simplicity and convenience, the elastically supported end only has linear springs attached in the radial direction $\left(k_{\alpha L}^{u}=\right.$ $\left.k_{\alpha L}^{v}=K_{\alpha L}^{w}=0\right)$ and $k_{\alpha L}^{w}$ varies from 0 to $10^{8} G$, where $G$ represents the in-plane stiffness $E h /\left(1-\mu^{2}\right)$. Table 16 compares the first four frequencies for several spring stiffness values calculated by the present method with those in [11], and the corresponding mode shapes clamped at one end and elastically supported at the other end $\left(k_{\alpha L}^{u}=k_{\alpha L}^{v}=K_{\alpha L}^{w}=0\right.$ and $k_{\alpha L}^{w}=0.01 G$ ) are also depicted in Figure 10. Obviously, the case for $k_{\alpha L}^{w}=0$ represents C-F supported boundary conditions. An excellent agreement can be seen from the comparison, and the difference is very small. From Tables 15 and 16, the excellent accuracy and reliability of the current solution are demonstrated. Furthermore, Table 16 shows that the first four frequencies increase when the radial spring stiffness changes from 0 to $10^{6} G$, and they almost have no change after $k_{\alpha L}^{w}=10^{6} \mathrm{G}$.
As mentioned earlier, the unified method can obtain the vibration characteristics for the shell with arbitrary boundary conditions, for example, uniform boundary restraints and nonuniform boundary supports. For the sake of completeness, the comparison of first eight frequencies for a closed cylindrical shell with single point clamped-supported at each end is listed in Table 17. The material and geometrical parameters are $E=216 \mathrm{GPa}, \mu=0.3, \rho=7900 \mathrm{~kg} / \mathrm{m}^{3}$, $R=0.237 \mathrm{~m}, h=0.003 \mathrm{~m}$, and $L=1 \mathrm{~m}$. The points are located at $(0,0)$ and $\left(L_{\alpha}, 0\right)$. From the comparison with the results calculated by Chen et al. [12] in the table, an excellent agreement is observed. From Tables 15-17, the accuracy and reliability of the unified shell formulation applied to the closed cylindrical shell are validated well. Furthermore, some other numerical results for the cylindrical shell subjected to single point support with different restraint spring stiffness are presented in Table 17, which can be used for benchmarking by researchers as well as reference data for practicing engineers.

3.3.2. Conical Shells. In this subsection, studies on the free vibration of closed conical shells are carried out. The accuracy and reliability of the present method are also examined. Table 18 lists the comparison of the first several frequency parameters $\Omega=\omega R_{1} \sqrt{\rho\left(1-\mu^{2}\right) / E}$ with those solutions obtained by Liew et al. [16] using the element free $k p$-Ritz method, Shu [18] using the GDQ method, FSDT solutions 
TABLE 17: First eight frequencies (Hz) for a closed cylindrical shell with single point clamped-supported at each end.

\begin{tabular}{lcccccccr}
\hline Modes & $\mathrm{E}^{1}-\mathrm{E}^{1}$ & $\mathrm{E}^{2}-\mathrm{E}^{2}$ & $\mathrm{E}^{3}-\mathrm{E}^{3}$ & SD-SD & S-S & \multicolumn{2}{c}{$\begin{array}{c}\text { C-C } \\
\text { Ref. [12] }\end{array}$} & $\begin{array}{c}\text { ANSYS [12] } \\
\text { Present }\end{array}$ \\
\hline 1 & 0.15698 & 0.12164 & 0.09646 & 0.01870 & 0.01658 & 0.00 & 3.01 & 0.33651 \\
2 & 19.5430 & 19.8210 & 19.4759 & 0.09810 & 19.8994 & 18.84 & 18.83 \\
3 & 21.2045 & 21.4837 & 20.8517 & 20.6303 & 20.8838 & 20.55 & 20.50 & 21.2408 \\
4 & 31.9544 & 31.6035 & 31.9604 & 21.5527 & 31.9508 & 31.63 & 34.30 & 31.9679 \\
5 & 32.8183 & 32.6079 & 32.8190 & 31.9495 & 32.8171 & 32.62 & 34.33 \\
6 & 70.1945 & 73.1681 & 67.2033 & 32.8171 & 68.2388 & 69.05 & 67.87 & 70.9657 \\
7 & 71.3327 & 75.6887 & 72.7092 & 65.8760 & 76.7896 & 73.21 & 72.94 & 75.7491 \\
8 & 97.5789 & 93.7181 & 97.4584 & 76.8374 & 97.5652 & 96.98 & 99.49 & 97.5773 \\
\hline
\end{tabular}

TABLE 18: Comparison of frequency parameters $\Omega=\omega R_{1} \sqrt{\rho\left(1-\mu^{2}\right) / E}$ for a closed conical shell with different boundary conditions $(m=1)$.

\begin{tabular}{|c|c|c|c|c|c|c|c|c|c|}
\hline \multirow{2}{*}{$\mathrm{BC}$} & \multirow{2}{*}{ Method } & \multicolumn{8}{|c|}{$n$} \\
\hline & & 0 & 1 & 2 & 3 & 4 & 5 & 6 & 7 \\
\hline \multirow{5}{*}{ SD-SD } & Ref. [16] & 0.2234 & 0.5462 & 0.6309 & 0.5061 & 0.3941 & 0.3337 & 0.3235 & 0.3510 \\
\hline & Ref. [18] & 0.2233 & 0.5463 & 0.6310 & 0.5062 & 0.3942 & 0.3340 & 0.3239 & 0.3514 \\
\hline & Ref. [27] & 0.2230 & 0.5460 & 0.6308 & 0.5061 & 0.3941 & 0.3336 & 0.3232 & 0.3504 \\
\hline & Ref. [48] & 0.2233 & 0.5461 & 0.6308 & 0.5062 & 0.3941 & 0.3337 & 0.3233 & 0.3505 \\
\hline & Present & 0.2233 & 0.5461 & 0.6309 & 0.5062 & 0.3942 & 0.3339 & 0.3238 & 0.3515 \\
\hline \multirow{5}{*}{ SD-C } & Ref. [16] & 0.8691 & 0.8113 & 0.6610 & 0.5244 & 0.4316 & 0.3822 & 0.3732 & 0.3980 \\
\hline & Ref. [18] & 0.8700 & 0.8118 & 0.6613 & 0.5246 & 0.4319 & 0.3826 & 0.3737 & 0.3987 \\
\hline & Ref. [27] & 0.8693 & 0.8115 & 0.6611 & 0.5245 & 0.4317 & 0.3823 & 0.3731 & 0.3977 \\
\hline & Ref. [48] & 0.8694 & 0.8115 & 0.6611 & 0.5246 & 0.4318 & 0.3824 & 0.3732 & 0.3978 \\
\hline & Present & 0.8697 & 0.8117 & 0.6612 & 0.5246 & 0.4318 & 0.3822 & 0.3720 & 0.3948 \\
\hline \multirow{5}{*}{$\mathrm{C}-\mathrm{C}$} & Ref. [16] & 0.8732 & 0.8120 & 0.6696 & 0.5428 & 0.4565 & 0.4088 & 0.3961 & 0.4141 \\
\hline & Ref. [18] & 0.8732 & 0.8120 & 0.6696 & 0.5428 & 0.4566 & 0.4089 & 0.3963 & 0.4143 \\
\hline & Ref. [27] & 0.8726 & 0.8117 & 0.6694 & 0.5426 & 0.4563 & 0.4085 & 0.3956 & 0.4133 \\
\hline & Ref. [48] & 0.8726 & 0.8117 & 0.6694 & 0.5427 & 0.4564 & 0.4086 & 0.3958 & 0.4135 \\
\hline & Present & 0.8729 & 0.8119 & 0.6695 & 0.5427 & 0.4565 & 0.4088 & 0.3960 & 0.4139 \\
\hline
\end{tabular}

obtained by Qu et al. [27] employing a modified variational approach, and a unified solution method presented by $\mathrm{Su}$ et al. [48] employing a modified Fourier series for conical shells subjected to different boundary conditions (i.e., SD-SD, $\mathrm{SD}-\mathrm{C}$, and $\mathrm{C}-\mathrm{C})$. The geometric and material parameters for closed conical shells are taken to be $E=168 \mathrm{GPa}, \mu=0.3$, $\rho=5700 \mathrm{~kg} / \mathrm{m}^{3}, L \sin \varphi / R_{1}=0.5, h / R_{1}=0.01, R_{1}=1 \mathrm{~m}$, and $\varphi=45^{\circ}$, where $R_{1}$ is the radius of the larger edge. Similar to the study of closed cylindrical shell, $m$ and $n$ are employed to denote axial and circumferential wavenumbers. The wavenumbers used in the calculations are $m=1$ and $n=$ $0 \sim 7$. It is seen that the present results are in good agreement with those in literature. The slight discrepancies may be due to the different solutions methods in the four studies. From the competition studies presented in Table 18, it is concluded that the present method for closed conical shells is numerically accurate.

Since vibration results for the conical shells with arbitrary elastic boundary conditions are rare in the literature, some further numerical results for a closed conical shell subjected to different elastically supported boundary conditions and their combinations with various wavenumbers ( $n=1 \sim 7$, $m=1$ ) are given in Table 19 as the benchmarking by the researchers in the future and reference data for the engineers in practical engineering, and some selected corresponding mode shapes are also depicted in Figure 11. Furthermore, the vibration results for the closed conical shell with point supported are not yet available in the literature and this case is widely used in practicing engineering. Thus, some new results are calculated for the closed conical shells with three points supported at each end using different restraint spring stiffness in Table 20, which can be used for benchmarking by researchers as well as reference data for practicing engineers. As a special case, the locations of the three-point supports at each end of the conical shell are equally spaced here. Table 19 presents the first several frequency parameters $\Omega=$ $\omega R_{0} \sqrt{\rho\left(1-\mu^{2}\right) / E}$ for a closed conical shell with various classical, elastic boundary conditions and their combinations. Table 20 gives the first eight frequencies for the conical shell with three-point supports of different boundary spring 
TABLE 19: First several frequency parameters $\Omega=\omega R_{0} \sqrt{\rho\left(1-\mu^{2}\right) / E}$ for a closed conical shell with elastic boundary conditions $(m=1)$.

\begin{tabular}{|c|c|c|c|c|c|c|c|}
\hline \multirow{2}{*}{$\mathrm{BC}$} & \multicolumn{7}{|c|}{$n$} \\
\hline & 1 & 2 & 3 & 4 & 5 & 6 & 7 \\
\hline$S D-E^{1}$ & 0.28933 & 0.20624 & 0.12470 & 0.08195 & 0.06202 & 0.05701 & 0.06119 \\
\hline$S D-E^{2}$ & 0.16324 & 0.12524 & 0.10755 & 0.09154 & 0.07779 & 0.07110 & 0.07249 \\
\hline$S D-E^{3}$ & 0.32825 & 0.21771 & 0.14872 & 0.10699 & 0.08322 & 0.07325 & 0.07373 \\
\hline$C-E^{1}$ & 0.30218 & 0.22873 & 0.14741 & 0.09222 & 0.07081 & 0.06334 & 0.06230 \\
\hline$C-E^{2}$ & 0.16674 & 0.12956 & 0.12594 & 0.09838 & 0.08447 & 0.07970 & 0.07500 \\
\hline$C-E^{3}$ & 0.33006 & 0.22365 & 0.15696 & 0.11607 & 0.09149 & 0.07906 & 0.07638 \\
\hline$E^{1}-E^{1}$ & 0.29558 & 0.20694 & 0.12597 & 0.08352 & 0.06367 & 0.05809 & 0.06180 \\
\hline$E^{2}-E^{2}$ & 0.14241 & 0.12291 & 0.10945 & 0.09596 & 0.08341 & 0.07593 & 0.07507 \\
\hline$E^{3}-E^{3}$ & 0.33004 & 0.22355 & 0.15677 & 0.11584 & 0.09126 & 0.07888 & 0.07627 \\
\hline
\end{tabular}

TABLE 20: First eight frequencies $(\mathrm{Hz})$ for a closed conical shell with three points supported at each end for different boundary spring restraints.

\begin{tabular}{|c|c|c|c|c|c|c|}
\hline \multirow{2}{*}{ Modes } & \multicolumn{6}{|c|}{$\mathrm{BC}$} \\
\hline & $E^{1}-E^{1}$ & $E^{2}-E^{2}$ & $E^{3}-E^{3}$ & SD-SD & S-S & $\mathrm{C}-\mathrm{C}$ \\
\hline 1 & 5.50848 & 5.46466 & 5.49980 & 2.67591 & 5.49922 & 5.50128 \\
\hline 2 & 7.93799 & 7.99347 & 7.99228 & 5.49943 & 7.82854 & 8.01669 \\
\hline 3 & 8.08121 & 8.29333 & 8.24191 & 6.10897 & 8.06805 & 8.25289 \\
\hline 4 & 10.9531 & 10.8214 & 10.9137 & 6.55139 & 10.8263 & 10.9334 \\
\hline 5 & 11.0659 & 11.0870 & 11.0938 & 10.6319 & 11.0359 & 11.1099 \\
\hline 6 & 12.4547 & 13.5535 & 13.4218 & 10.8251 & 13.4350 & 13.7762 \\
\hline 7 & 13.8351 & 18.5555 & 18.9106 & 13.0829 & 18.8797 & 18.9523 \\
\hline 8 & 19.0665 & 21.1011 & 21.2714 & 13.2106 & 20.8085 & 21.3206 \\
\hline
\end{tabular}

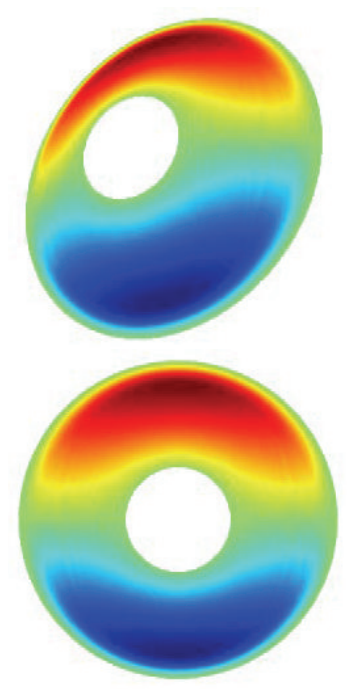

(a) $n=1$
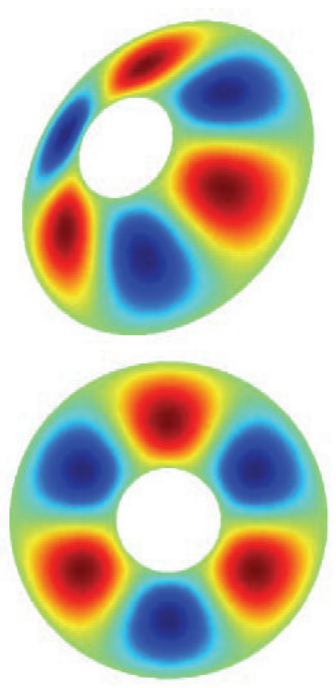

(b) $n=3$

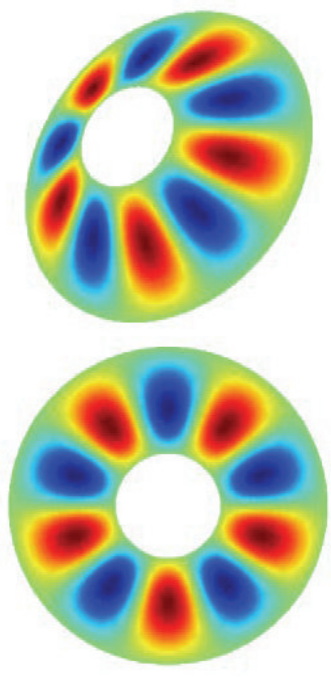

(c) $n=5$

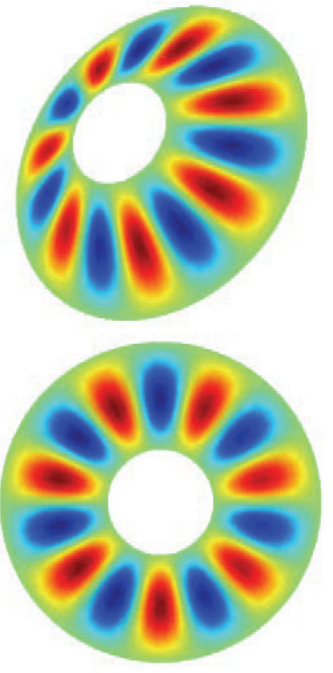

(d) $n=7$

FIGURE 11: Some selected mode shapes for closed conical shells with various wavenumbers $n$ subjected to $\mathrm{E}^{3}-\mathrm{E}^{3}$ boundary conditions $(m=1)$.

restraints. The material and geometrical parameters of the models in these two tables are employed as follows: $E=$ $210 \mathrm{GPa}, \mu=0.3, \rho=7800 \mathrm{~kg} / \mathrm{m}^{3}, R_{0}=1 \mathrm{~m}, L=3 \mathrm{~m}$, and $h=0.01 \mathrm{~m}$; in Table 19, $\varphi=45^{\circ}$; and in Table 20, $\varphi=30^{\circ}$.
3.3.3. Spherical Shells. In this subsection, the closed spherical shell with arbitrary boundary conditions, for example, classical boundary constraint, elastically supported boundary, and point supports, is considered. First, the accuracy 
TABLE 21: Comparison of the frequency parameters $\Omega=\omega R \sqrt{\rho\left(1-\mu^{2}\right) / E}$ for an annular spherical shell with different boundary conditions $(m=1)$.

\begin{tabular}{|c|c|c|c|c|c|c|c|c|c|}
\hline \multirow{2}{*}{$\mathrm{BC}$} & \multirow{2}{*}{ Method } & \multicolumn{8}{|c|}{$n$} \\
\hline & & 0 & 1 & 2 & 3 & 4 & 5 & 6 & 7 \\
\hline \multirow{3}{*}{ F-F } & Ref. [27] & 0.87054 & 0.86412 & 0.05726 & 0.15323 & 0.27900 & 0.42940 & 0.60188 & 0.79559 \\
\hline & ANSYS [27] & 0.87054 & 0.86427 & 0.05728 & 0.15330 & 0.27924 & 0.43002 & 0.60321 & 0.79810 \\
\hline & Present & 0.87140 & 0.86508 & 0.05789 & 0.15559 & 0.28434 & 0.43914 & 0.61791 & 0.82054 \\
\hline \multirow{3}{*}{$\mathrm{F}-\mathrm{C}$} & Ref. [27] & 0.81661 & 0.60880 & 0.34857 & 0.65733 & 0.96537 & 1.11557 & 1.24402 & 1.40026 \\
\hline & ANSYS [27] & 0.81659 & 0.60890 & 0.34868 & 0.65762 & 0.96592 & 1.11625 & 1.24523 & 1.40254 \\
\hline & Present & 0.81941 & 0.61013 & 0.35461 & 0.66976 & 0.97533 & 1.12665 & 1.26369 & 1.42208 \\
\hline \multirow{3}{*}{$\mathrm{C}-\mathrm{F}$} & Ref. [27] & 0.40549 & 0.15170 & 0.07636 & 0.15412 & 0.27908 & 0.42941 & 0.60189 & 0.79559 \\
\hline & ANSYS [27] & 0.40597 & 0.15184 & 0.07641 & 0.15420 & 0.27932 & 0.43003 & 0.60321 & 0.79810 \\
\hline & Present & 0.40567 & 0.12814 & 0.07251 & 0.15645 & 0.28441 & 0.43914 & 0.61787 & 0.82043 \\
\hline \multirow{3}{*}{$\mathrm{C}-\mathrm{C}$} & Ref. [27] & 0.98710 & 1.02639 & 1.00292 & 0.99803 & 1.04274 & 1.12623 & 1.24578 & 1.40056 \\
\hline & ANSYS [27] & 0.98741 & 1.02682 & 1.00340 & 0.99842 & 1.04314 & 1.12685 & 1.24697 & 1.40283 \\
\hline & Present & 0.98842 & 1.01639 & 0.97129 & 0.98775 & 1.04467 & 1.13639 & 1.26559 & 1.43364 \\
\hline
\end{tabular}

TABLE 22: First several frequency parameters $\Omega=\omega R \sqrt{\rho\left(1-\mu^{2}\right) / E}$ for a closed spherical shell with various elastic restraints $(m=1)$.

\begin{tabular}{|c|c|c|c|c|c|c|c|c|}
\hline \multirow{2}{*}{ BC } & \multicolumn{8}{|c|}{$n$} \\
\hline & 0 & 1 & 2 & 3 & 4 & 5 & 6 & 7 \\
\hline$C-E^{1}$ & 1.18313 & 1.01569 & 0.95880 & 0.97538 & 1.03337 & 1.12647 & 1.25696 & 1.42669 \\
\hline$C-E^{2}$ & 0.98843 & 0.96798 & 0.93672 & 0.96724 & 1.03005 & 1.12504 & 1.25641 & 1.42658 \\
\hline$C-E^{3}$ & 0.98274 & 1.01433 & 0.97125 & 0.98774 & 1.04461 & 1.13571 & 1.26302 & 1.42817 \\
\hline$E^{1}-E^{1}$ & 0.95591 & 1.02259 & 0.96528 & 0.97748 & 1.03389 & 1.12657 & 1.25698 & 1.42669 \\
\hline$E^{2}-E^{2}$ & 0.98843 & 0.90082 & 0.86780 & 0.93593 & 1.02066 & 1.12285 & 1.25598 & 1.42651 \\
\hline$E^{3}-E^{3}$ & 0.97787 & 1.00847 & 0.97081 & 0.98792 & 1.04458 & 1.13558 & 1.26293 & 1.42814 \\
\hline
\end{tabular}

and reliability of the unified formulation applied to closed spherical shells are verified. Table 21 compares the first several frequency parameters $\Omega=\omega R \sqrt{\rho\left(1-\mu^{2}\right) / E}$ for a spherical shell subjected to various classical boundary conditions with results obtained by $\mathrm{Qu}$ et al. [27]. The material and geometrical parameters are used as follows: $E=168 \mathrm{GPa}$, $\mu=0.3, \rho=5700 \mathrm{~kg} / \mathrm{m}^{3}, \varphi_{0}=\pi / 8, \varphi_{1}=\pi / 2, h / R=0.05$, and $R=1 \mathrm{~m}$. Similar to the investigation of cylindrical shell, $m$ and $n$ are used to express $\alpha$-axial and $\beta$-axial wavenumbers. The values used in the calculations are $m=1$ and $n=0 \sim 7$. From the competition in Table 21, an excellent agreement can be found. However, some slight discrepancies are seen in Table 21, which may be due to the different solutions methods in the three studies. From Table 21, it is concluded that the present method applied to closed spherical shells is numerically accurate.

Since vibration results for the closed spherical shells with more complex boundaries, such as elastic restraints and point supports, are not yet available in the literature, some new results are calculated for closed spherical shells, which can be used for benchmarking by researchers as well as reference data for practicing engineers. Table 22 gives the first several frequency parameters $\Omega=\omega R \sqrt{\rho\left(1-\mu^{2}\right) / E}$ with different wavenumbers ( $m=1$ and $n=0 \sim 7)$ subjected to various elastic restraints, and the several corresponding mode shapes with $\mathrm{E}^{3}-\mathrm{E}^{3}$ are depicted in Figure 12. Table 23 lists first eight frequencies for a closed spherical shell with single point classically and elastically supported at each end. For simplicity and convenience, the single point along each end is located at $(0,0)$ and $\left(L_{\alpha}, 0\right)$, respectively. The material properties and geometrical dimensions for the two tables are the same as the ones in Table 21. Unlike the vibrations for spherical shells with uniform boundary conditions, an interesting phenomenon can be found from Table 23 in which the discrepancy of the natural frequency is little among the various boundary conditions, which is maybe because some springs stiffness contributes little to the vibrations for single point support.

\section{Conclusion}

In this paper, a unified solution method for the free vibrations of the open and closed cylindrical, conical, and spherical shells with arbitrary boundary conditions, for example, classical, elastic, and point boundary supports, has been presented. The classical Flügge thin shell theory based on Kirchhoff assumptions is adopted to formulate the theoretical model. Regardless of the boundary conditions, the displacement of the thin shell is invariantly expressed as the expansion series using SGM, which is composed of a standard cosine series and auxiliary functions introduced to accelerate 
TABLE 23: First eight frequencies (Hz) for a closed spherical shell with single point classically and elastically supported at each end.

\begin{tabular}{|c|c|c|c|c|c|c|}
\hline \multirow{2}{*}{ Modes } & \multicolumn{6}{|c|}{$\mathrm{BC}$} \\
\hline & $E^{1}-E^{1}$ & $E^{2}-E^{2}$ & $E^{3}-E^{3}$ & SD-SD & S-S & $\mathrm{C}-\mathrm{C}$ \\
\hline 1 & 0.14643 & 0.14493 & 0.14529 & 0.09146 & 0.14496 & 0.14487 \\
\hline 2 & 24.2791 & 23.2846 & 23.3128 & 0.14486 & 24.7998 & 23.2793 \\
\hline 3 & 42.0388 & 42.0100 & 42.0387 & 20.9759 & 42.0388 & 42.0388 \\
\hline 4 & 53.2235 & 47.4842 & 47.1233 & 42.0388 & 60.6122 & 47.6368 \\
\hline 5 & 94.8830 & 90.2265 & 90.2590 & 54.9703 & 115.480 & 90.2196 \\
\hline 6 & 116.457 & 115.596 & 116.457 & 90.4786 & 116.457 & 116.457 \\
\hline 7 & 147.464 & 145.630 & 147.464 & 116.457 & 147.464 & 147.464 \\
\hline 8 & 185.994 & 183.715 & 182.568 & 147.464 & 193.759 & 183.733 \\
\hline
\end{tabular}

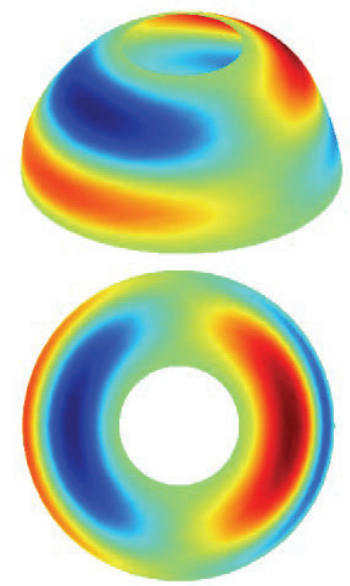

(a) $n=1$

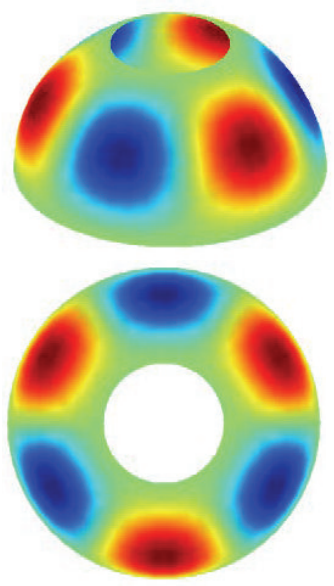

(b) $n=3$

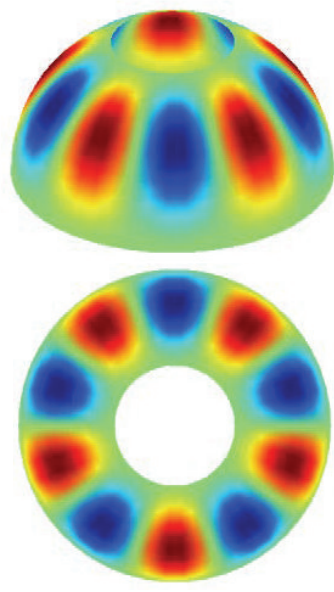

(c) $n=5$

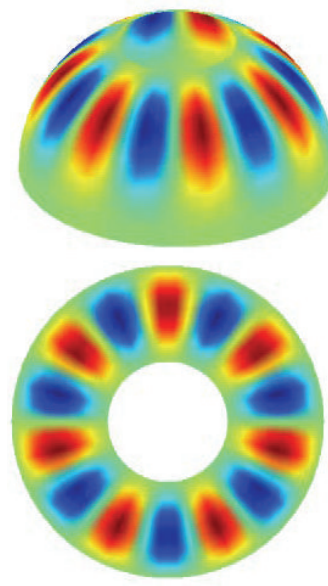

(d) $n=7$

FIGURE 12: Several selected mode shapes for closed spherical shells with different wavenumbers subjected to $\mathrm{E}^{3}$ - $\mathrm{E}^{3}(m=1)$.

the convergence of the expansion series and eliminate all the potential discontinuities at the boundary. The boundary conditions can be achieved by the artificial spring technique and different boundary conditions can be simulated conveniently by just varying the stiffness of corresponding boundary springs. The unknown expansion coefficients are solved by using the Rayleigh-Ritz method. In comparison with most existing techniques, the present method does not require any inconvenient formulation or procedural modifications to accommodate different boundary conditions or geometrical shapes for thin shells. The excellent accuracy and reliability of the proposed method have been adequately confirmed by comparing the present results with those available in literature and FEM. Based on this, a number of examples have been carried out to illustrate the influences on the vibration behavior of the geometrical dimensions and the boundary restraint parameters of open and closed shells. Moreover, some new results for the shell with various geometrical dimensions and elastic boundary conditions are presented, which can serve as a benchmark solution for other computational techniques in the future research. It should be pointed out that this solution method can be readily extended to the composite laminated and functionally graded shells with varying distributions of geometrical dimensions. Furthermore this method can also be expected to be applicable to study vibration analysis of shell combinations.

\section{Conflict of Interests}

The authors declare that there is no conflict of interests regarding the publication of this paper.

\section{Acknowledgment}

The authors gratefully acknowledge the financial supports from the National Natural Science Foundation of China (no. 51505096 and no. 51209052).

\section{References}

[1] E. Carrera, "Theories and finite elements for multilayered, anisotropic, composite plates and shells," Archives of Computational Methods in Engineering, vol. 9, no. 2, pp. 87-140, 2002.

[2] K. M. Liew, C. W. Lim, and S. Kitipornchai, "Vibration of shallow shells: a review with bibliography," Applied Mechanics Reviews, vol. 50, no. 8, pp. 431-444, 1997. 
[3] M. S. Qatu, R. W. Sullivan, and W. Wang, "Recent research advances on the dynamic analysis of composite shells: 20002009," Composite Structures, vol. 93, no. 1, pp. 14-31, 2010.

[4] A. W. Leissa, Vibration of Shells, vol. 288, Scientific and Technical Information Office, National Aeronautics and Space Administration, Washington, DC, USA, 1973.

[5] J. N. Reddy, Mechanics of Laminated Composite Plates and Shells: Theory and Analysis, CRC Press, 2004.

[6] G. B. Warburton, "Vibration of thin cylindrical shells," Journal of Mechanical Engineering Science, vol. 7, no. 4, pp. 399-407, 1965.

[7] C. W. Bert and M. Malik, "Free vibration analysis of thin cylindrical shells by the differential quadrature method," Journal of Pressure Vessel Technology, vol. 118, no. 1, pp. 1-12, 1996.

[8] K. Y. Lam and C. T. Loy, "Effects of boundary conditions on frequencies of a multi-layered cylindrical shell," Journal of Sound and Vibration, vol. 188, no. 3, pp. 363-384, 1995.

[9] C. T. Loy and K. Y. Lam, "Vibration of thick cylindrical shells on the basis of three-dimensional theory of elasticity," Journal of Sound and Vibration, vol. 226, no. 4, pp. 719-737, 1999.

[10] M. R. Mofakhami, H. H. Toudeshky, and S. H. Hashemi, "Finite cylinder vibrations with different end boundary conditions," Journal of Sound and Vibration, vol. 297, no. 1-2, pp. 293-314, 2006.

[11] L. Dai, T. Yang, J. Du, W. L. Li, and M. J. Brennan, "An exact series solution for the vibration analysis of cylindrical shells with arbitrary boundary conditions," Applied Acoustics, vol. 74, no. 3, pp. 440-449, 2013.

[12] Y. Chen, G. Jin, and Z. Liu, "Free vibration analysis of circular cylindrical shell with non-uniform elastic boundary constraints," International Journal of Mechanical Sciences, vol. 74, pp. 120-132, 2013.

[13] S. M. R. Khalili, A. Davar, and K. Malekzadeh Fard, "Free vibration analysis of homogeneous isotropic circular cylindrical shells based on a new three-dimensional refined higher-order theory," International Journal of Mechanical Sciences, vol. 56, no. 1, pp. 1-25, 2012.

[14] G. Jin, T. Ye, Y. Chen, Z. Su, and Y. Yan, "An exact solution for the free vibration analysis of laminated composite cylindrical shells with general elastic boundary conditions," Composite Structures, vol. 106, pp. 114-127, 2013.

[15] E. Viola and F. Tornabene, "Vibration analysis of conical shell structures using GDQ method," Far East Journal of Applied Mathematics, vol. 25, no. 1, pp. 23-39, 2006.

[16] K. M. Liew, T. Y. Ng, and X. Zhao, "Free vibration analysis of conical shells via the element-free kp-Ritz method," Journal of Sound and Vibration, vol. 281, no. 3-5, pp. 627-645, 2005.

[17] X. Zhao and K. M. Liew, "Free vibration analysis of functionally graded conical shell panels by a meshless method," Composite Structures, vol. 93, no. 2, pp. 649-664, 2011.

[18] C. Shu, "An efficient approach for free vibration analysis of conical shells," International Journal of Mechanical Sciences, vol. 38, no. 8-9, pp. 935-949, 1996.

[19] F. Tornabene, "Free vibration analysis of functionally graded conical, cylindrical shell and annular plate structures with a four-parameter power-law distribution," Computer Methods in Applied Mechanics and Engineering, vol. 198, no. 37, pp. 29112935, 2009.

[20] F. Tornabene, E. Viola, and D. J. Inman, "2-D differential quadrature solution for vibration analysis of functionally graded conical, cylindrical shell and annular plate structures,"
Journal of Sound and Vibration, vol. 328, no. 3, pp. 259-290, 2009.

[21] C.-P. Wu and C.-Y. Lee, "Differential quadrature solution for the free vibration analysis of laminated conical shells with variable stiffness," International Journal of Mechanical Sciences, vol. 43, no. 8, pp. 1853-1869, 2001.

[22] F. Tornabene and E. Viola, "Vibration analysis of spherical structural elements using the GDQ method," Computers \& Mathematics with Applications, vol. 53, no. 10, pp. 1538-1560, 2007.

[23] J. Lee, "Free vibration analysis of spherical caps by the pseudospectral method," Journal of Mechanical Science and Technology, vol. 23, no. 1, pp. 221-228, 2009.

[24] B. P. Gautham and N. Ganesan, "Free vibration characteristics of isotropic and laminated orthotropic spherical caps," Journal of Sound and Vibration, vol. 204, no. 1, pp. 17-40, 1997.

[25] J.-H. Kang and A. W. Leissa, "Three-dimensional vibrations of thick spherical shell segments with variable thickness," International Journal of Solids and Structures, vol. 37, no. 35, pp. 4811-4823, 2000.

[26] Y. Qu, X. Long, S. Wu, and G. Meng, "A unified formulation for vibration analysis of composite laminated shells of revolution including shear deformation and rotary inertia," Composite Structures, vol. 98, pp. 169-191, 2013.

[27] Y. Qu, X. Long, G. Yuan, and G. Meng, "A unified formulation for vibration analysis of functionally graded shells of revolution with arbitrary boundary conditions," Composites Part B: Engineering, vol. 50, pp. 381-402, 2013.

[28] A. Selmane and A. A. Lakis, "Dynamic analysis of anisotropic open cylindrical shells," Computers \& Structures, vol. 62, no. 1, pp. 1-12, 1997.

[29] S. Kandasamy and A. V. Singh, "Free vibration analysis of skewed open circular cylindrical shells," Journal of Sound and Vibration, vol. 290, no. 3, pp. 1100-1118, 2006.

[30] X. M. Zhang, G. R. Liu, and K. Y. Lam, "Frequency analysis of cylindrical panels using a wave propagation approach," Applied Acoustics, vol. 62, no. 5, pp. 527-543, 2001.

[31] S. D. Yu, W. L. Cleghorn, and R. G. Fenton, "On the accurate analysis of free vibration of open circular cylindrical shells," Journal of Sound and Vibration, vol. 188, no. 3, pp. 315-336, 1995.

[32] N. S. Bardell, J. M. Dunsdon, and R. S. Langley, "On the free vibration of completely free, open, cylindrically curved isotropic shell panels," Journal of Sound and Vibration, vol. 207, no. 5, pp. 647-669, 1997.

[33] N. S. Bardell, J. M. Dunsdon, and R. S. Langley, "Free vibration of thin, isotropic, open, conical panels," Journal of Sound and Vibration, vol. 217, no. 2, pp. 297-320, 1998.

[34] C. W. Lim and S. Kitipornchai, "Effects of subtended and vertex angles on the free vibration of open conical shell panels: a conical co-ordinate approach," Journal of Sound and Vibration, vol. 219, no. 5, pp. 813-835, 1999.

[35] X. Zhao, Q. Li, K. M. Liew, and T. Y. Ng, “The element-free kpRitz method for free vibration analysis of conical shell panels," Journal of Sound and Vibration, vol. 295, no. 3-5, pp. 906-922, 2006.

[36] K. M. Liew, L. X. Peng, and T. Y. Ng, “Three-dimensional vibration analysis of spherical shell panels subjected to different boundary conditions," International Journal of Mechanical Sciences, vol. 44, no. 10, pp. 2103-2117, 2002.

[37] T. Ye, G. Jin, Y. Chen, and S. Shi, "A unified formulation for vibration analysis of open shells with arbitrary boundary 
conditions," International Journal of Mechanical Sciences, vol. 81, pp. 42-59, 2014.

[38] M. S. Qatu and E. Asadi, "Vibration of doubly curved shallow shells with arbitrary boundaries," Applied Acoustics, vol. 73, no. 1, pp. 21-27, 2012.

[39] S. Jiang, T. Yang, W. L. Li, and J. Du, "Vibration analysis of doubly curved shallow shells with elastic edge restraints," Journal of Vibration and Acoustics, vol. 135, no. 3, Article ID 034502, 2013.

[40] F. Tornabene, E. Viola, and N. Fantuzzi, "General higher-order equivalent single layer theory for free vibrations of doublycurved laminated composite shells and panels," Composite Structures, vol. 104, pp. 94-117, 2013.

[41] F. A. Fazzolari and E. Carrera, "Advances in the Ritz formulation for free vibration response of doubly-curved anisotropic laminated composite shallow and deep shells," Composite Structures, vol. 101, pp. 111-128, 2013.

[42] F. Tornabene, N. Fantuzzi, M. Bacciocchi, and E. Viola, "Higherorder theories for the free vibrations of doubly-curved laminated panels with curvilinear reinforcing fibers by means of a local version of the GDQ method," Composites Part B: Engineering, vol. 81, pp. 196-230, 2015.

[43] F. A. Fazzolari and E. Carrera, "Refined hierarchical kinematics quasi-3D Ritz models for free vibration analysis of doubly curved FGM shells and sandwich shells with FGM core," Journal of Sound and Vibration, vol. 333, no. 5, pp. 1485-1508, 2014.

[44] T. Ye, G. Jin, Y. Chen, X. Ma, and Z. Su, "Free vibration analysis of laminated composite shallow shells with general elastic boundaries," Composite Structures, vol. 106, pp. 470-490, 2013.

[45] N. M. Price, M. Liu, R. Eatock Taylor, and A. J. Keane, "Vibrations of cylindrical pipes and open shells," Journal of Sound and Vibration, vol. 218, no. 3, pp. 361-387, 1998.

[46] C. W. Lim and K. M. Liew, "Vibratory behaviour of shallow conical shells by a global Ritz formulation," Engineering Structures, vol. 17, no. 1, pp. 63-70, 1995.

[47] Y. K. Cheung, W. Y. Li, and L. G. Tham, "Free vibration analysis of singly curved shell by spline finite strip method," Journal of Sound and Vibration, vol. 128, no. 3, pp. 411-422, 1989.

[48] Z. Su, G. Jin, S. Shi, T. Ye, and X. Jia, "A unified solution for vibration analysis of functionally graded cylindrical, conical shells and annular plates with general boundary conditions," International Journal of Mechanical Sciences, vol. 80, pp. 62-80, 2014.

[49] D. Shi, Q. Wang, X. Shi, and F. Pang, "A series solution for the in-plane vibration analysis of orthotropic rectangular plates with non-uniform elastic boundary constraints and internal line supports," Archive of Applied Mechanics, vol. 85, no. 1, pp. 51-73, 2015.

[50] D. Shi, Q. Wang, X. Shi, and F. Pang, "Free vibration analysis of moderately thick rectangular plates with variable thickness and arbitrary boundary conditions," Shock and Vibration, vol. 2014, Article ID 572395, 25 pages, 2014.

[51] D. Shi, Q. Wang, X. Shi, and F. Pang, "An accurate solution method for the vibration analysis of Timoshenko beams with general elastic supports," Proceedings of the Institution of Mechanical Engineers Part C: Journal of Mechanical Engineering Science, 2014.

[52] X. Shi, D. Shi, W. L. Li, and Q. Wang, "A unified method for free vibration analysis of circular, annular and sector plates with arbitrary boundary conditions," Journal of Vibration and Control, 2014.
[53] T. Ye, G. Jin, and Y. Zhang, "Vibrations of composite laminated doubly-curved shells of revolution with elastic restraints including shear deformation, rotary inertia and initial curvature," Composite Structures, vol. 133, pp. 202-225, 2015.

[54] X. Xie, H. Zheng, and G. Jin, "Free vibration of four-parameter functionally graded spherical and parabolic shells of revolution with arbitrary boundary conditions," Composites Part B: Engineering, vol. 77, pp. 59-73, 2015.

[55] X. Ma, G. Jin, S. Shi, T. Ye, and Z. Liu, "An analytical method for vibration analysis of cylindrical shells coupled with annular plate under general elastic boundary and coupling conditions," Journal of Vibration and Control, 2015.

[56] G. Jin, S. Shi, Z. Su, S. Li, and Z. Liu, "A modified Fourier-Ritz approach for free vibration analysis of laminated functionally graded shallow shells with general boundary conditions," International Journal of Mechanical Sciences, vol. 93, pp. 256-269, 2015.

[57] G.-Y. Jin, H. Chen, J.-T. Du, T.-J. Yang, and W.-Y. Li, “The influence of edge restraining stiffness on the transverse vibrations of rectangular plate structures," Journal of Marine Science and Application, vol. 9, no. 4, pp. 393-402, 2010.

[58] G. Jin, Z. Su, S. Shi, T. Ye, and S. Gao, "Three-dimensional exact solution for the free vibration of arbitrarily thick functionally graded rectangular plates with general boundary conditions," Composite Structures, vol. 108, no. 1, pp. 565-577, 2014.

[59] T. Ye, G. Jin, Z. Su, and X. Jia, "A unified Chebyshev-Ritz formulation for vibration analysis of composite laminated deep open shells with arbitrary boundary conditions," Archive of Applied Mechanics, vol. 84, no. 4, pp. 441-471, 2014.

[60] G. Jin, T. Ye, X. Ma, Y. Chen, Z. Su, and X. Xie, "A unified approach for the vibration analysis of moderately thick composite laminated cylindrical shells with arbitrary boundary conditions," International Journal of Mechanical Sciences, vol. 75, pp. 357-376, 2013.

[61] Z. Su, G. Jin, S. Shi, and T. Ye, "A unified accurate solution for vibration analysis of arbitrary functionally graded spherical shell segments with general end restraints," Composite Structures, vol. 111, no. 1, pp. 271-284, 2014.

[62] G. Jin, T. Ye, X. Jia, and S. Gao, "A general Fourier solution for the vibration analysis of composite laminated structure elements of revolution with general elastic restraints," Composite Structures, vol. 109, no. 1, pp. 150-168, 2014.

[63] Z. Su, G. Jin, and T. Ye, “Three-dimensional vibration analysis of thick functionally graded conical, cylindrical shell and annular plate structures with arbitrary elastic restraints," Composite Structures, vol. 118, pp. 432-447, 2014.

[64] T. Ye, G. Jin, Z. Su, and Y. Chen, "A modified Fourier solution for vibration analysis of moderately thick laminated plates with general boundary restraints and internal line supports," International Journal of Mechanical Sciences, vol. 80, pp. 29-46, 2014.

[65] W. L. Li, "Free vibrations of beams with general boundary conditions," Journal of Sound and Vibration, vol. 237, no. 4, pp. 709-725, 2000.

[66] W. L. Li, "Vibration analysis of rectangular plates with general elastic boundary supports," Journal of Sound and Vibration, vol. 273, no. 3, pp. 619-635, 2004.

[67] S. Jiang, W. L. Li, and T. Yang, "A spectro-geometric method for the vibration analysis of built-up structures," in Proceedings of the INTER-NOISE and NOISE-CON Congress and Conference Proceedings, Institute of Noise Control Engineering, 2013. 


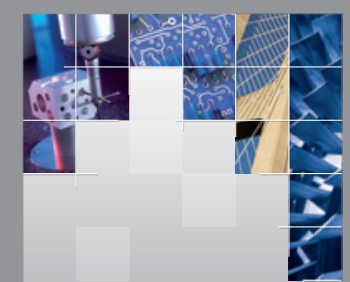

\section{Enfincering}
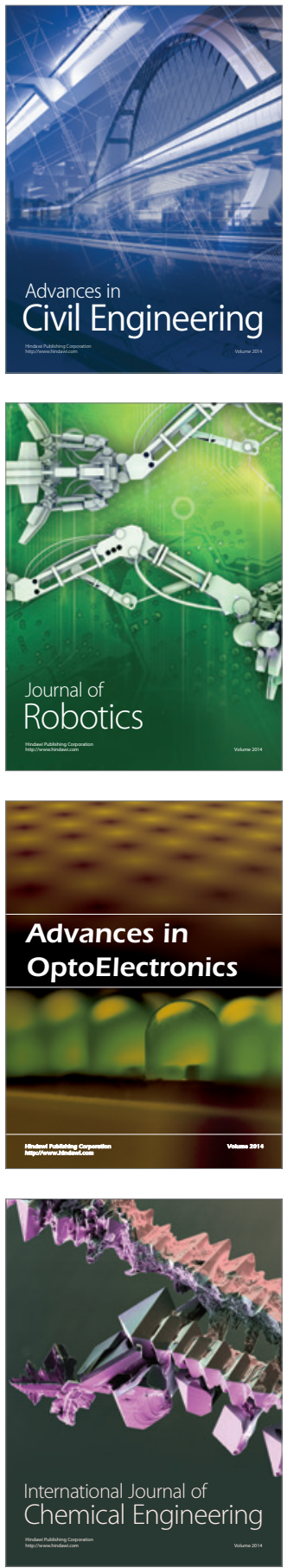

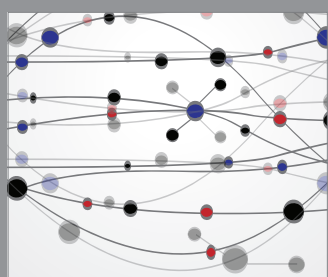

The Scientific World Journal

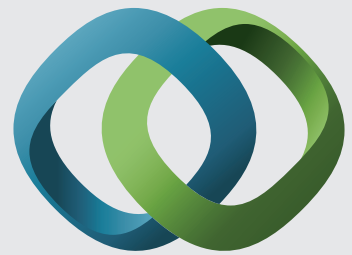

\section{Hindawi}

Submit your manuscripts at

http://www.hindawi.com
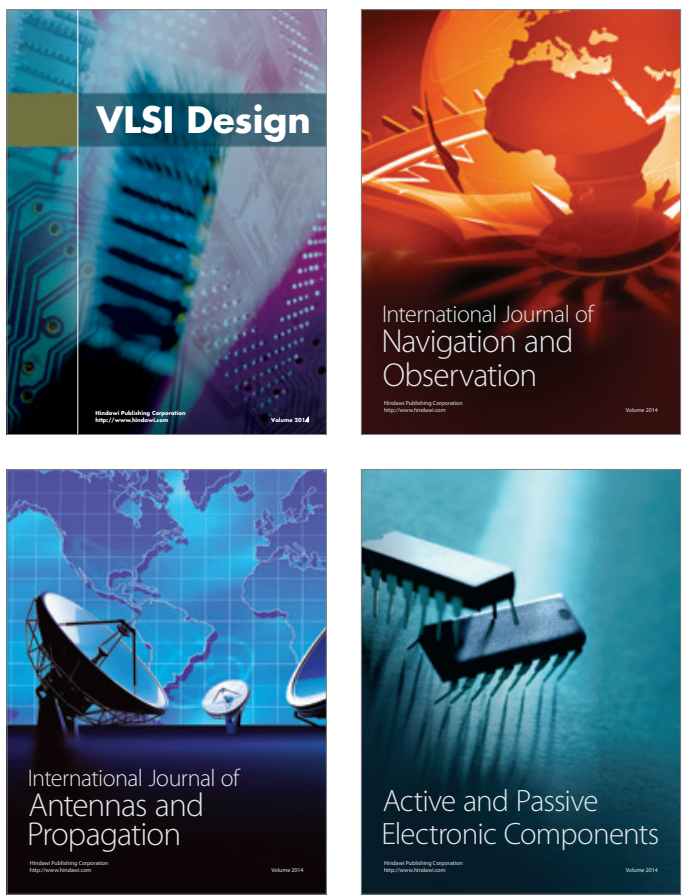
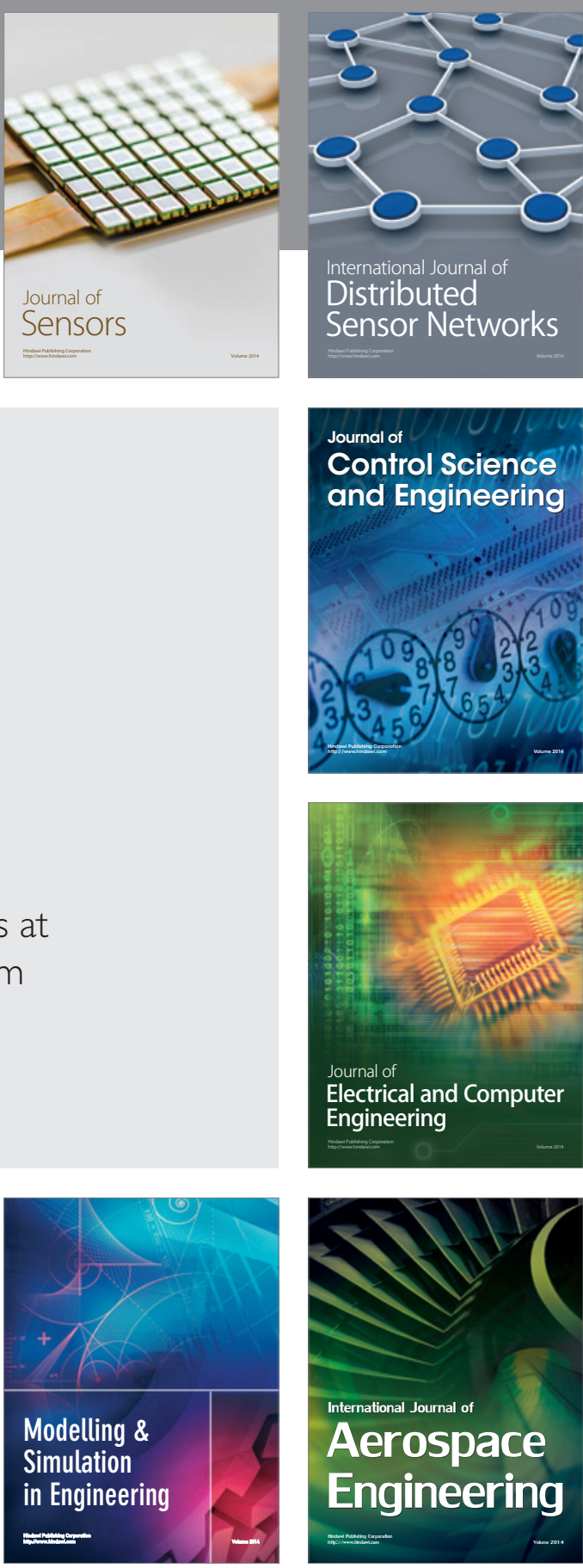

International Journal of

Distributed

Sensor Networks

Journal of

Control Science

and Engineering
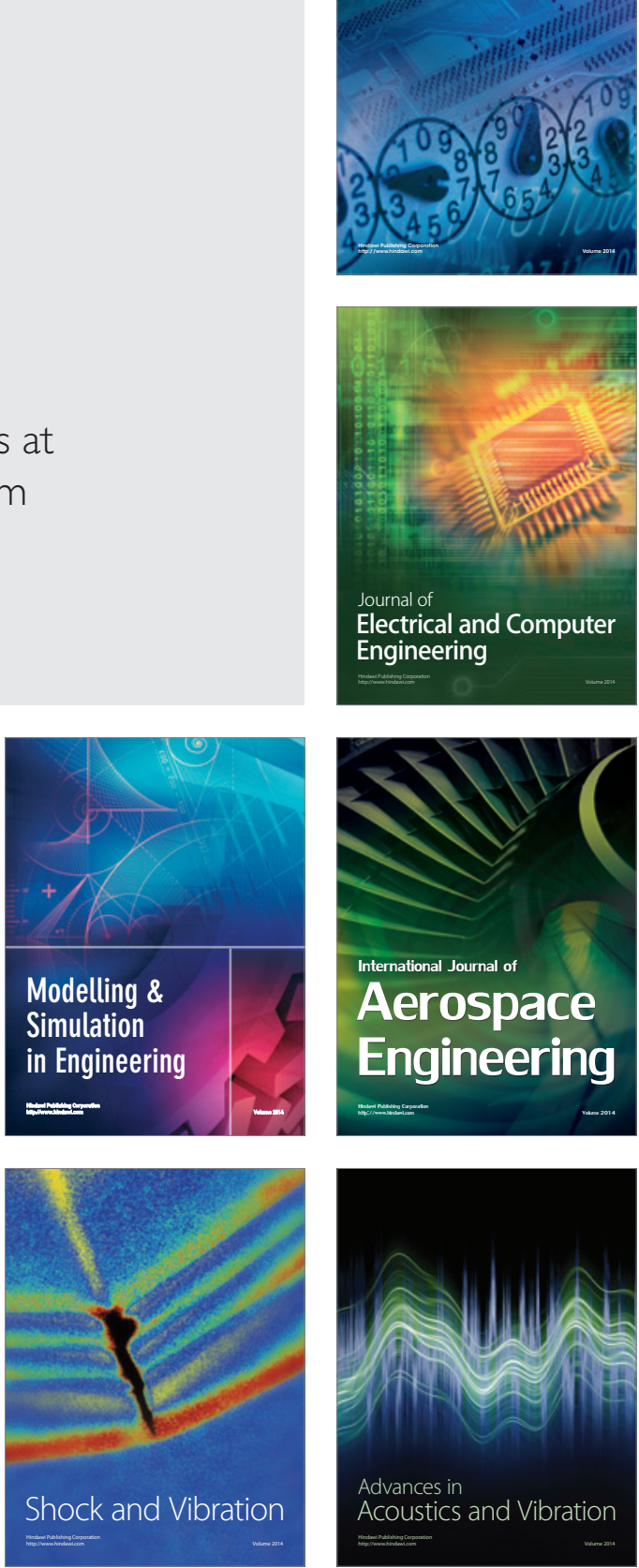